Fall 1958

\title{
1958 Miracle Yearbook
}

\section{Cedarville College}

Follow this and additional works at: https://digitalcommons.cedarville.edu/yearbooks

Part of the Higher Education Commons, Organizational Communication Commons, and the Public Relations and Advertising Commons

\section{Recommended Citation}

Cedarville College, "1958 Miracle Yearbook" (1958). Yearbooks. 39.

https://digitalcommons.cedarville.edu/yearbooks/39

This Book is brought to you for free and open access by DigitalCommons@Cedarville, a service of the Centennial Library. It has been accepted for inclusion in Yearbooks by an authorized administrator of DigitalCommons@Cedarville. For more information, please contact digitalcommons@cedarville.edu. 


$$
\begin{array}{r}
\text { M) } 3 A C(E \\
1958
\end{array}
$$





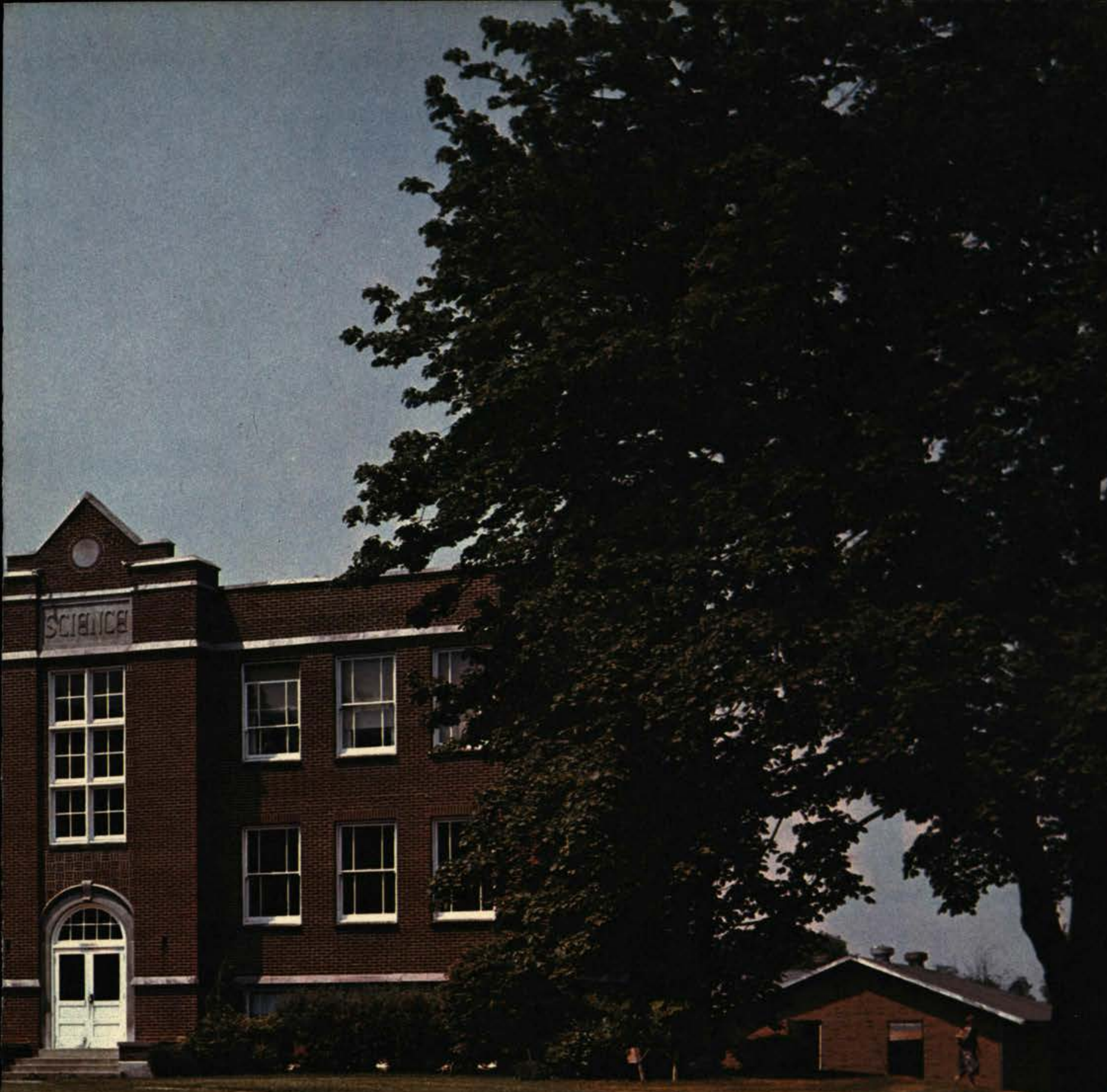


Dale SThoman 


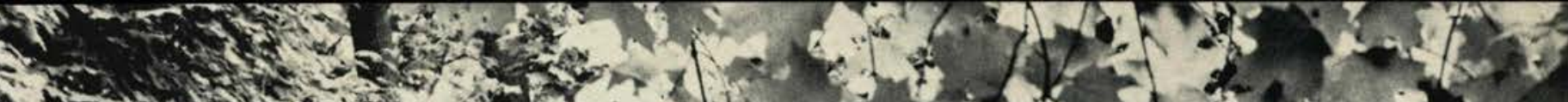

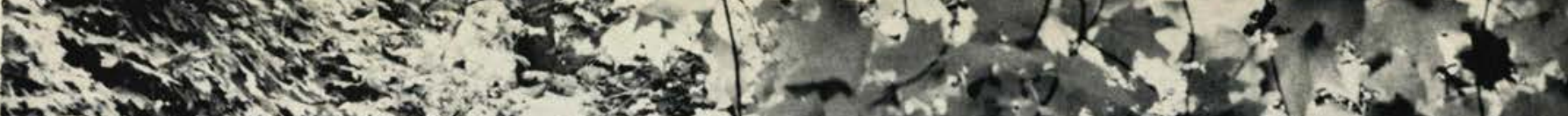
Th

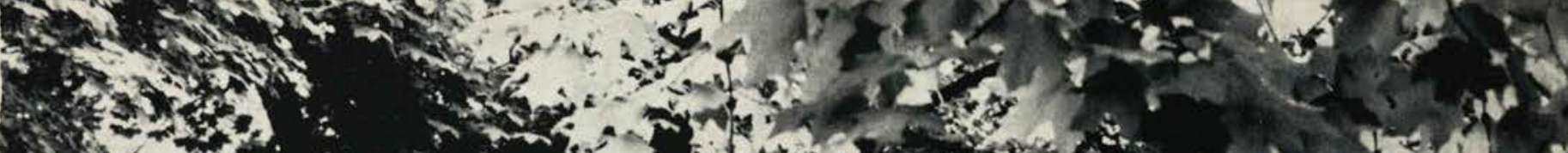

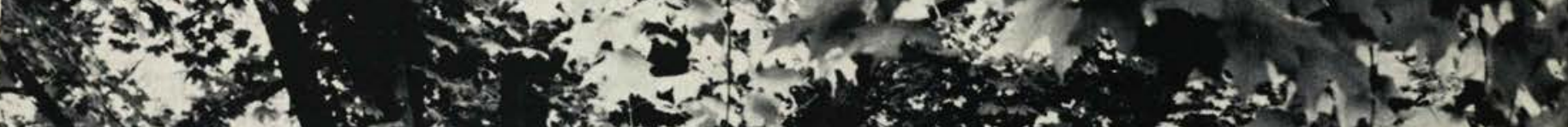

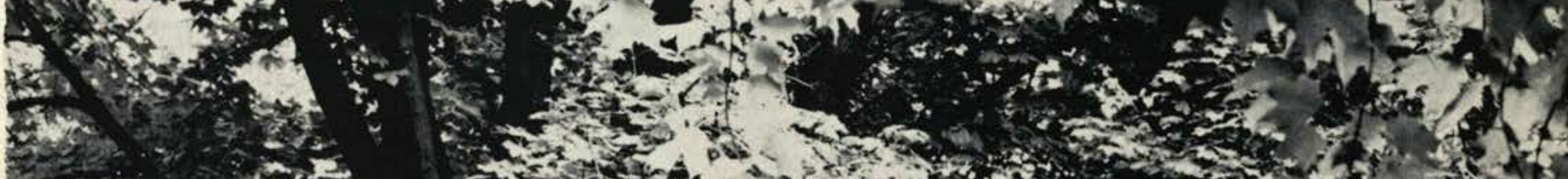

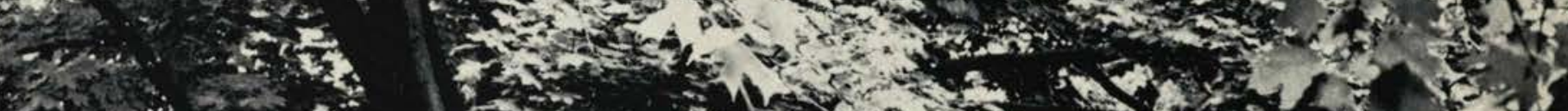

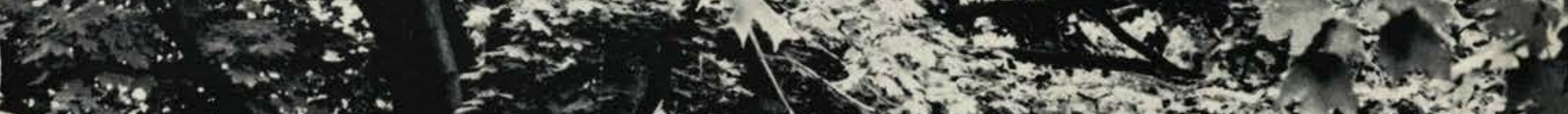

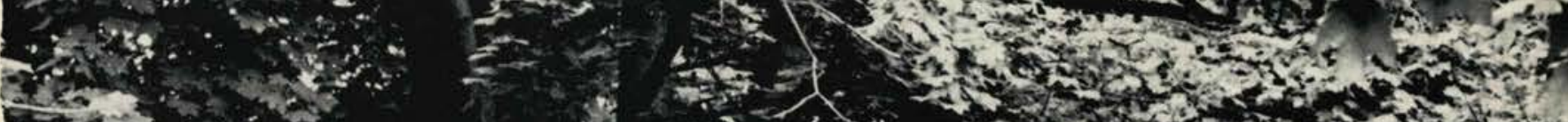

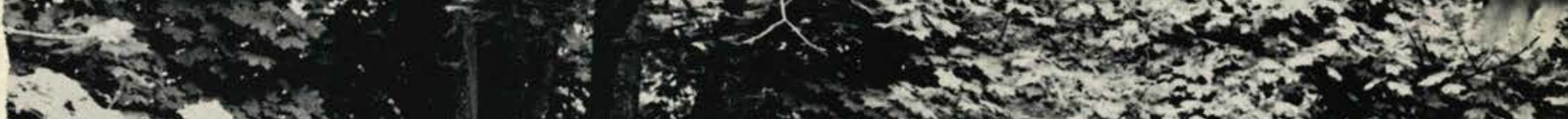

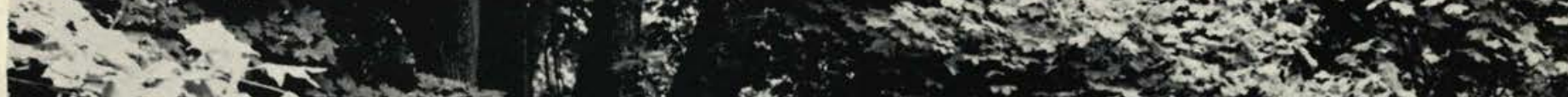
aton of

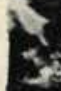

\section{<.}

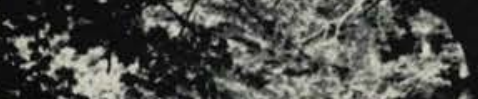

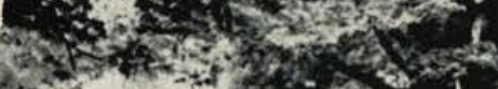

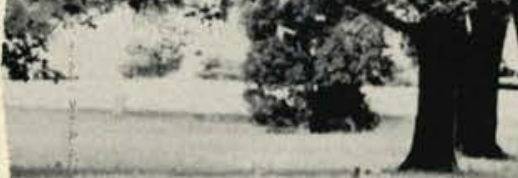

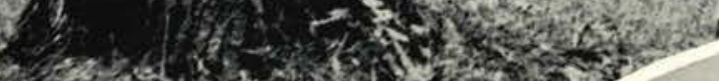

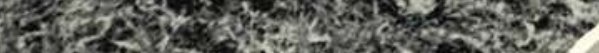

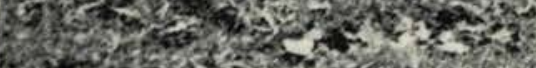

s.

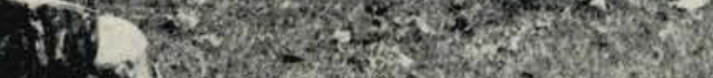

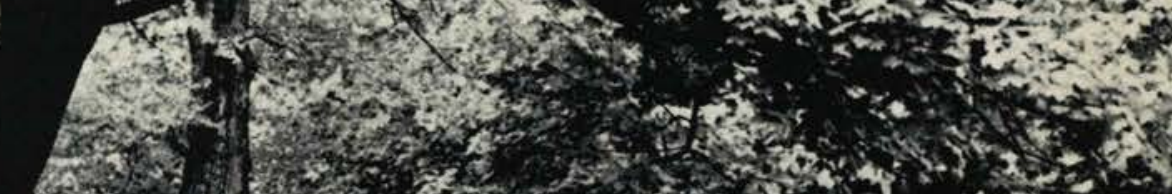

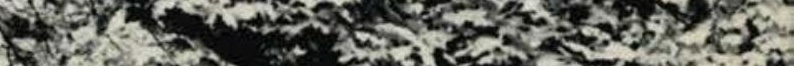

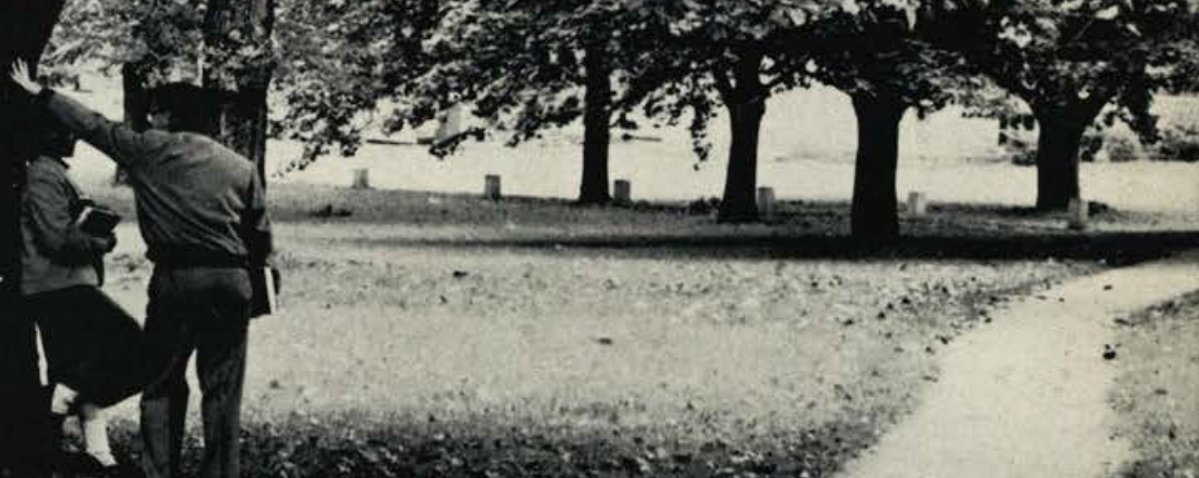

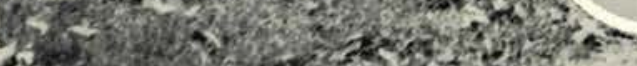
20.

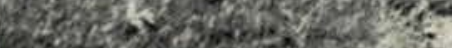

The 1958 Mirade List: 


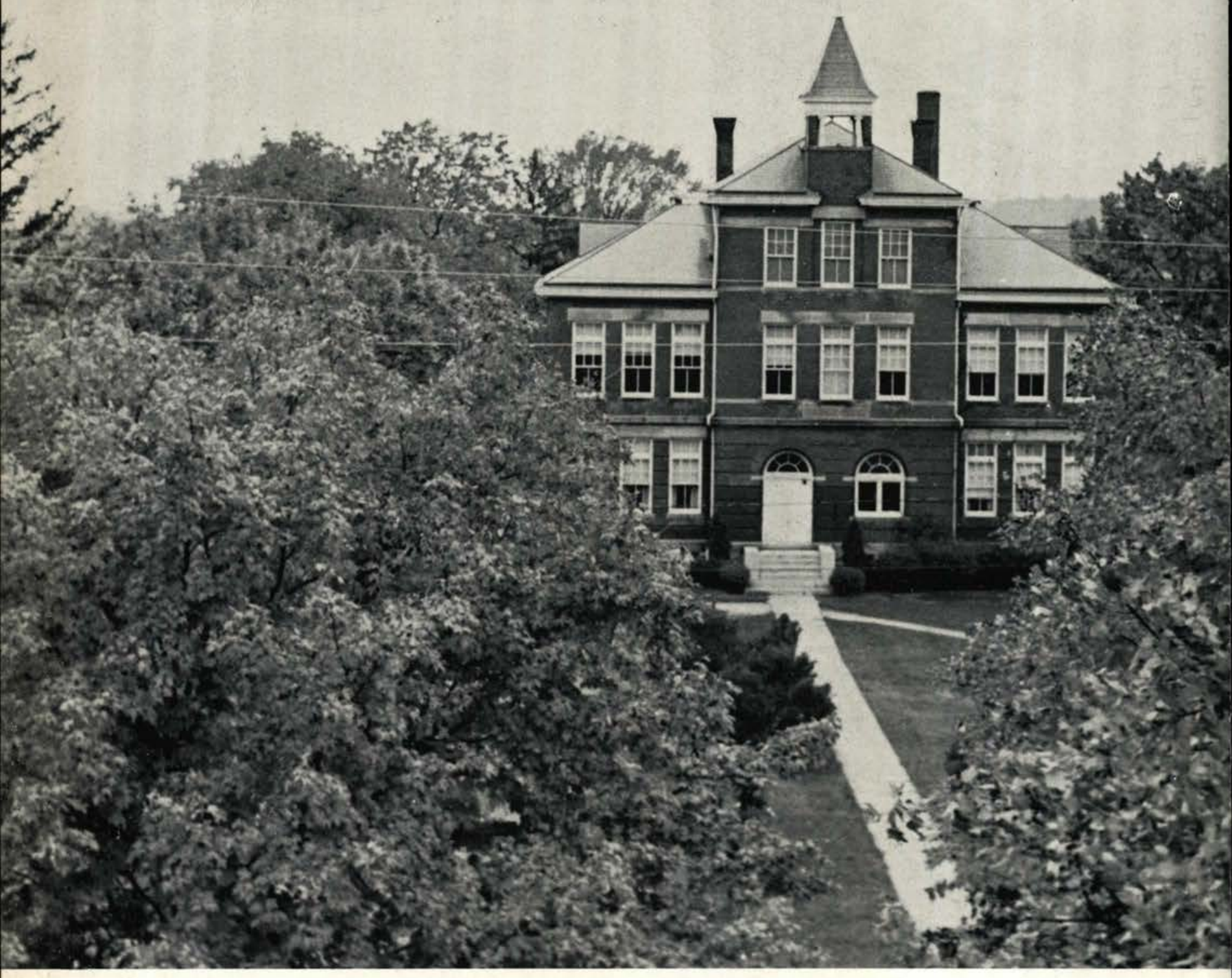

\section{Miracle Staff}

Editor

JOHN BRUMBAUGH

Assistant Editor

Art Editor RUTH YOST

Sports Editor GRACE WILLETTS

Advertising Editor
SANDRA MILLIKIN

PAUL VAN KLEEK
Typist

CAROL JOHNSON

Photographer ROBERT HOWDER

Assistant LUCY LYONS

Assistant

Advisor WARREN WOODARD MISS RUTH KANTZER 


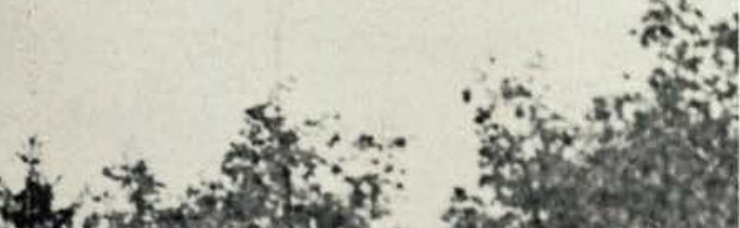

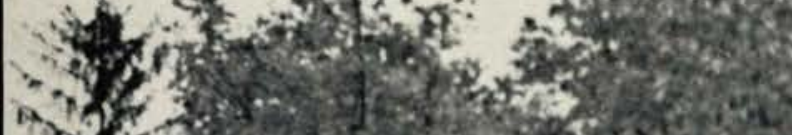

\section{Table of Contents}

Roots -

Administration

Faculty

Staff

7revele - Student Body

Bravelese - Activities

Social Events

Fruet -

Christian Service 



\section{7a Professor Bruce Turnbull}

. . who has rendered faithful service in advancing the cause of Christ at Cedarville College . . .

.. who has shown unwavering devotion to the vocation wherein God hath called him ...

. . whose desire to serve his Lord is manifested by the willingness he shows in accepting responsibilities which far exceed his line of duty . . .

. . who has given unstintingly of his time that the students of this school might be "perfect, thoroughly furnished unto all good works". . .

. . who has made untiring efforts in the improvement of the science department of Cedarville College . . .

. . we, the Junior Class, dedicate this 1958 Miracle. 



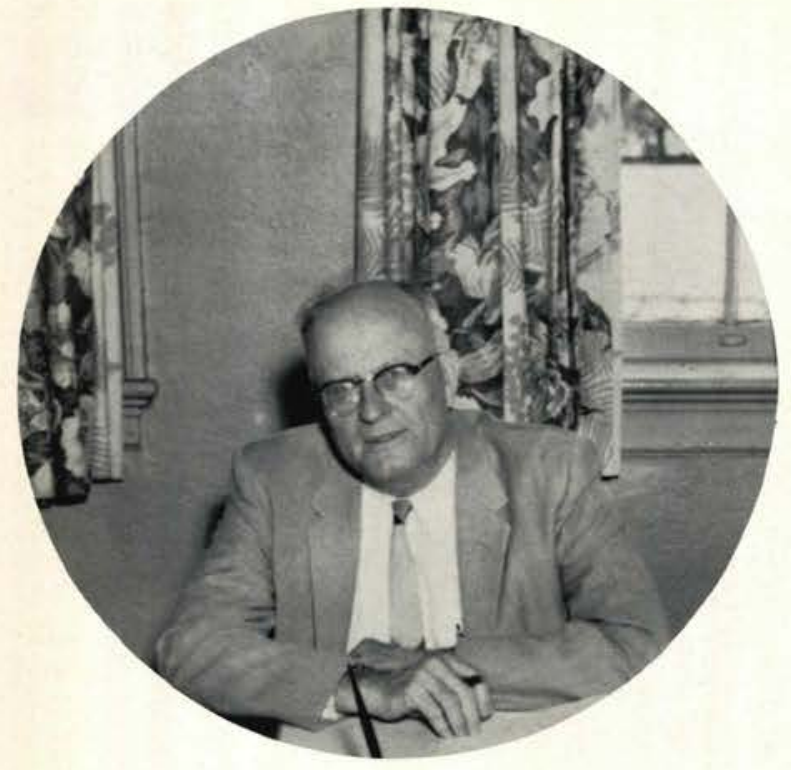

CLIFFORD MADDOX

Academic Dean

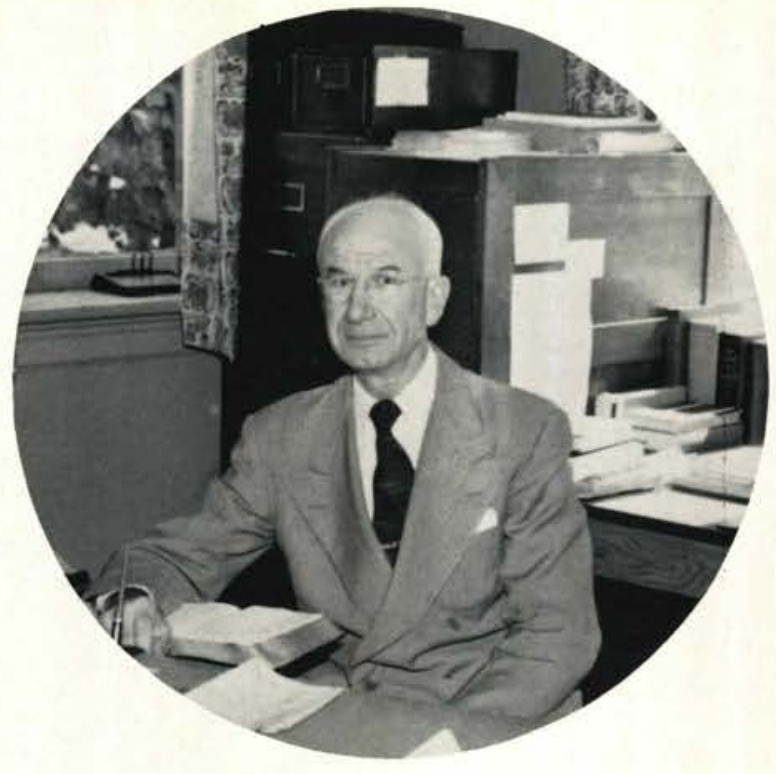

ARTHUR WILLIAMS

Dean of Students

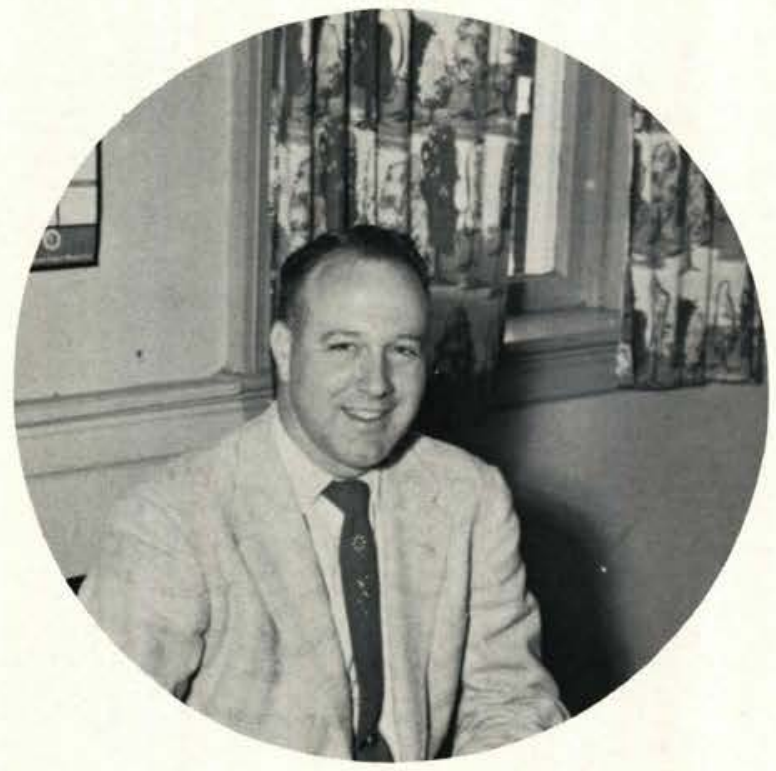

\section{ROBERT UNDERWOOD} Registrar

\section{Executive Committee}

George S. Milner, Chairman Reginald Matthews

C. C. Clawson

Andrew Tatman
James T. Jeremiah

Robert Underwood
Clifford R. Maddox

Arthur F. Williams

\section{Board of 7rustees}

Charles Barth George L. Boyd Alfred Colwell James T. Jeremiah Reginald Matthews Dale Murphy Wilber C. Rooke Gerald Smelser
George A. Bates

Norman B. Chappell

Arthur Dyke

Robert Jones

George Milner, Chairman

William Patterson

Alvin G. Ross

Eugene B. Smith

Earl V. Willetts
William Brock

C. C. Clawson

Herman Harvey

Allen Lewis

Kenneth Muck

Irving J. Reese

R. O. Sanborn

Andrew Tatman

Donald Woodby 


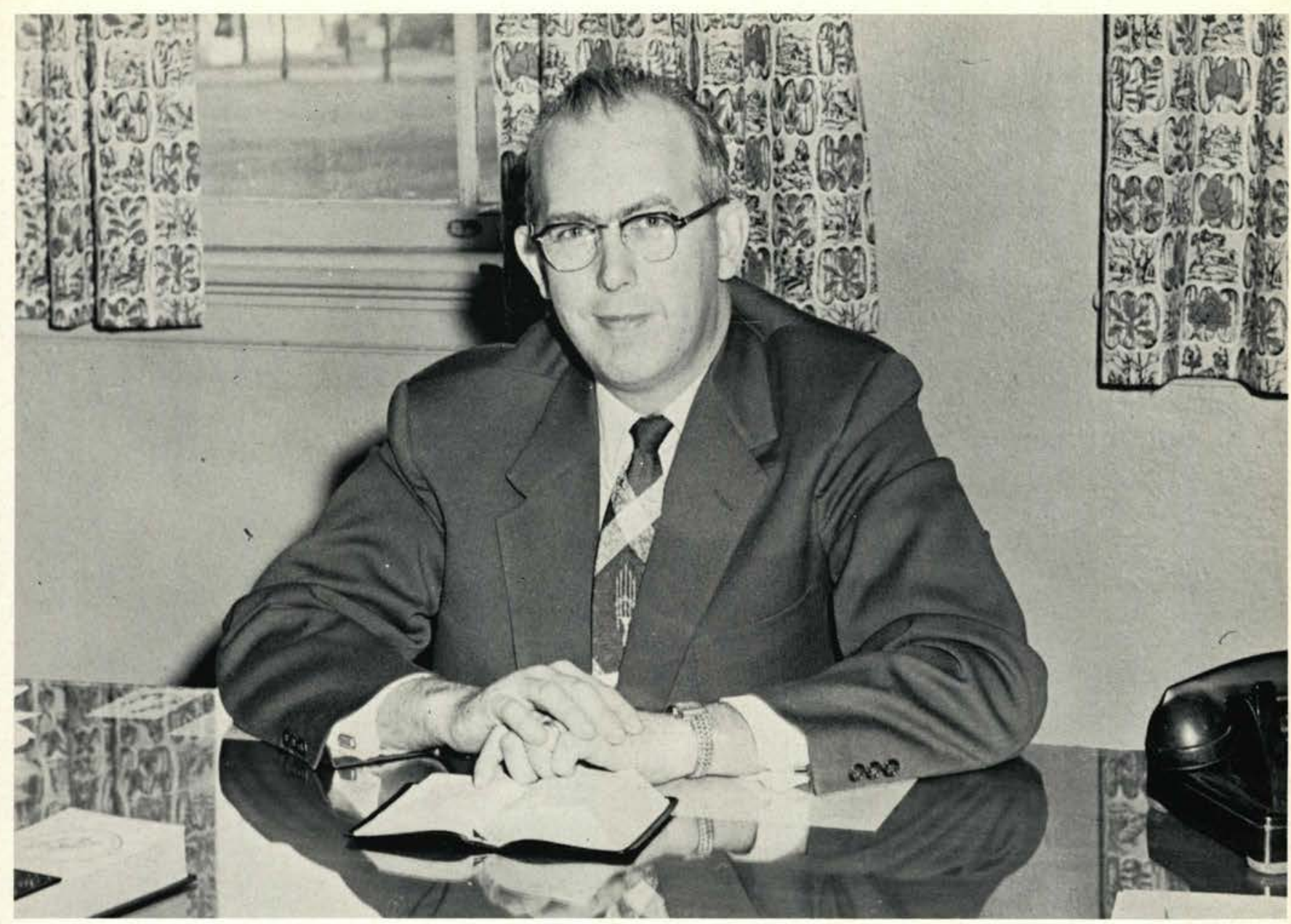

PRESIDENT JAMES T. JEREMIAH

\section{President's Message}

Christians are exhorted to "shine as lights" in a "crooked and perverted age." To do this, we must live as the sons of God, "blameless, harmless, spotless." (Phil. 2:12-14) It is our prayer that God will enable our faculty, students, and graduates to thus hold forth the word of life. This Christian life is not a passive experience but one which carries with it the greatest responsibility, since we are told to "work out your own salvation with fear and trembling." To do this we must not trust ourselves but must possess a tender conscience and always keep vigilance against temptation. We must shrink from everything which would offend or dishonor God. As the class of 1958 leaves Cedarville, we pray that God our Savior will make each member a specialist as a "light bearer" in the world, no matter what work he may be called to do. 



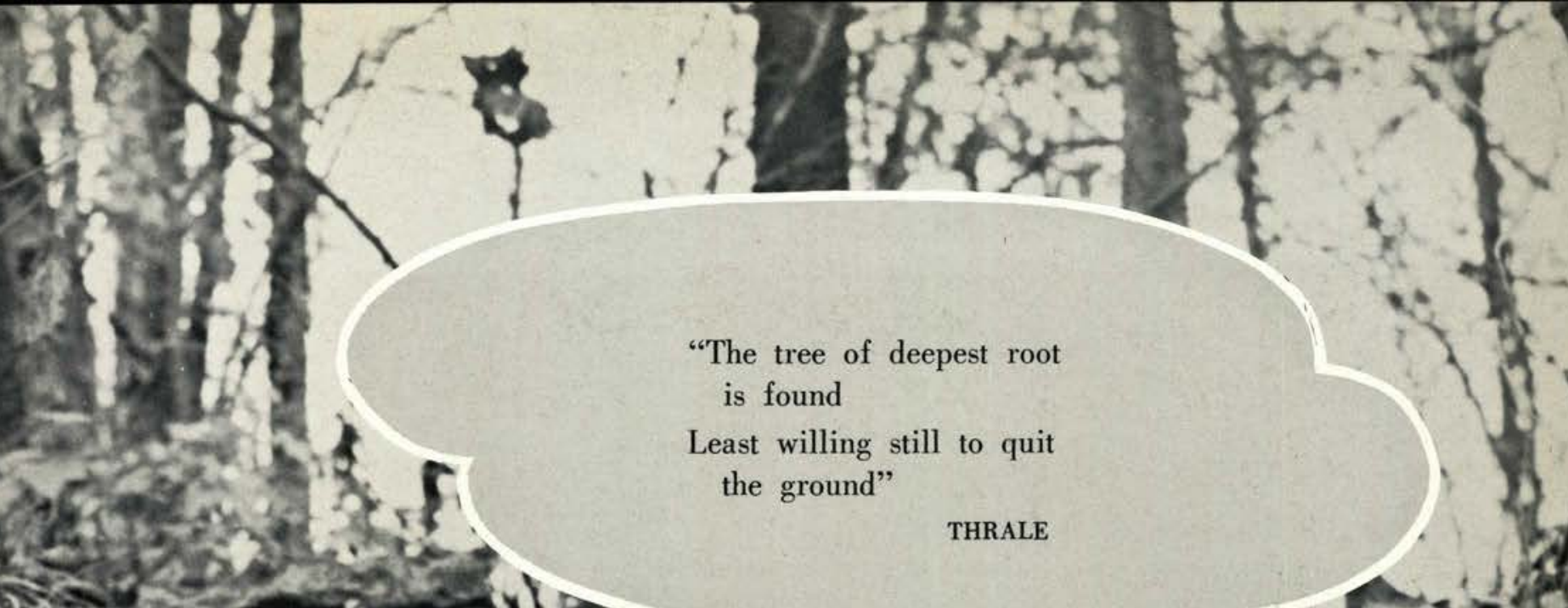

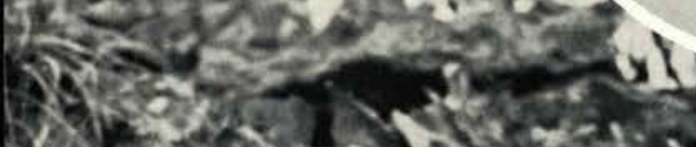

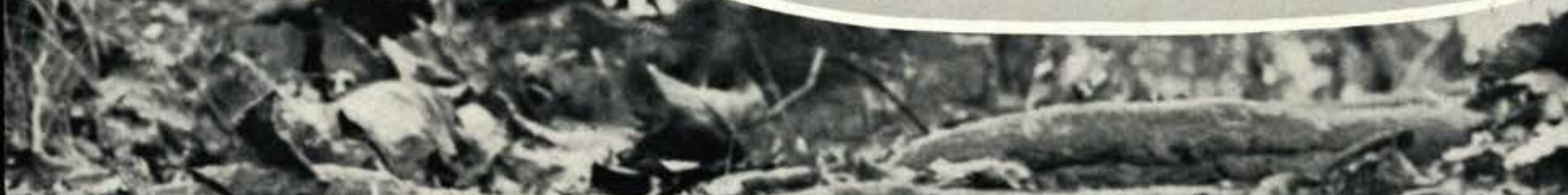

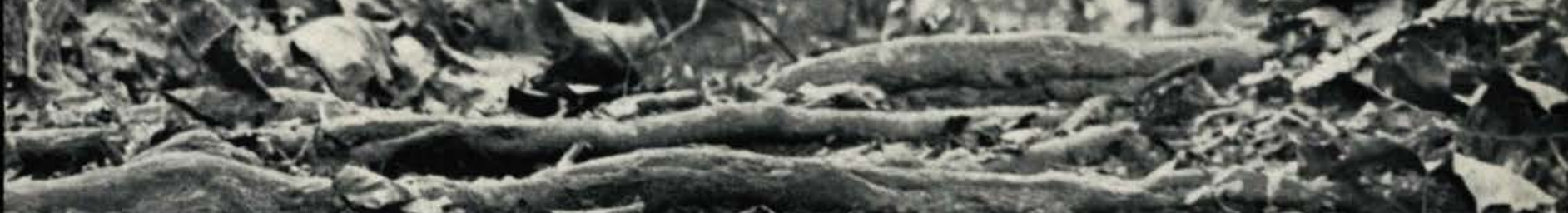

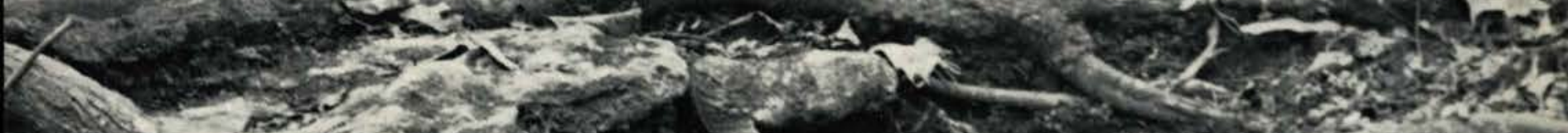

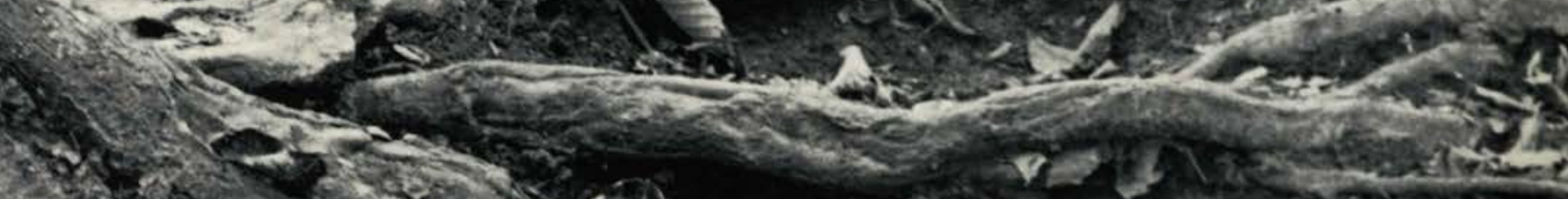
- $1.5=-1<$

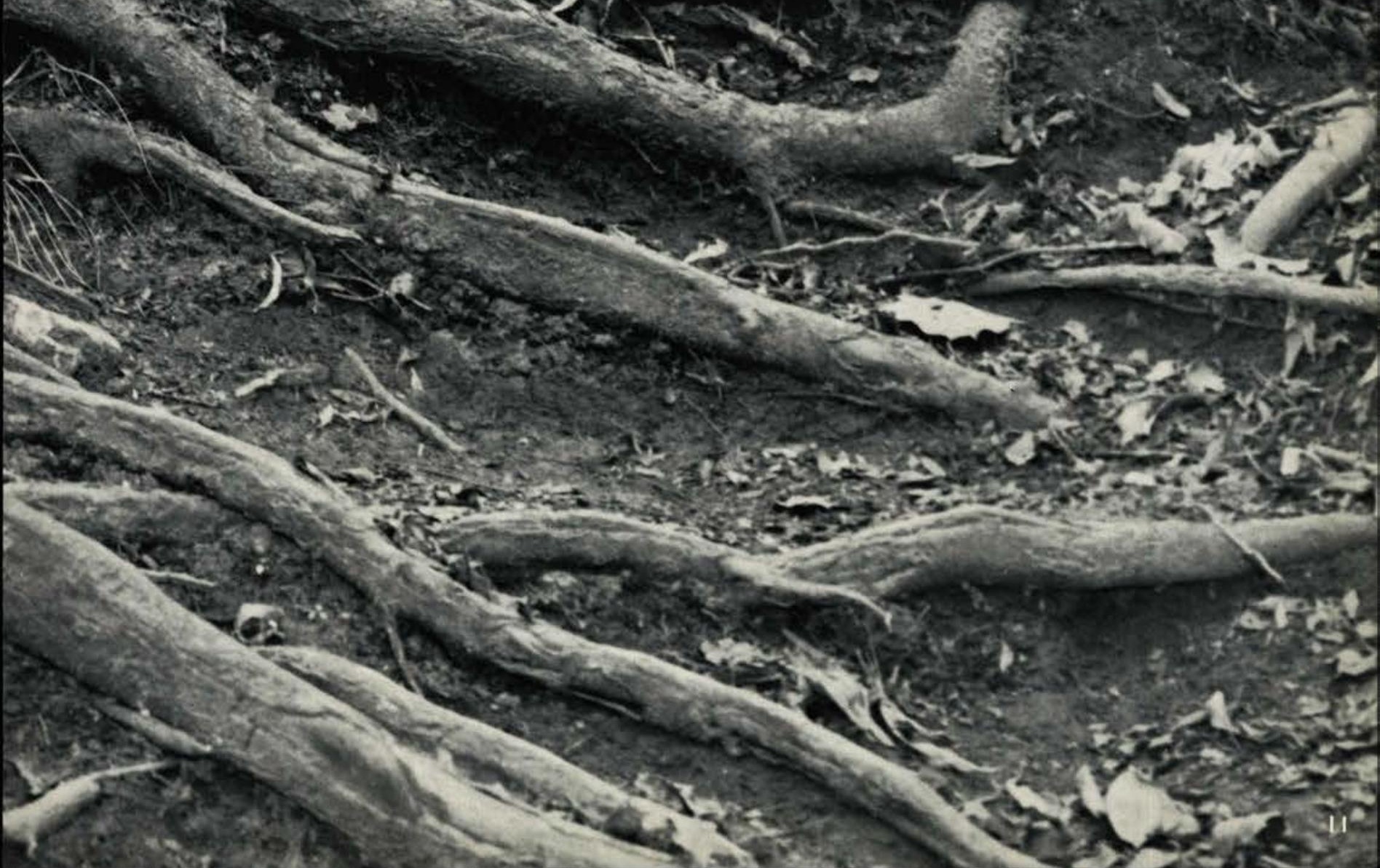




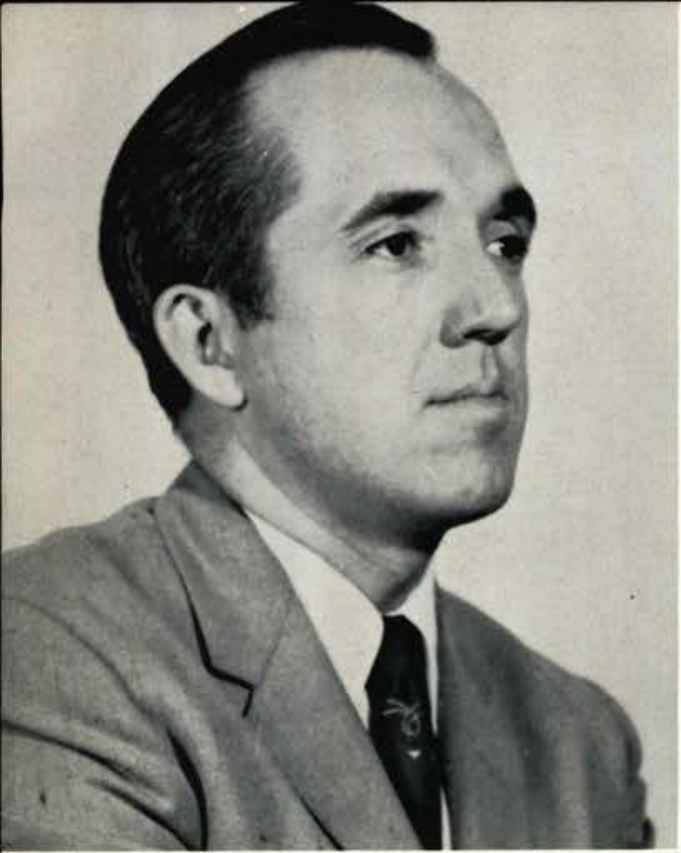

WILLIAM AMBROSE, M.A.

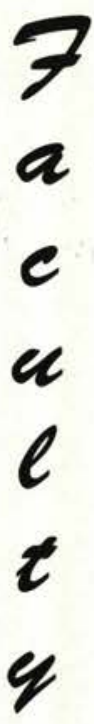

REV. GLENN GREENWOOD

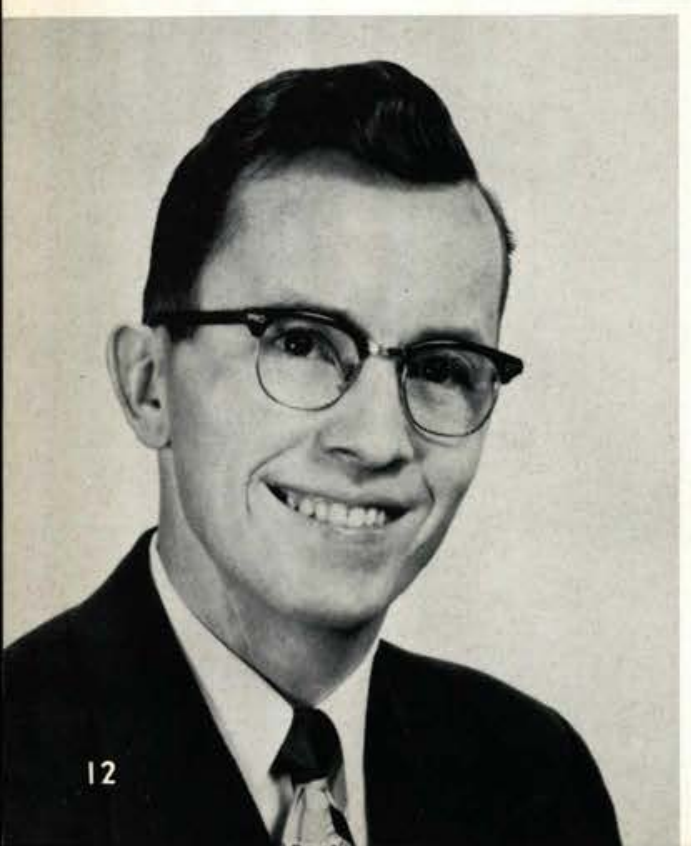

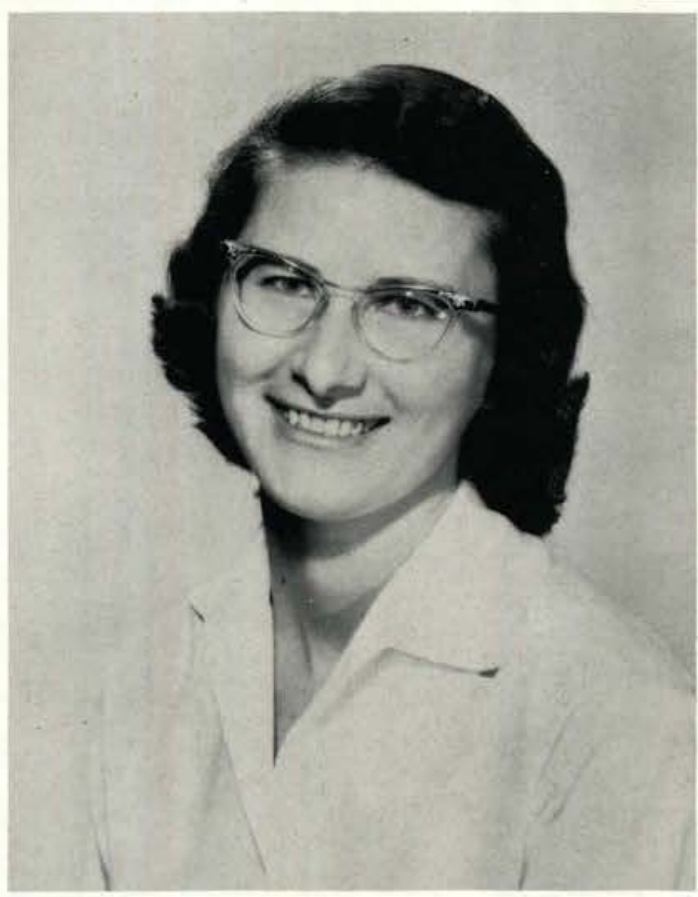

SHERWIN BOWSER, B.S.

RUTH KANTZER, B.A.

JEAN FISHER, B.R.E. 


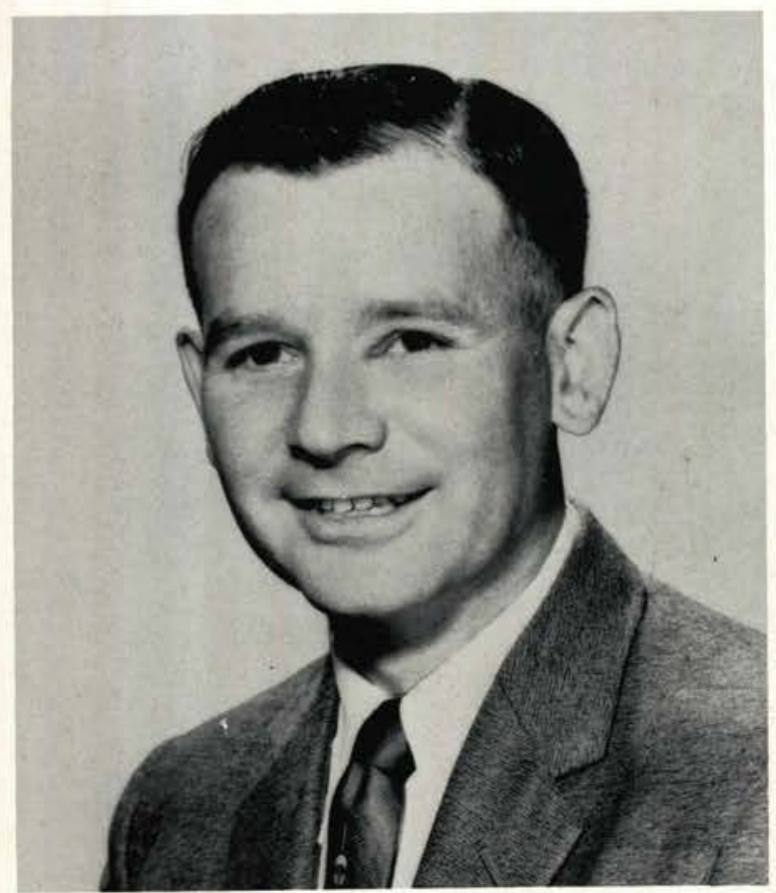

WARREN WEBBER, M.Mus.Ed.

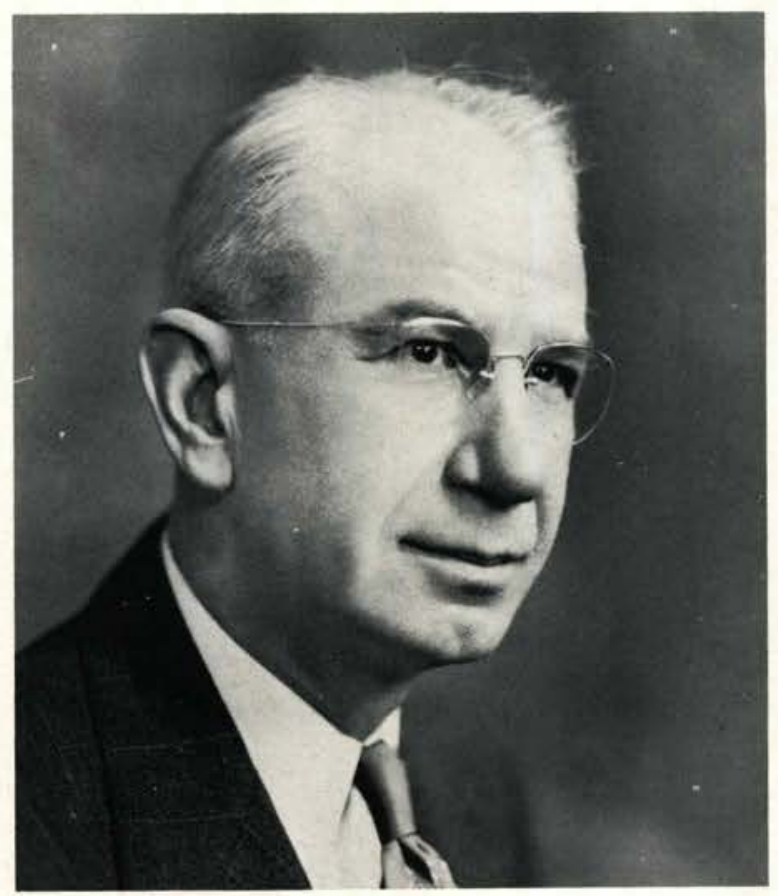

ARTHUR WILLIAMS, B.A., D.D.

\section{Faculty}

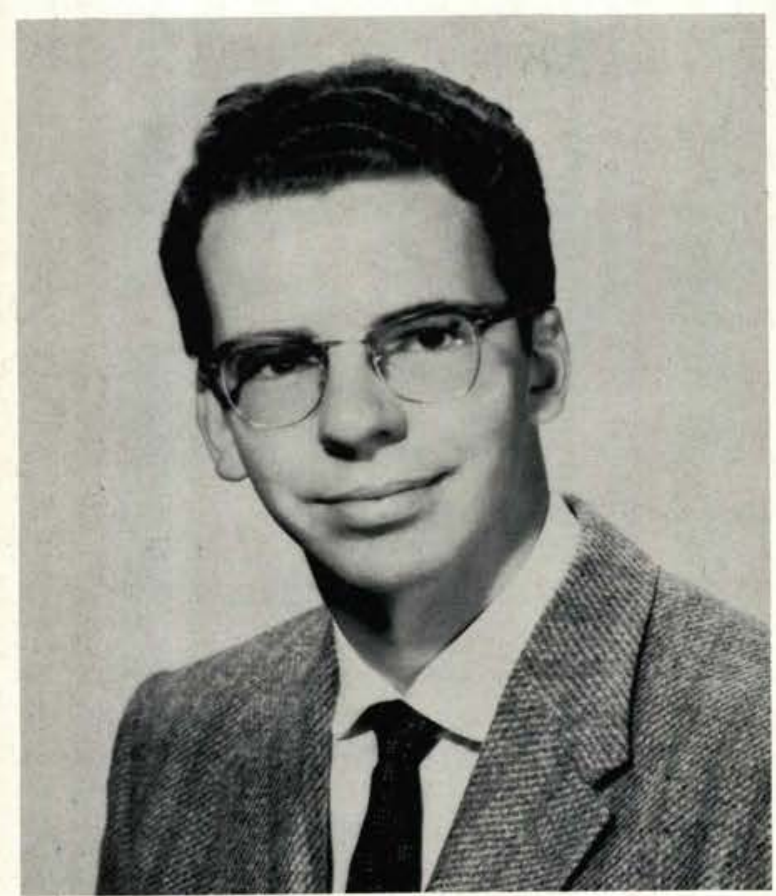

GLENN WITTIG, B.A.

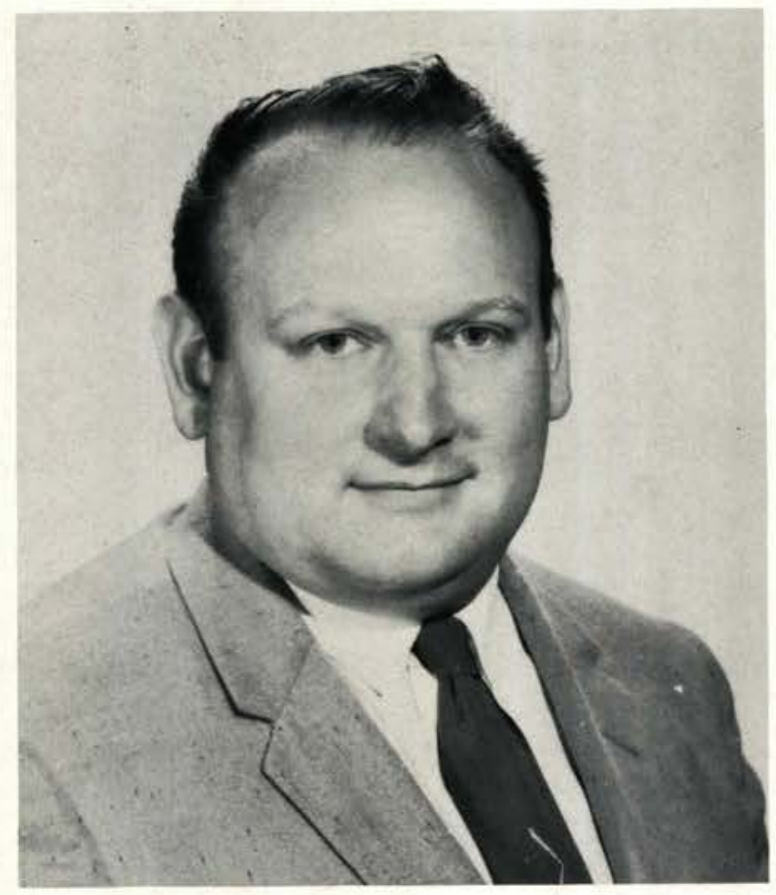

G. PAUL WYLAND, M.A. 


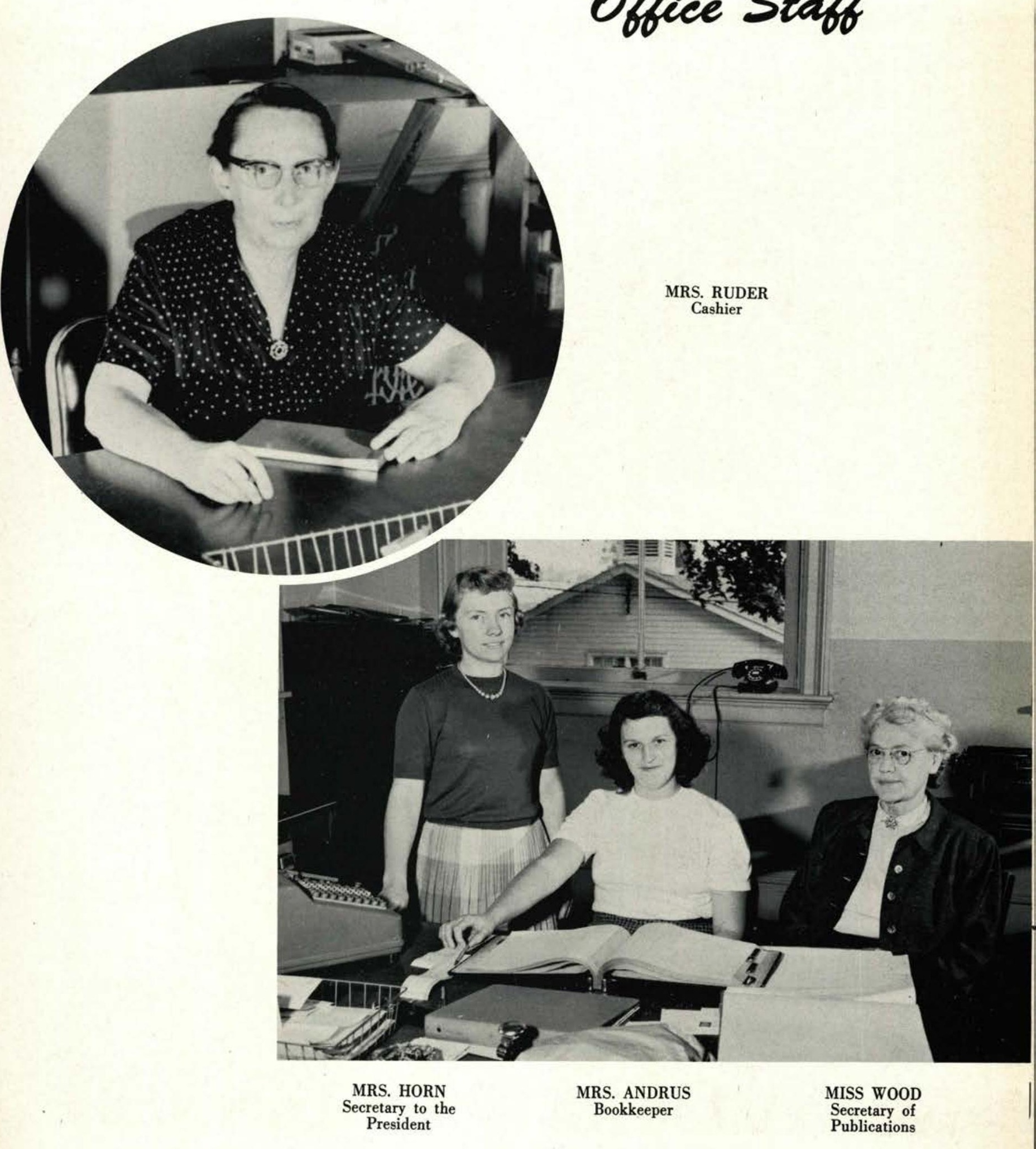

Office Staff

S. RUDER

Cashier 


\section{Library}

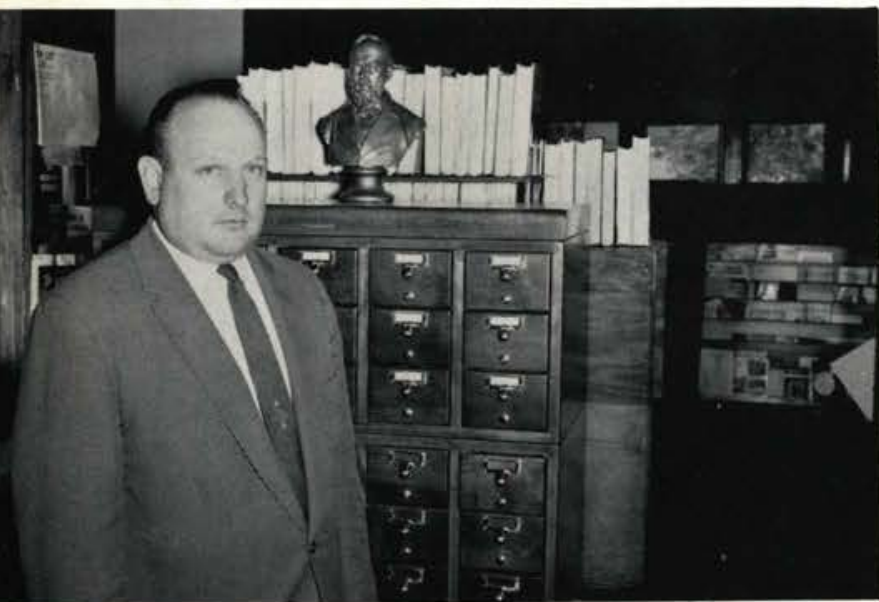

MR. WYLAND Librarian

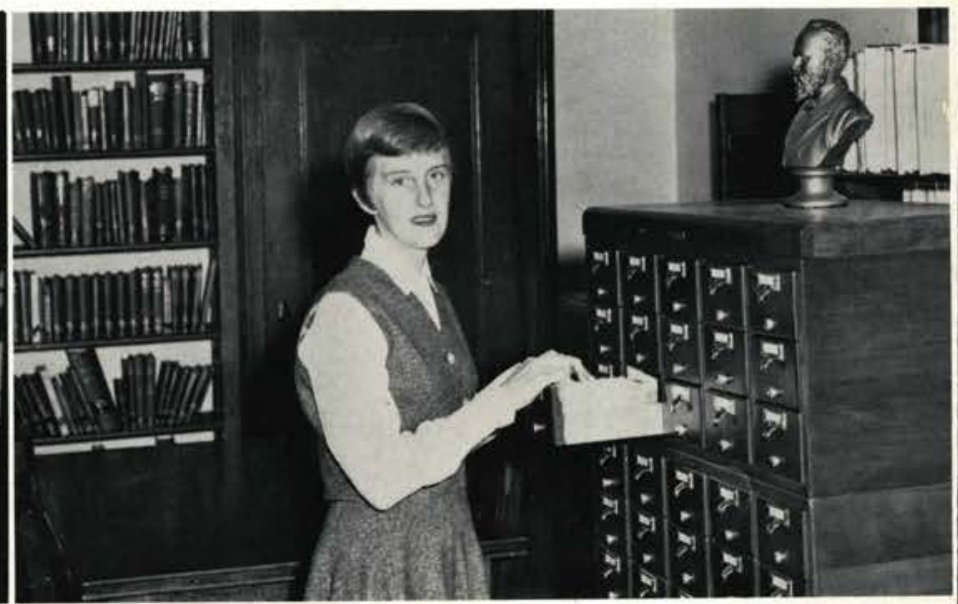

MISS R. CARZOO

Branch Librarian

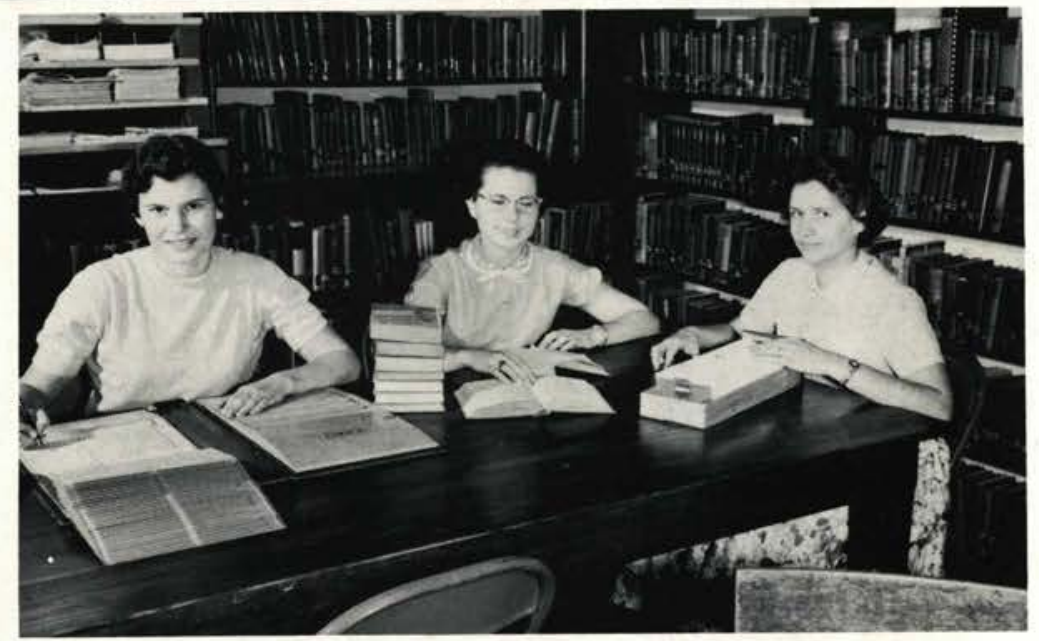

Assistants: ALBERTA CARR, HELEN STEVENS, ALBERTA CHAFFE

\section{Launge}

BERNICE MICK, Book Store Manager

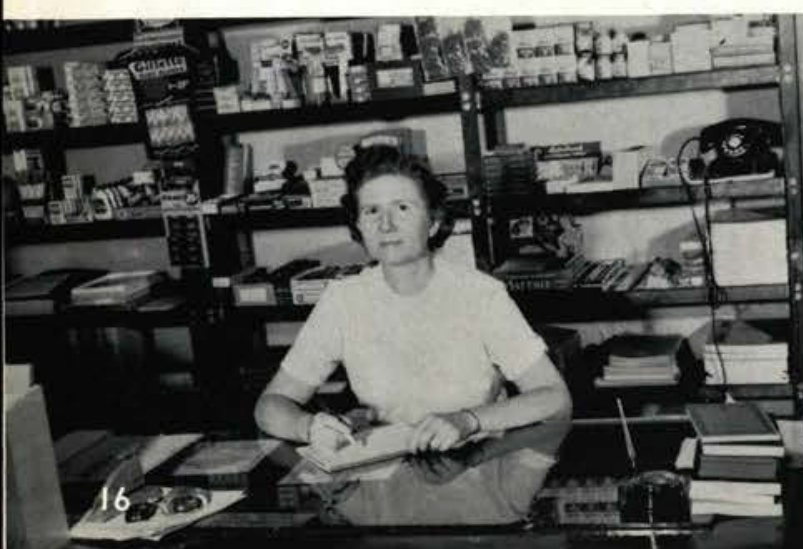

LOIS JEREMIAH, FRAN COOPER, Assistants

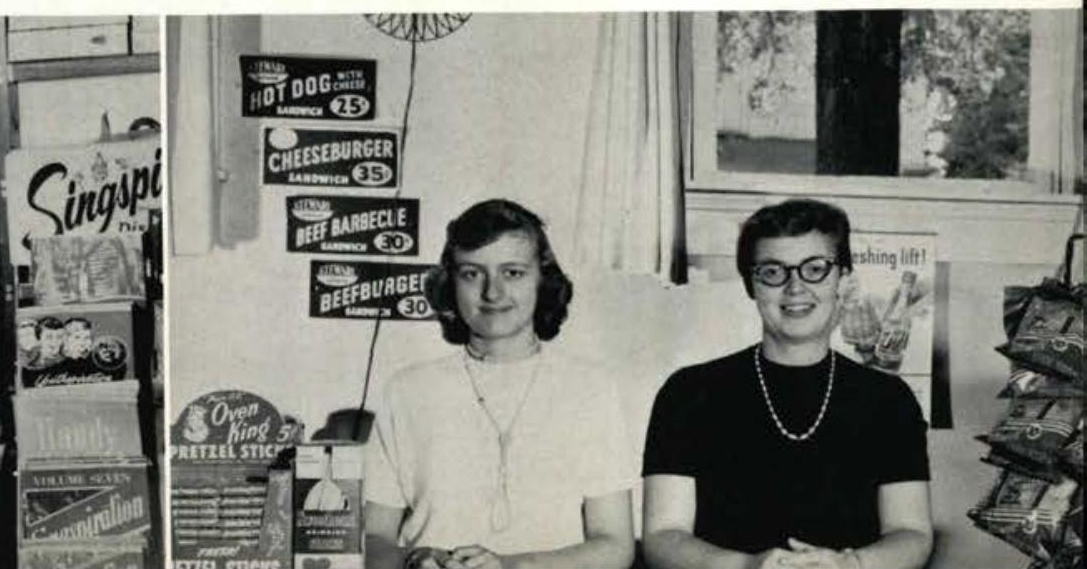




\section{Kitchen Staff}

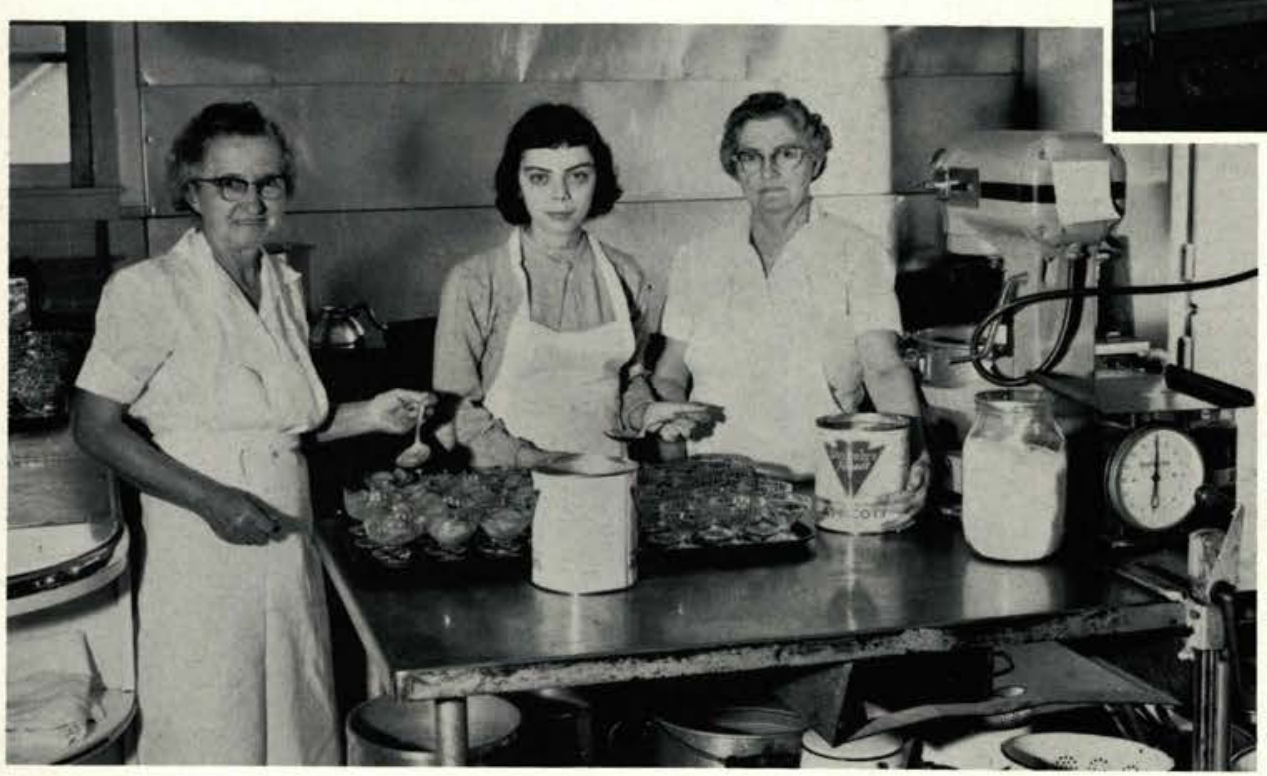

MRS. SHIRLEY

Cafeteria Manager

MRS. UNDERWOOD, LINDA JOHNSON. MRS. FRIBERG

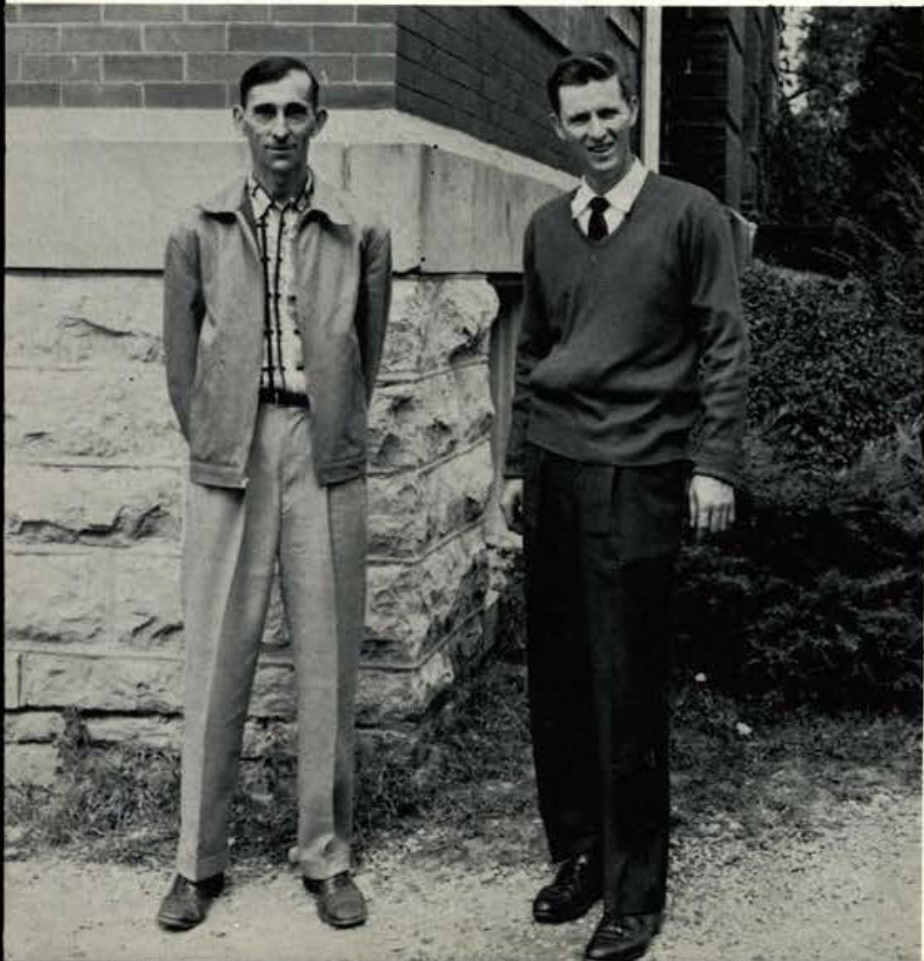

Maintenance

MR. MARSHALL, CLIFF BOESSEL 
Dor - 350 $+\infty$ Dot 2 ? 4212

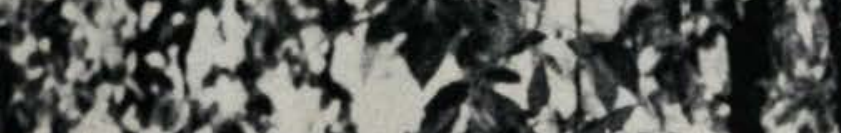
10201 8

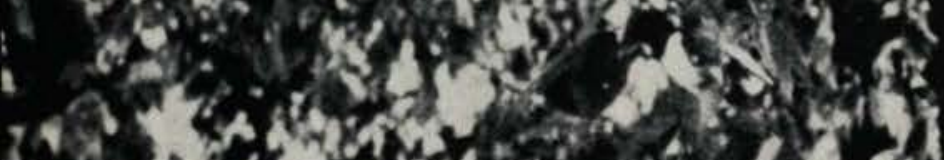

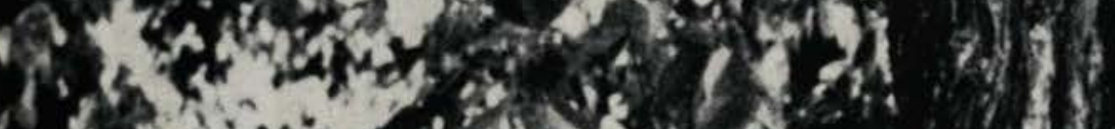

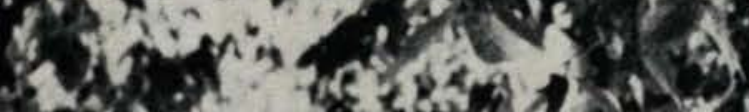

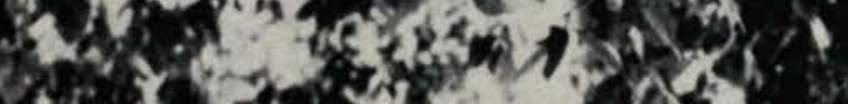

and $x=0,3 x$, nes

7 tith on

4. $3 x^{3} \times 5 x^{2}$

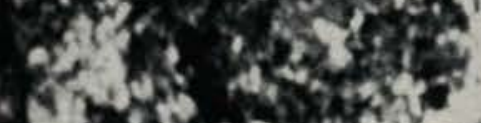

etgras?

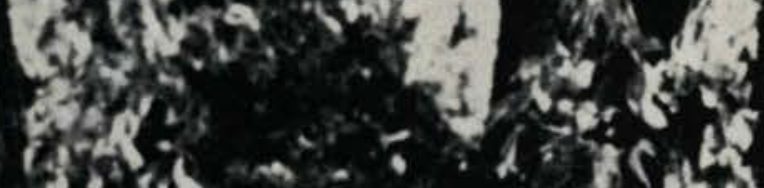

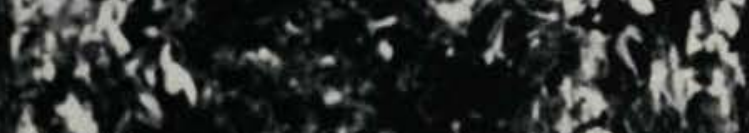

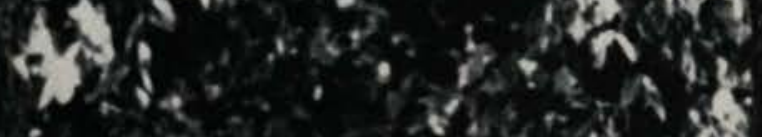

?

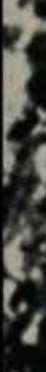

$\therefore$

4

?

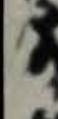
4

$+2$

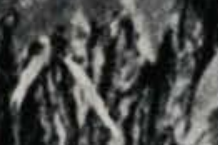

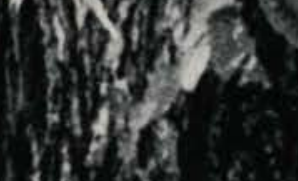

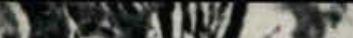

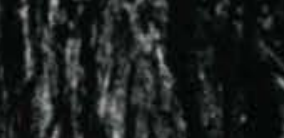

211

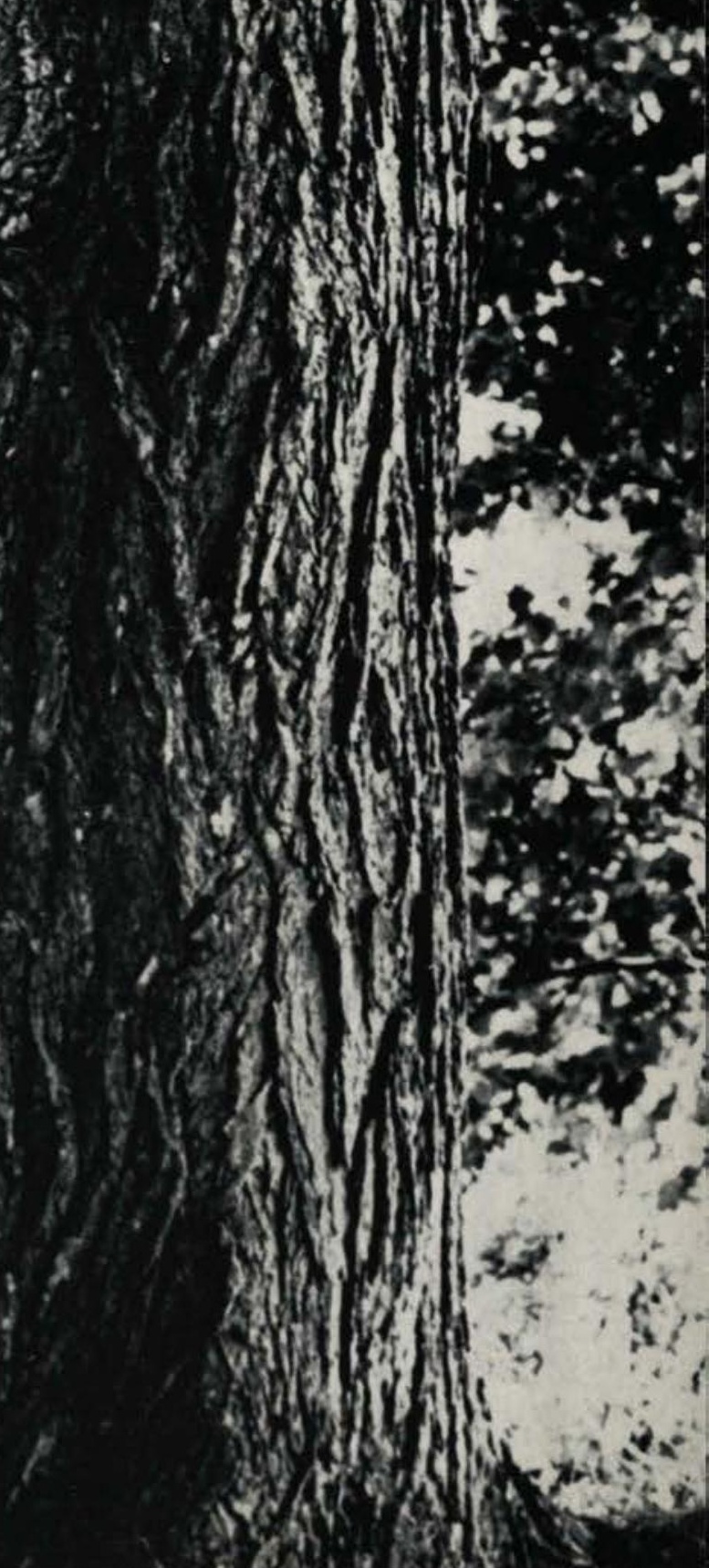




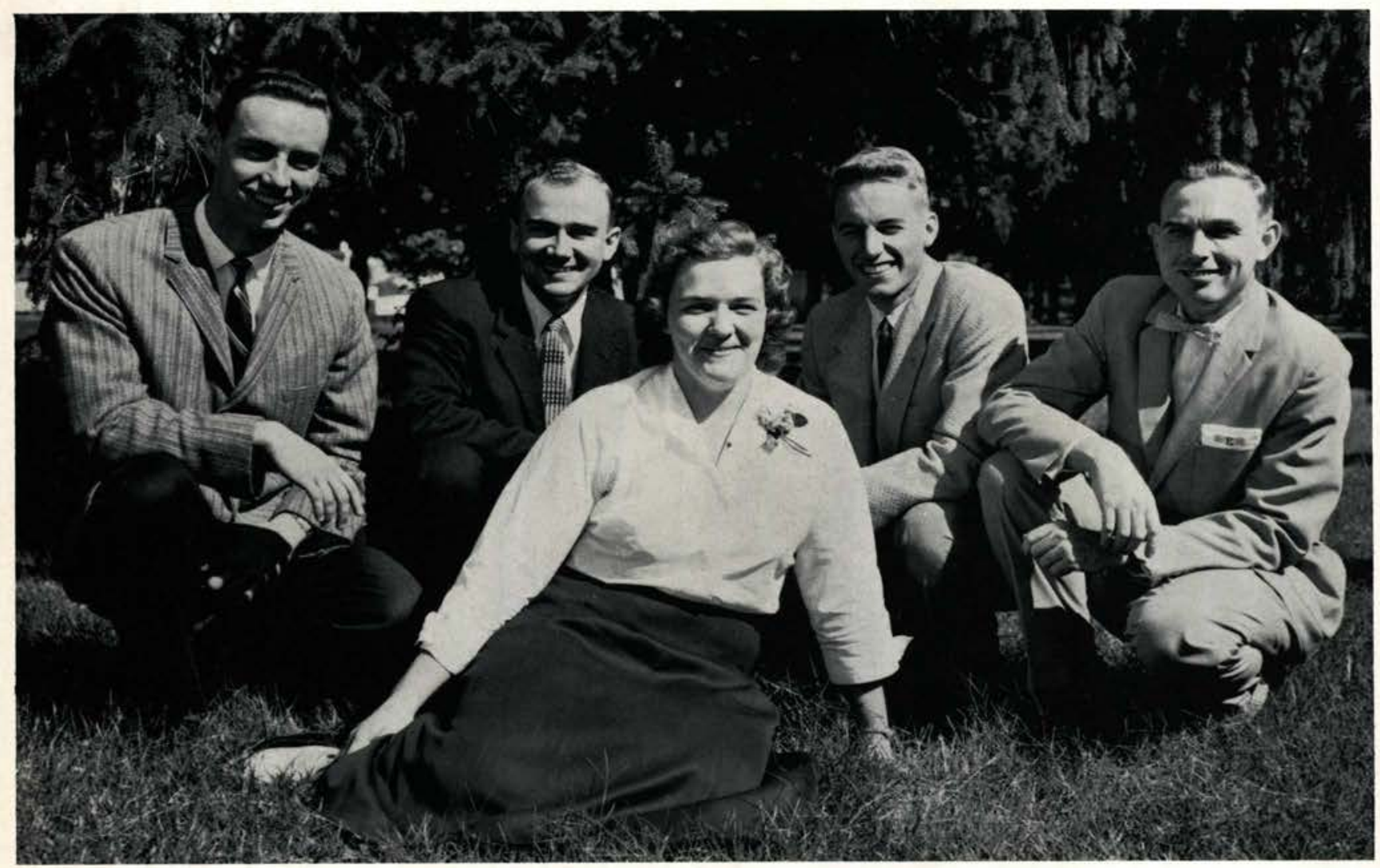

JERRY THORNTON President MAURICE STONE Vice President ESTHER CHESEBRO Secretary ELMER EVANS Treasurer EDWARD MAWHORTER Chaplain

"That in all things He might have the pre-eminence." 


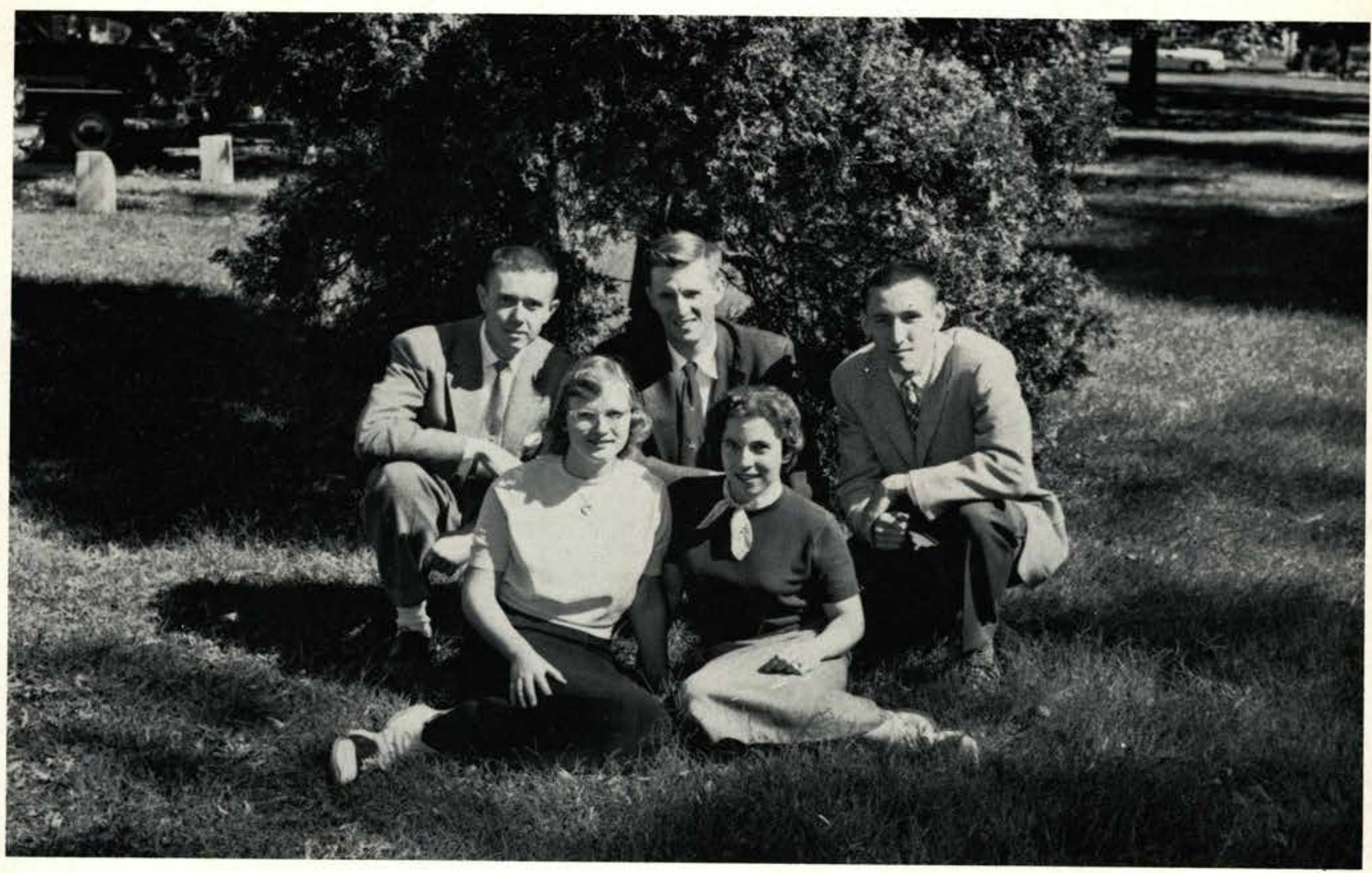

CLIFF BOESSEL

President

BOB HOWDER

Vice President

SANDRA MILLIKIN

Secretary

CAROL JOHNSON

Treasurer

LESTER WEBSTER

Chaplain

"The life that goes forward triumphs."

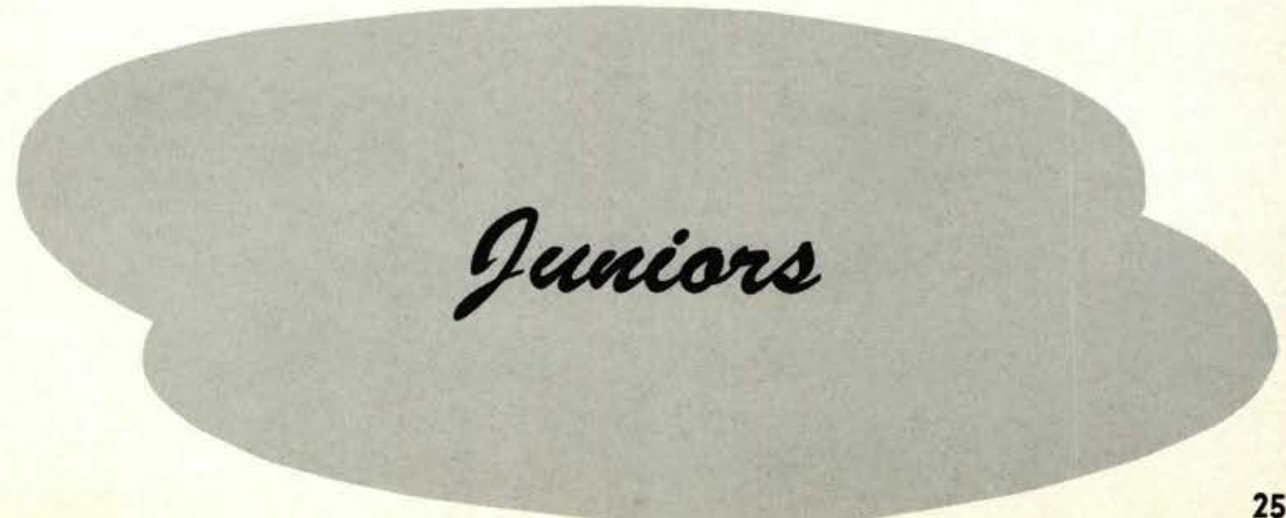




\section{JOHN ABUHL \\ Ankeny, Iowa \\ CLIFFORD BOESSEL \\ Hobart, Indiana}

\section{ALBERTA CARR \\ Hayward, California}

EUGENE CHRISTIAN

Cedarville, Ohio

EVERETT FOX

Naples, New York

ROBERT HOWDER

Angola, New York
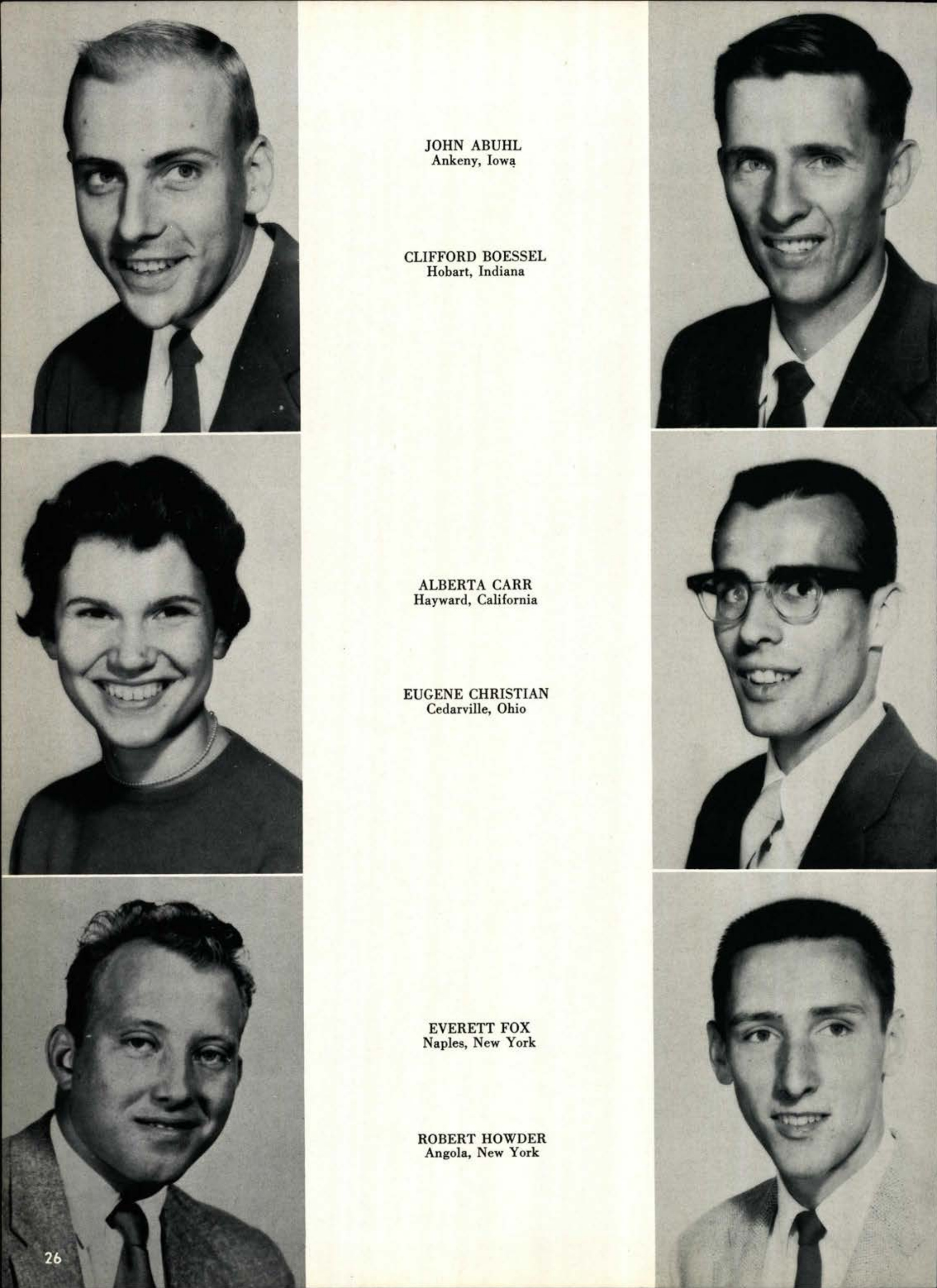


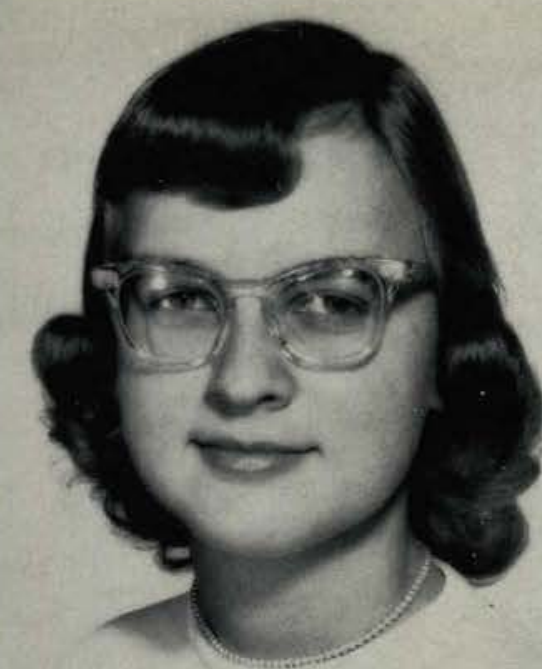

CAROL JOHNSON

West Palm Beach, Florida

SANDRA MILLIKIN

Kalkaska, Michigan

KENNETH PIERPONT

Newark, Ohio

JERROLD ROOT

Beaver Dams, New York

THOMAS SHELOW

Altoona, Pennsylvania

DAVID THOMAS

Dayton, Ohio
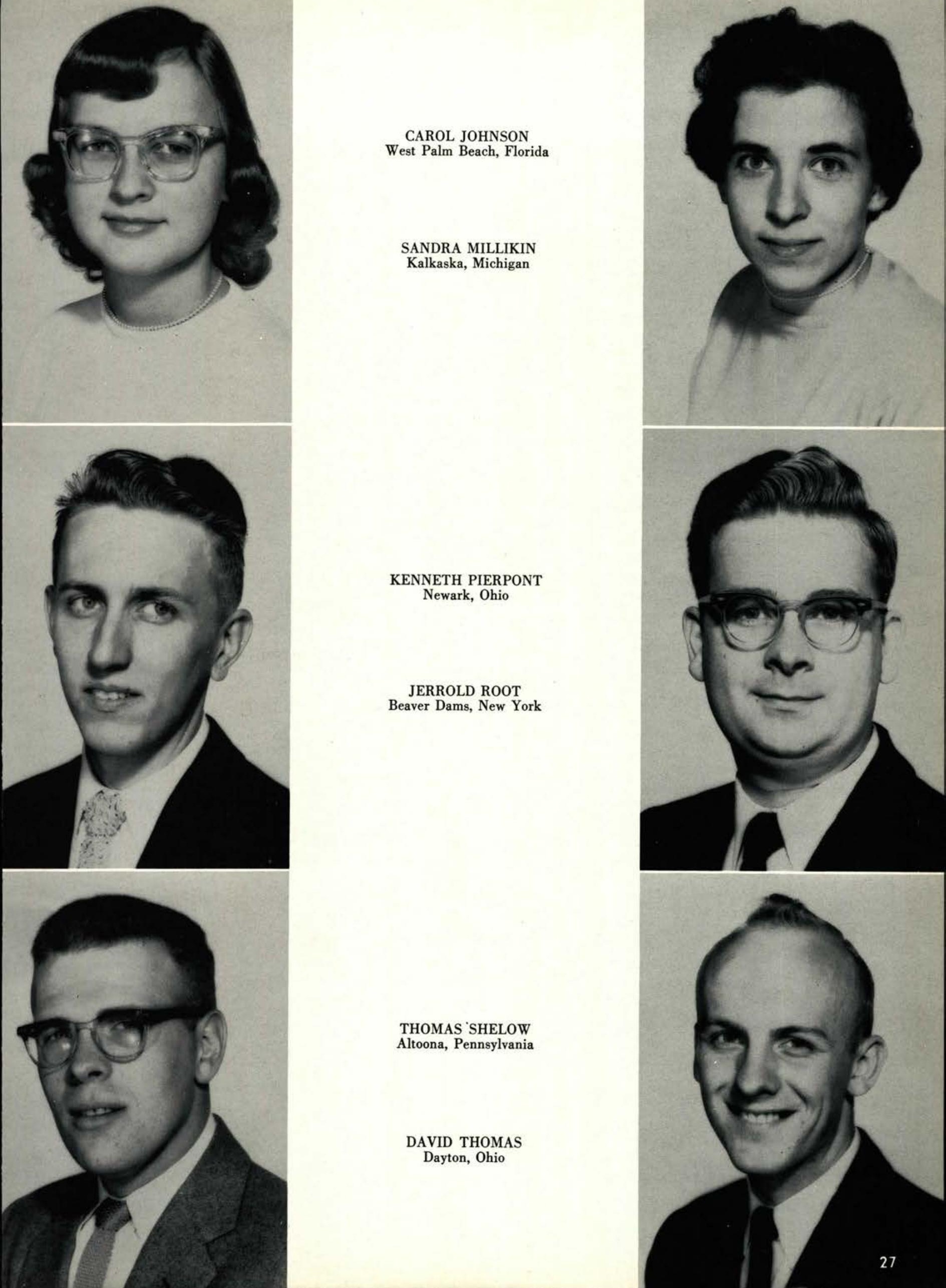
PAUL VAN KLEEK

Lapeer, Michigan

MARSHALL WATSON

Cedarville, Ohio

LESTER WEBSTER

North Boston, New York

GRACE WILLETTS

Berea, Ohio

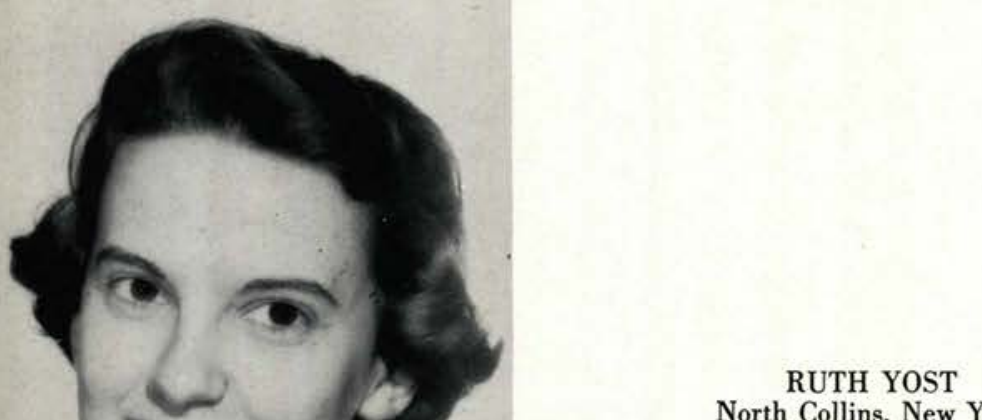

North Collins, New York

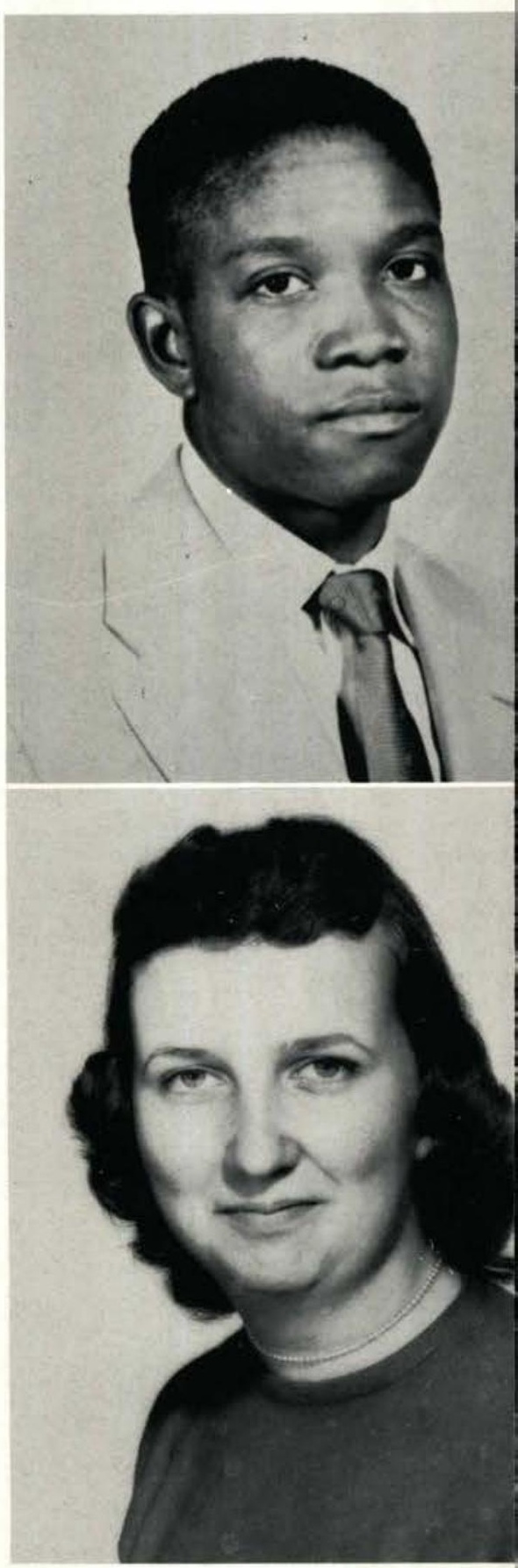




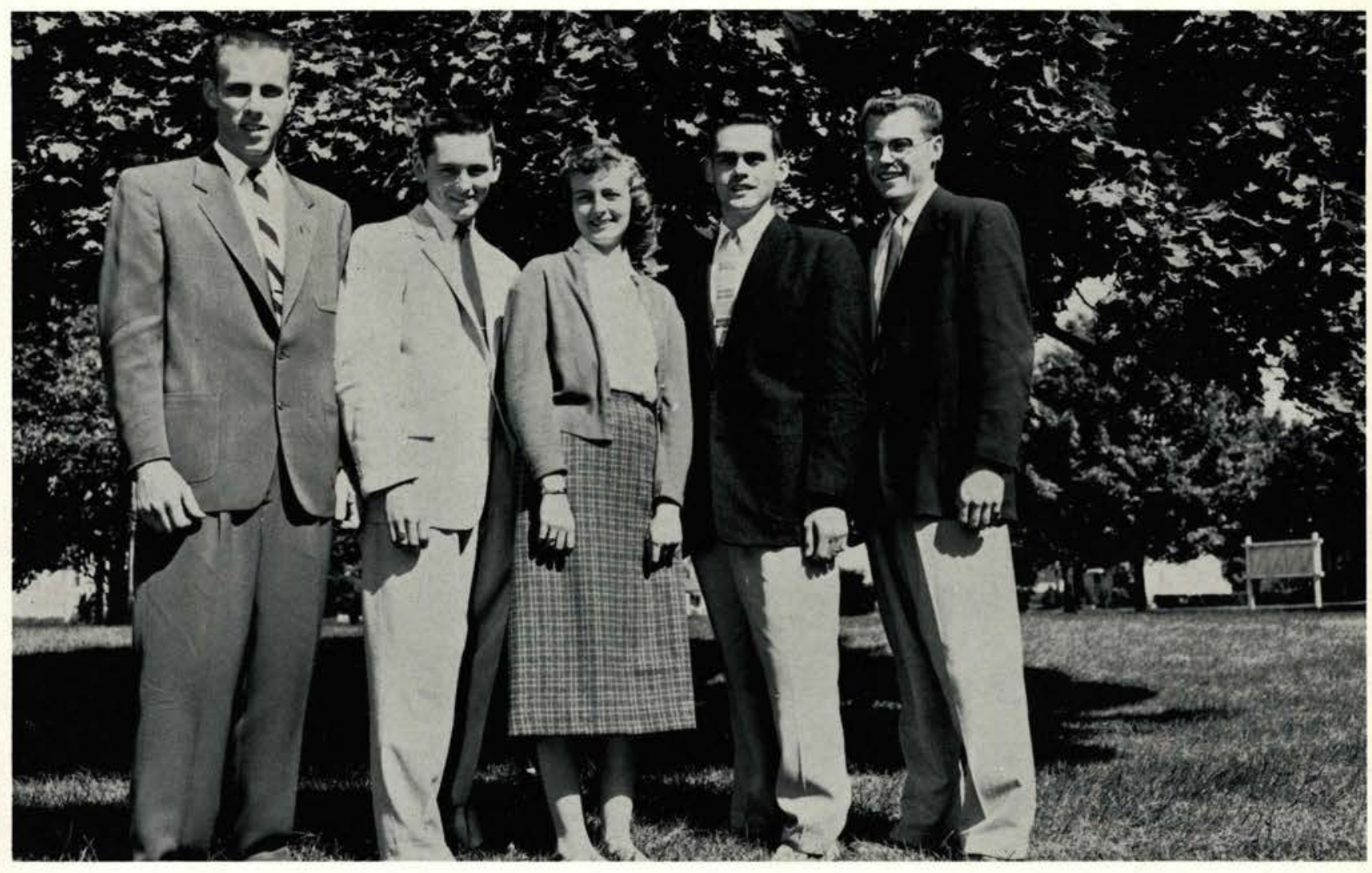

MERLIN AGER President

DWAYNE FRANK Vice President

LOIS JEREMIAH Secretary JOHN ENTNER Treasurer DE ELDA PAYTON Chaplain

"To God the glory, to men the Gospel."

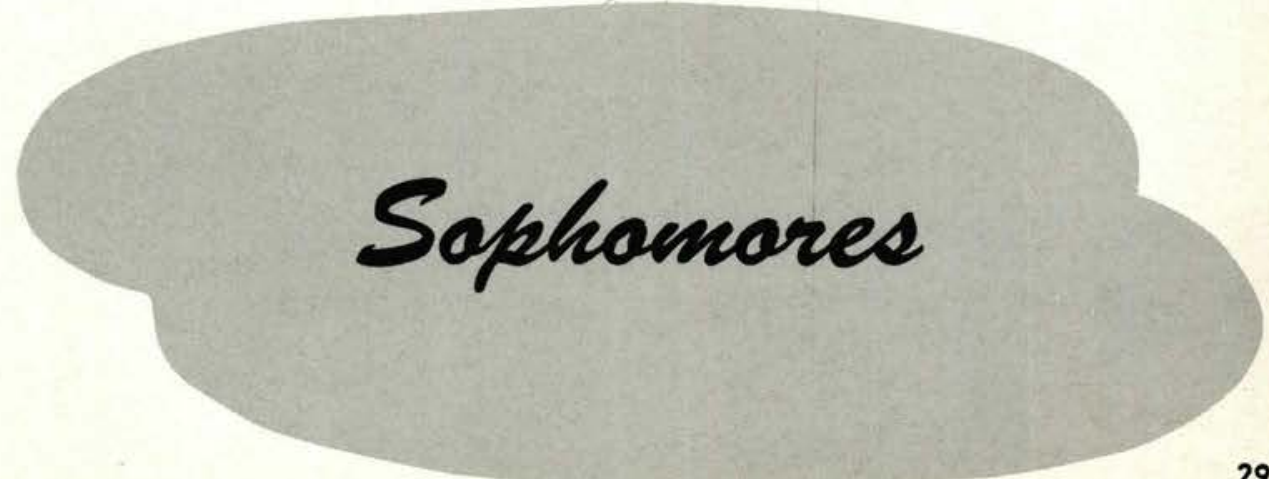


MERLIN AGER

Verona, Wisconsin

PAUL ANDERSON

Gary, Indiana

ROY CARR

Sinclairville, New York

ALBERTA CHAFFE

Thompson, Ohio

STUART CHAFFE

Thompson, Ohio

GORDON CHRISTIAN

Belle Center, Ohio

NEVA CLAYPOOL

Kittanning, Pennsylvania

KEITH COLLETT

Inkster, Michigan

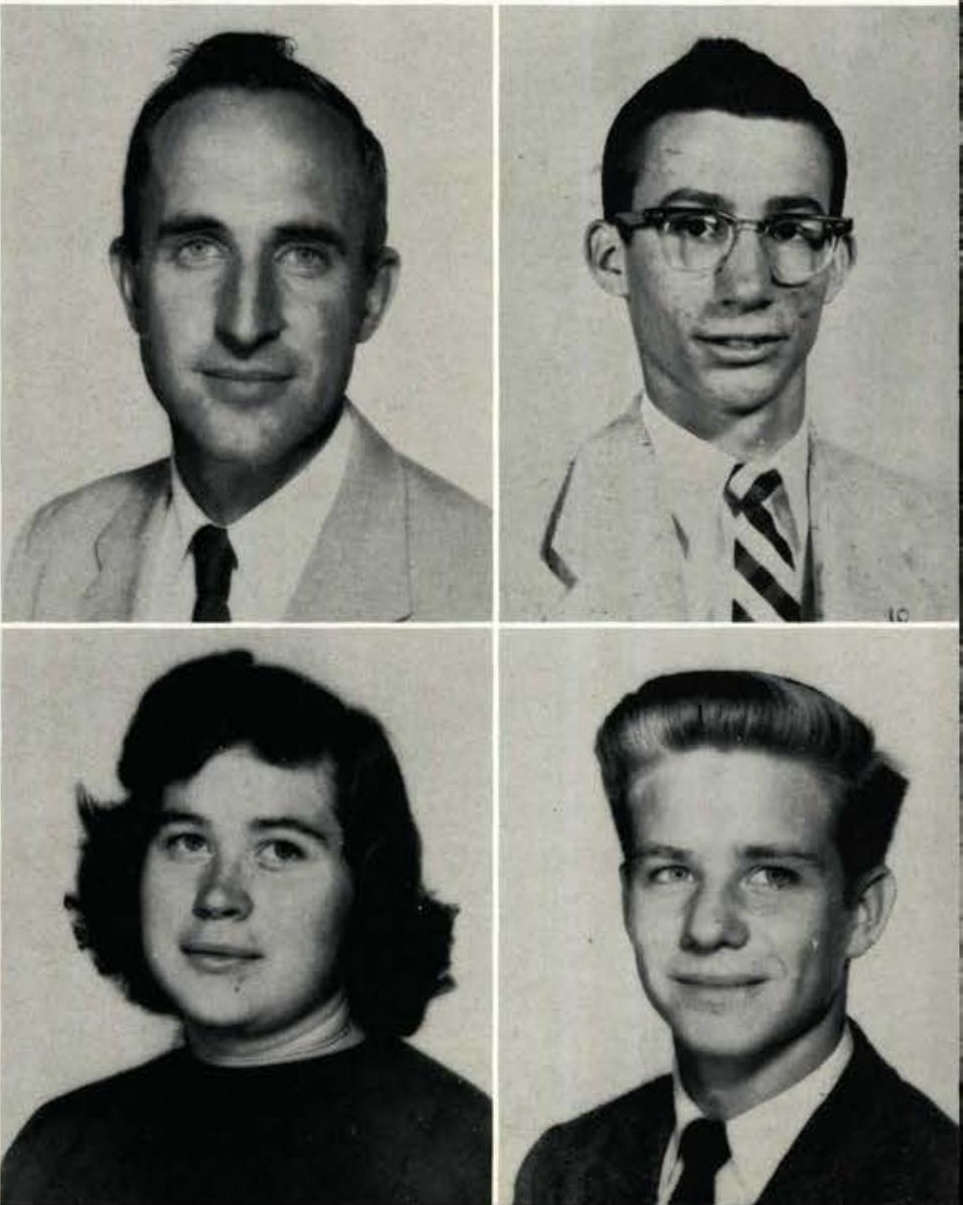


DAVID DAUTEL

Portsmouth, Ohio

KENNETH DOCTOR

Ellsworth, Michigan

JOHN ENTNER

Connersville, Indiana

PHYLLIS ERNST

Fayette, Ohio
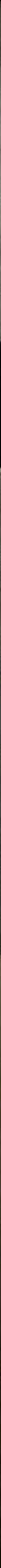

\section{DWAYNE FRANK}

Kuna, Idaho

TIMOTHY GRAFTON

Freeport, Pennsylvania

RICHARD GRAVEN

Vandalia, Illinois

CAROLYN HALE

Toledo, Ohio 


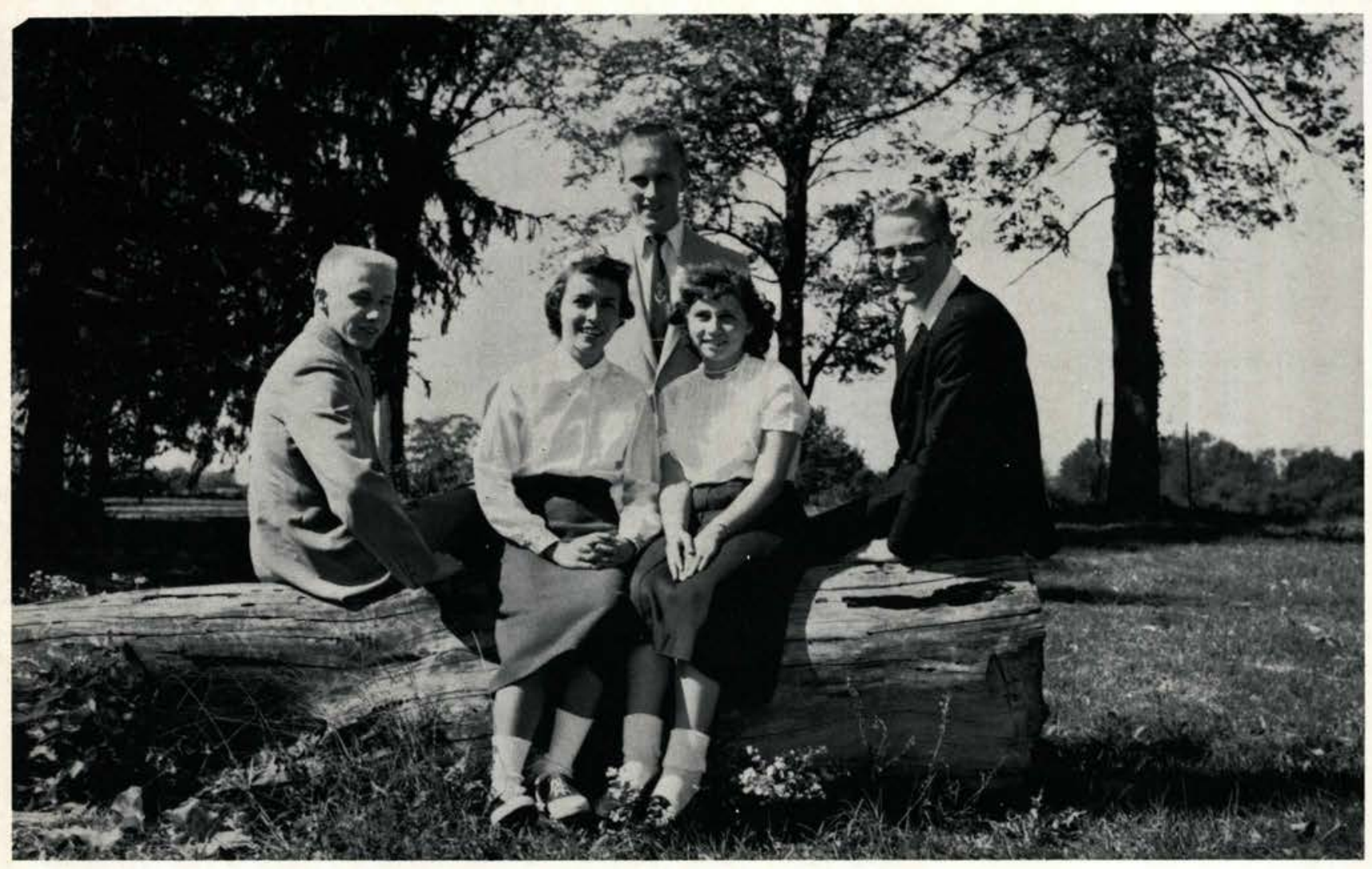

JIM ENTNER

President

DAN PURDY

Vice President

MARLENE DAVIS

Secretary

ESTHER WEISS

Treasurer

DAVE PERRY

Chaplain

"With eternity's values in view"

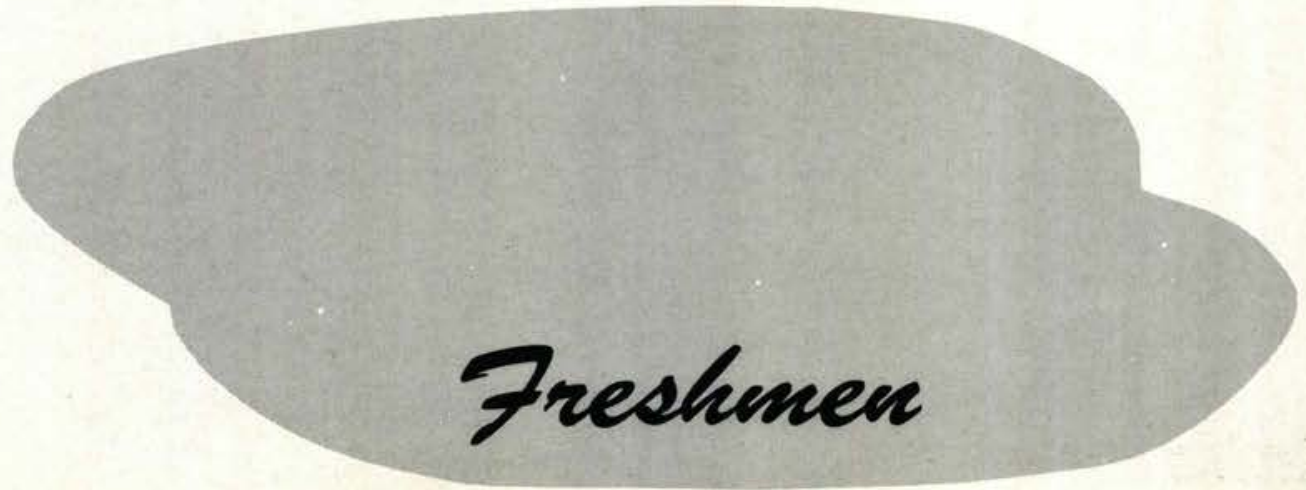




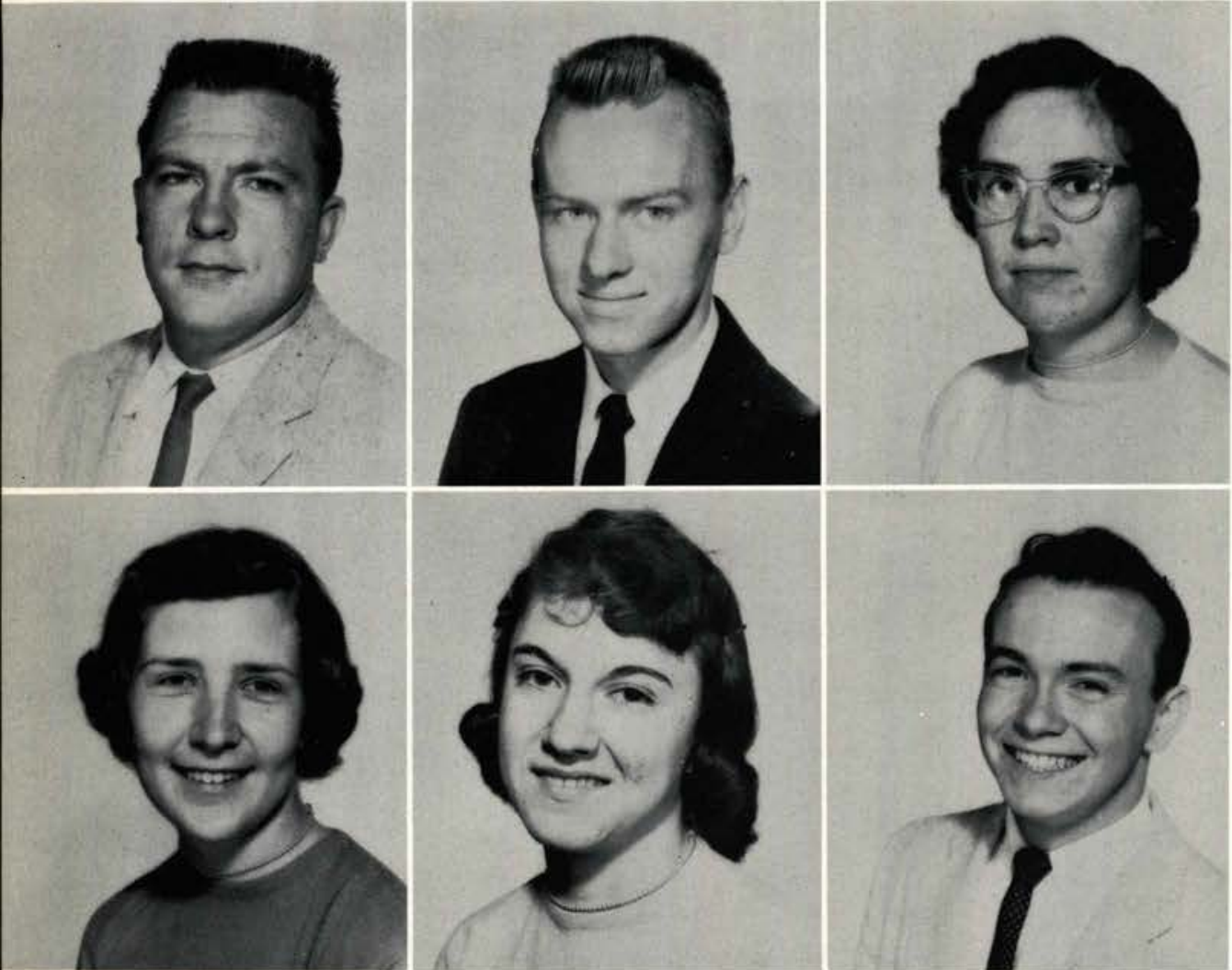

COLBY GOODRICH

Lincoln Center, Maine

TERRY GOODRICH

Boise, Idaho

JOYCE GRANT

East Moline, Illinois

\section{SHIRLEY HARRINGTON}

Watseka, Illinois

RUTH HIMSEL

Verona, Wisconsin

JOHN INGRAM

Columbus, Ohio

RICHARD JACOBS

Flint, Michigan

LINDA JOHNSON

Findlay, Ohio

MAURINE LARSEN

Ames, Iowa

BEVERLY LYON

Crawfordsville, Indiana

DAVID MATSON

Plymouth, Indiana

DEAN MAYO

Cedarville, Ohio
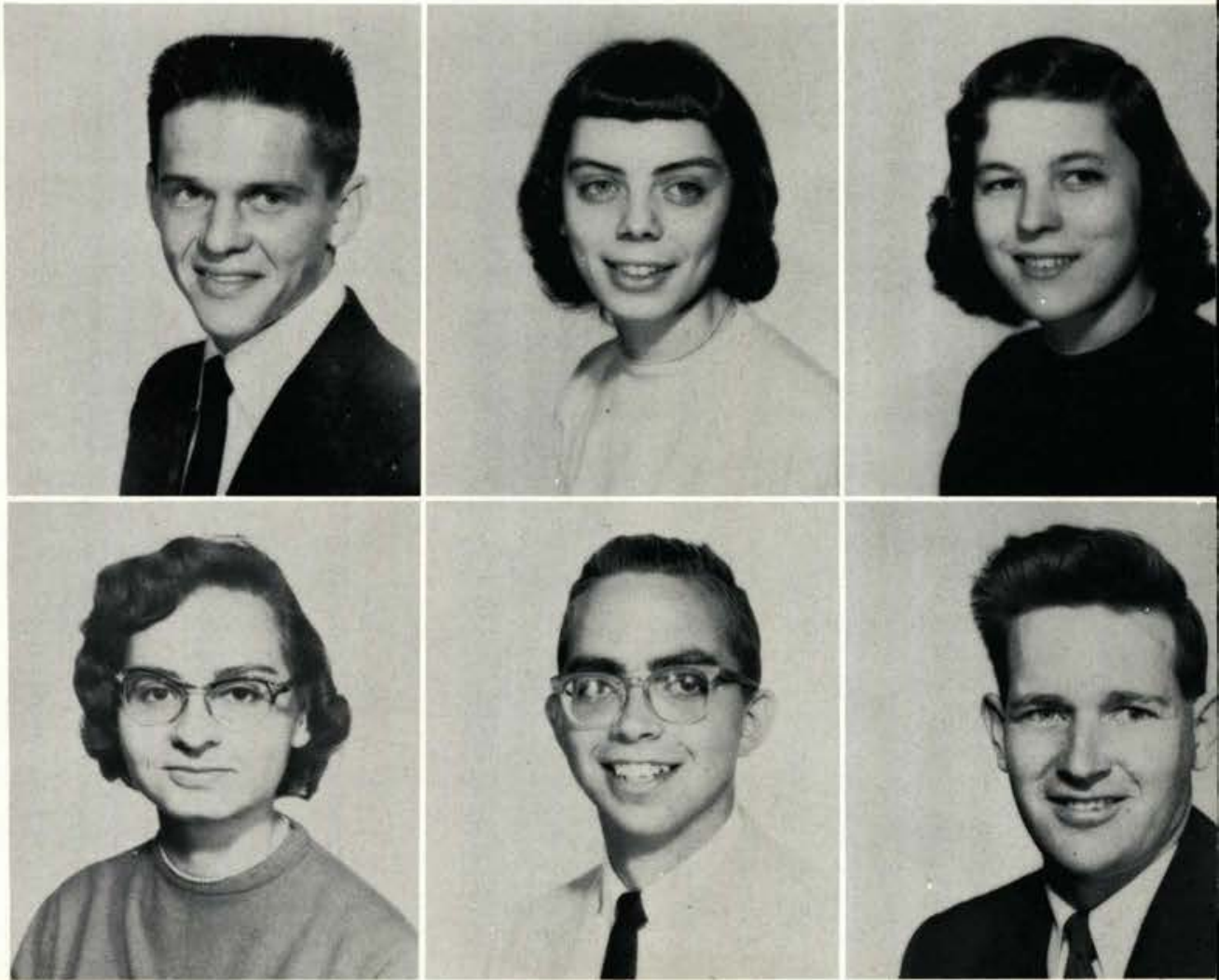
NANCY McDIVITT

Garrettsville, Ohio

NORMAN MOORE

Dayton, Ohio

DELORES OSBORN

Westminster, Colorado

VERN PECK

Cass City, Michigan

DAVID PERRY

Homer, Alaska

PATSY PETRY

New Madison, Ohio

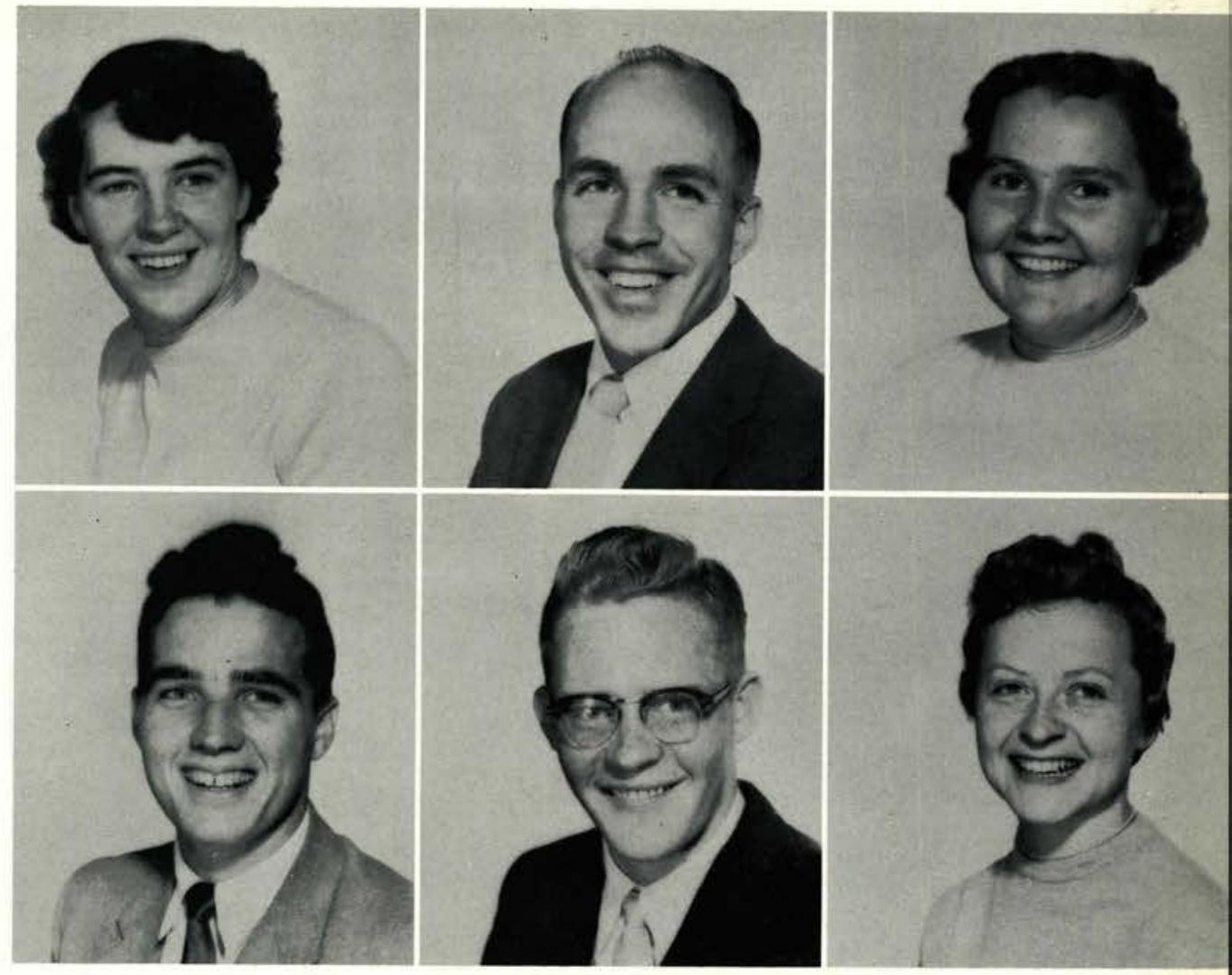

DANIEL PURDY

Columbus Station, Ohio

LON REISING

Cedarville, Ohio

HAROLD RODIN

Burlington, Iowa

NANCY SCHOLTEN

Columbus, Ohio

GLENN SEEVERS

Cleveland, Ohio

BOB SELF

Toledo, Ohio 

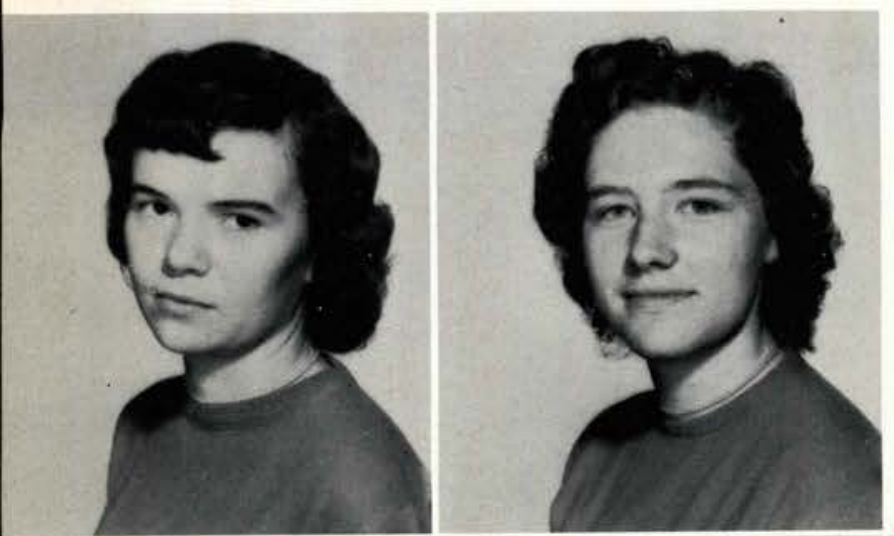

LOREEN SLAUGHENHAUPT

Springville, New York

GLENDA SLAVENS

Crawfordsville, Indiana
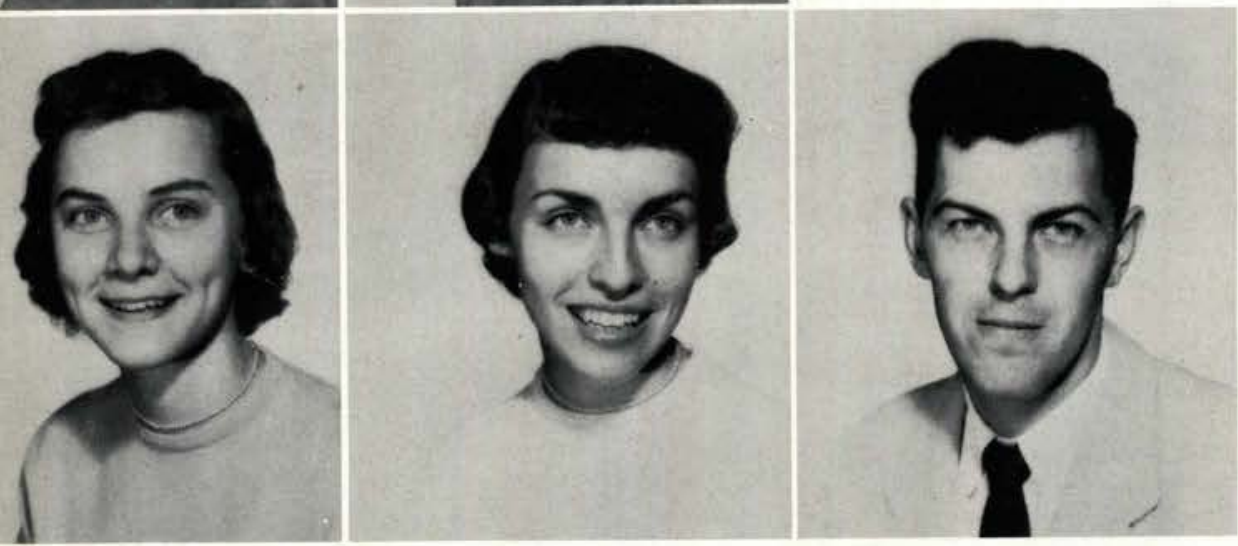

BEVERLY VAN MULLEN

Marne, Michigan

ESTHER WEISS

Fairview Park, Ohio

WILLARD WENTZEL

Cedarville, Ohio

MARVIN WISEMAN Springfield, Ohio

LORNA WOOD

Pasadena, California

DEAN ZERBY

Berrien Springs, Michigan

TERRY ZERBY

Berrien Springs, Michigan

CAROL ZOELLNER

Sandusky, Ohio

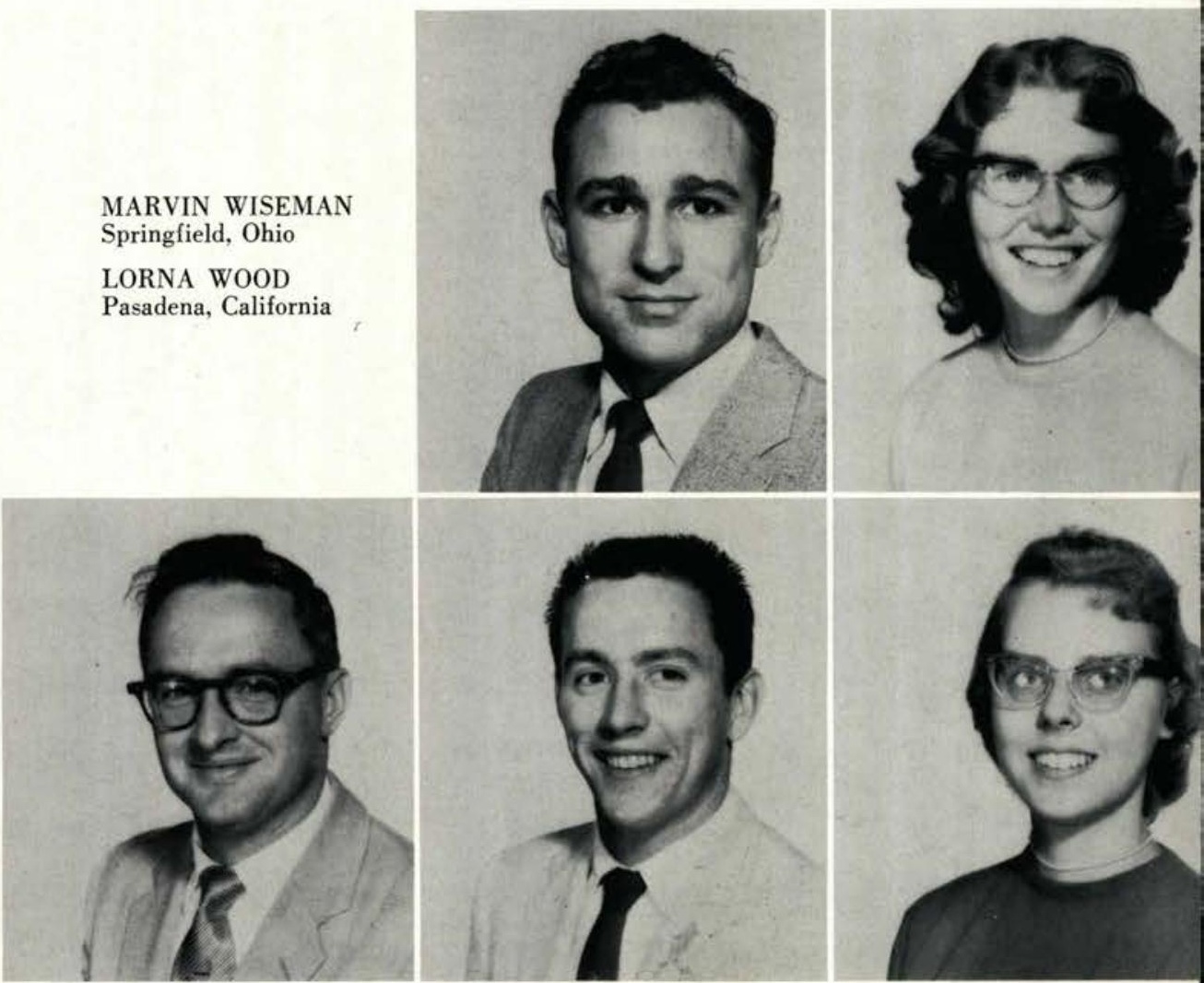




\section{Special Students}

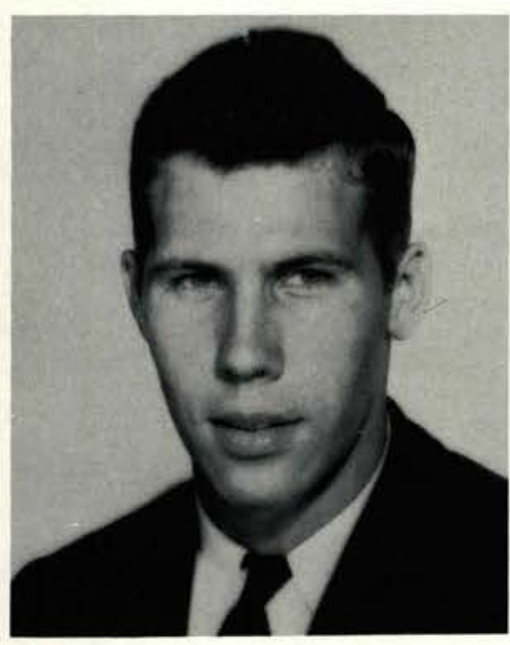

FRED BROOKS

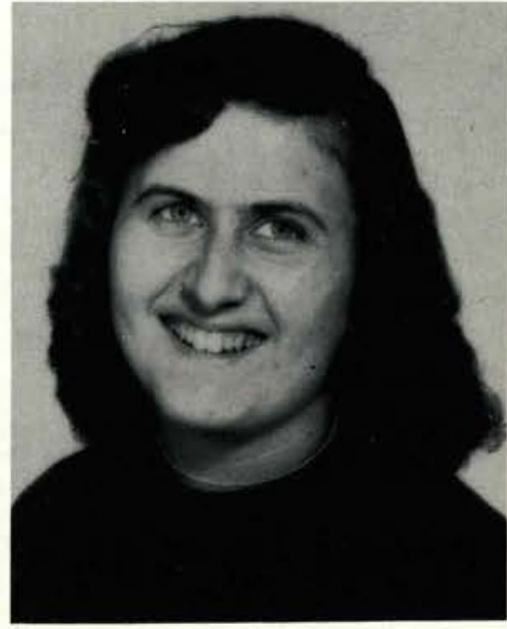

BERNICE COON

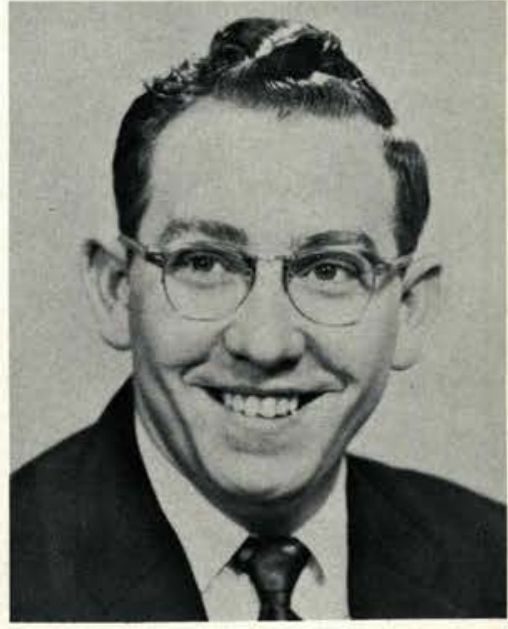

MARTIN EDWARDS

\section{Cedarville College Library and Lannge}

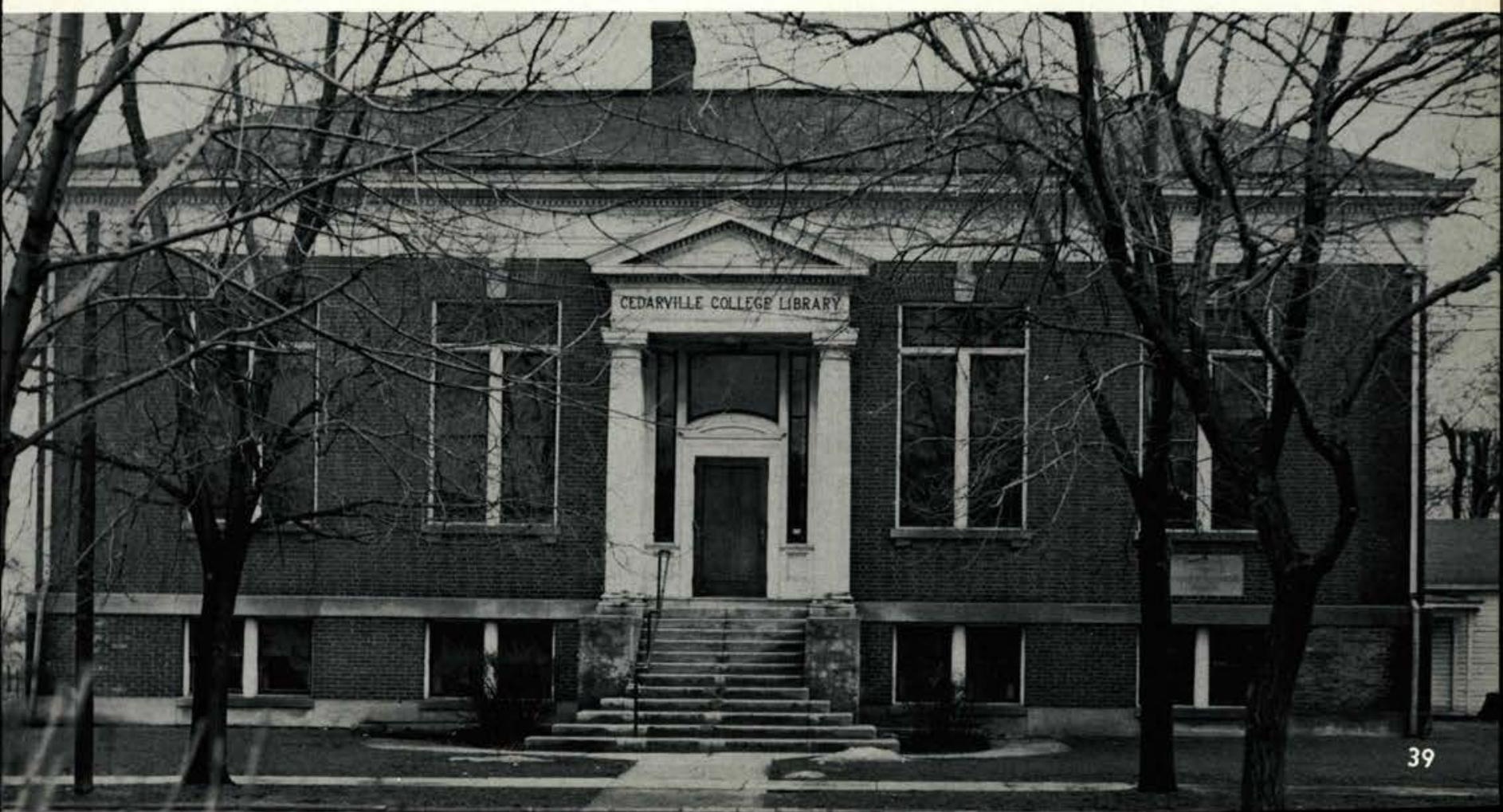




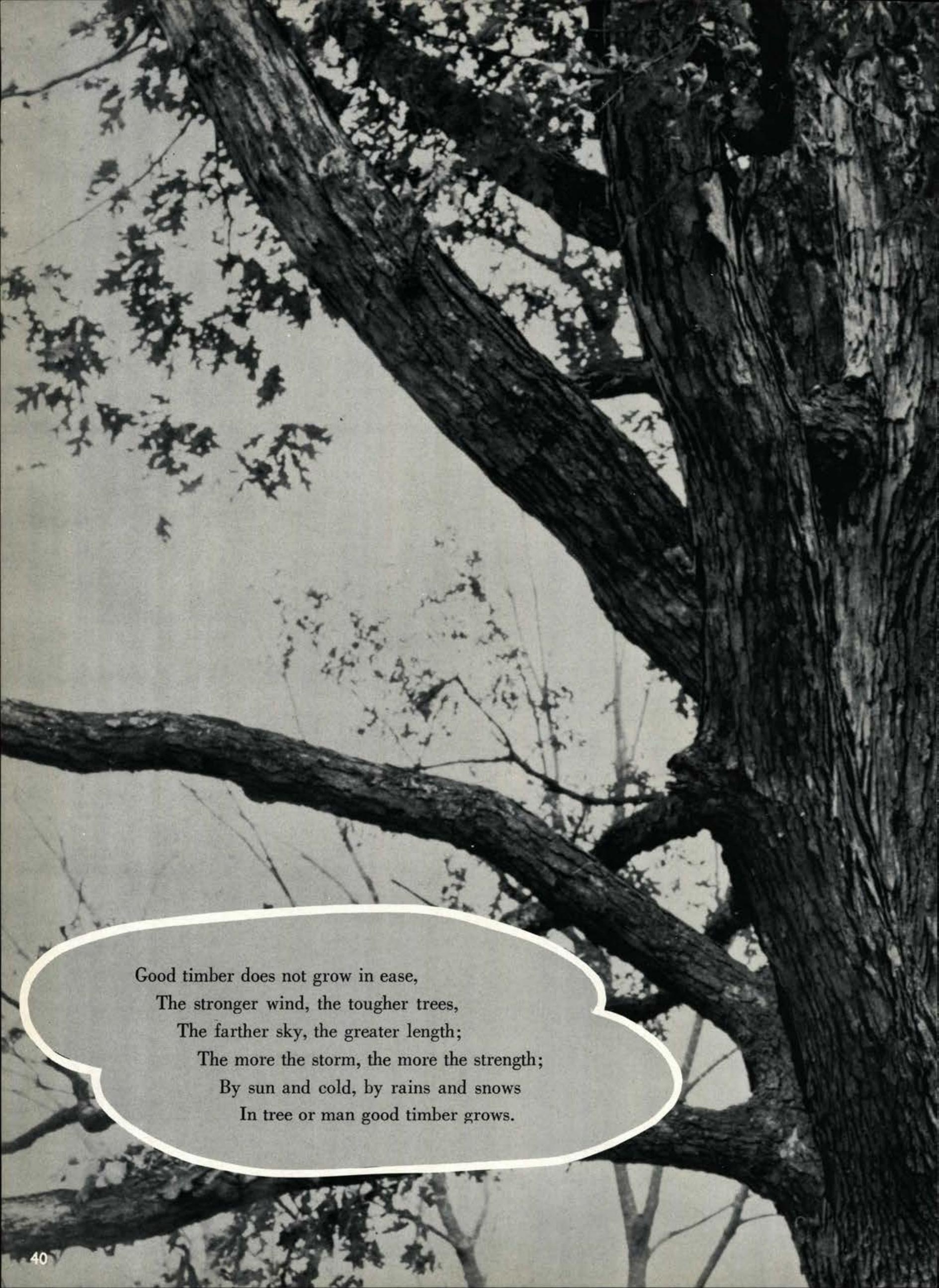





\section{Student Council}

President

Paul VanKleele

Vice President

Gerrey Tharntan

\section{Secretary}

\section{Frances Cooper}

Treasurer

Gohn Entuer

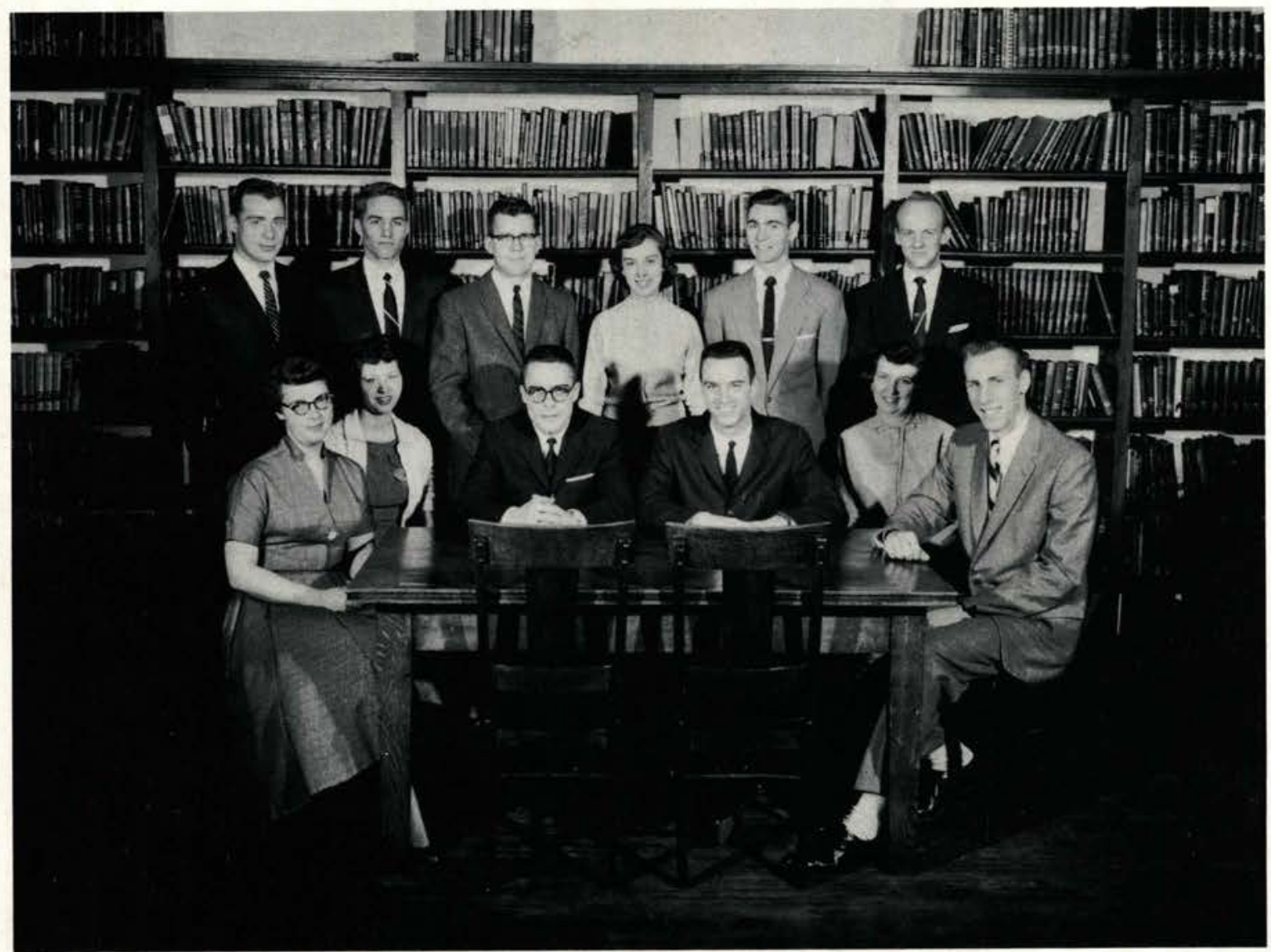

Front row: Frances Cooper, Marcia Crothers, Paul VanKleek, Jerry Thornton, Grace Willetts, John Entner. Back row: Jim Erskin, Maurice Stone, Bob Humphreys, Lucy Lyons, Glenn Seevers, Dave Thomas.

The Student Council is the form of student government composed of members elected from each individual class. Its function is the development of a well-rounded social, spiritual, and academic life for the entire student body. 
"The purpose of the Varsity "C"

Club shall be to promote and encourage intramural and intercollegiate athletics on the Cedarville Campus ...."

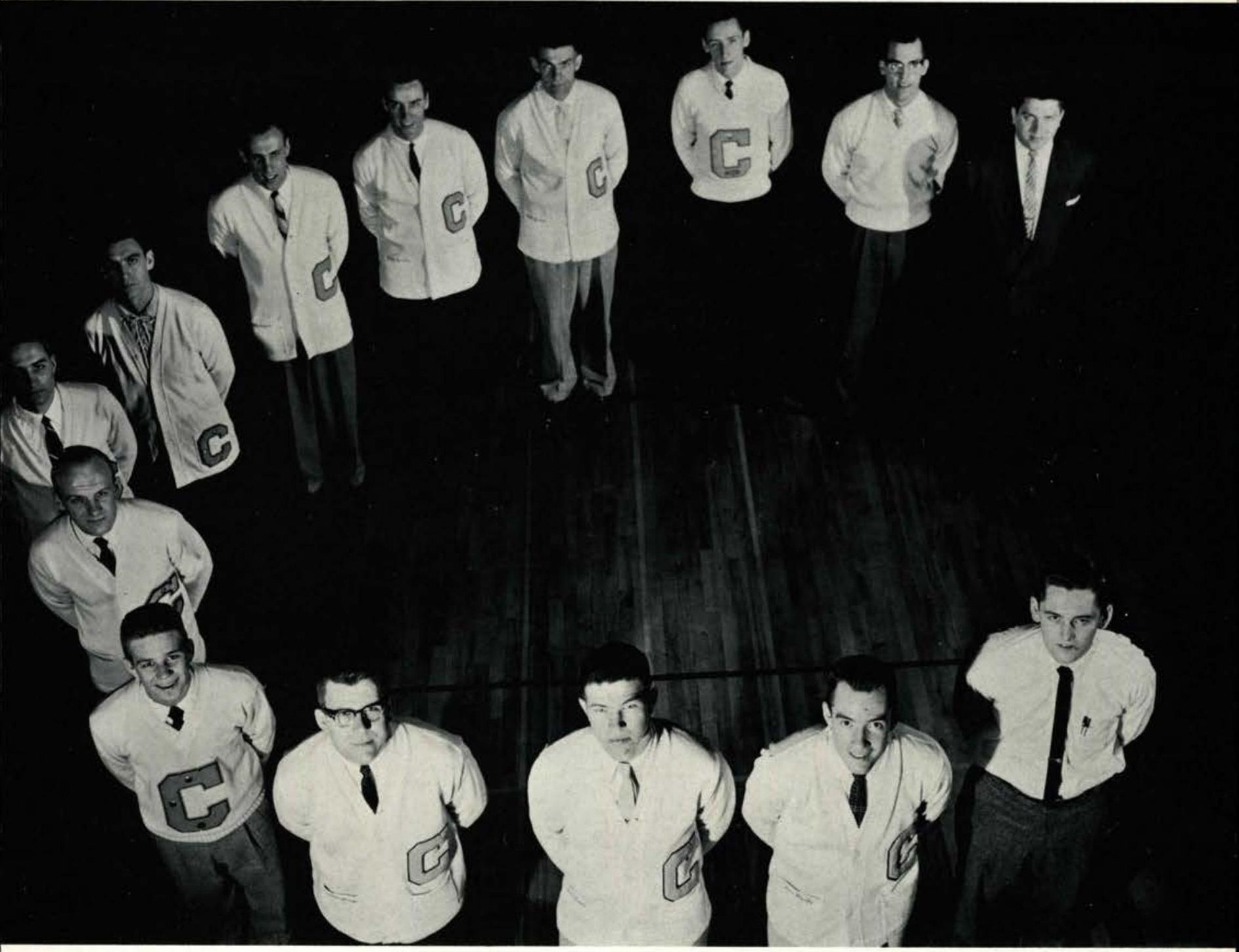

Coach Bowser, Roy Carr, Bob Howder, Dick Wentzel, Jerry Thornton, John Entner, Bob Marcellino, Maurice Stone, Dave Thomas, Keith Collett, Bob Humphreys, Pete Reese, Lane Moody, Merlin Ager.

President DICK WENTZEL

Vice President LANE MOODY
Secretary DAVE THOMAS

Treasurer JERRY THORNTON 


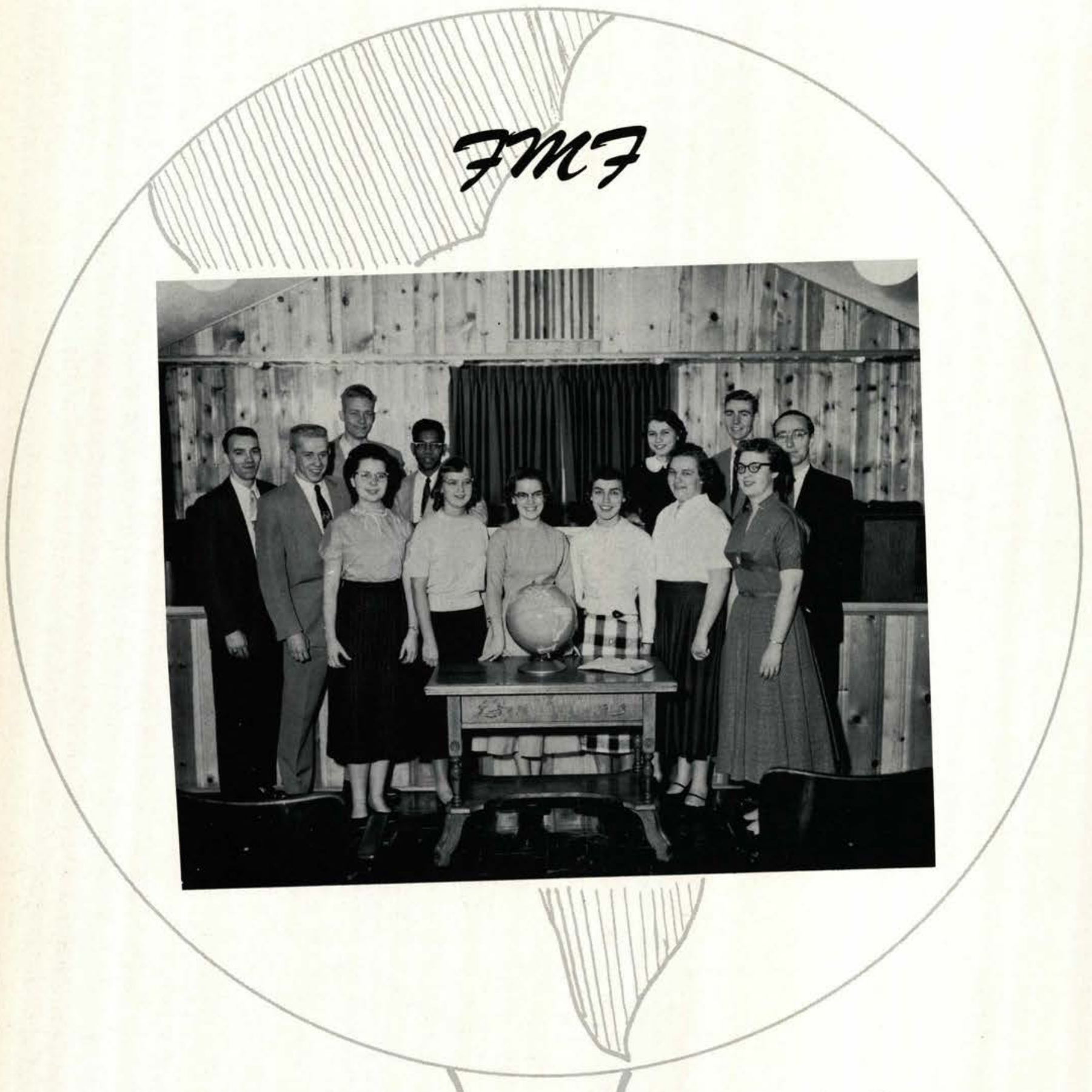

Front row: Allen Foltz, Dan Purdy, President; Helen Stevens, Secretary-Treasurer; Carol Johnson, Ruth Yost, Esther Weiss, Esther Chesebro, Frances Cooper. Back row: Paul Anderson, Hublall Sookram, Marlene Davis, Glenn Seevers, Mr. Bruce Turnbull, Advisor.

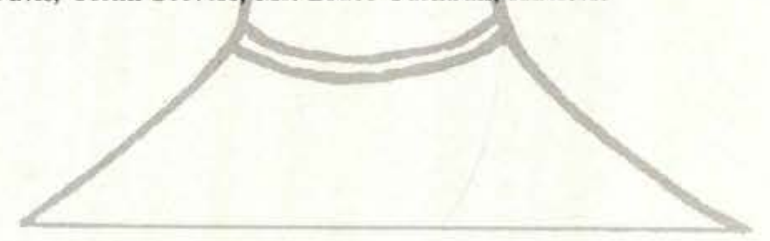

The purpose of the Foreign Missions Fellowship is to stimulate interest in missionary activity. Although the primary function is prayer, a special project this year has been distributing medicine for use on the Mission field. 


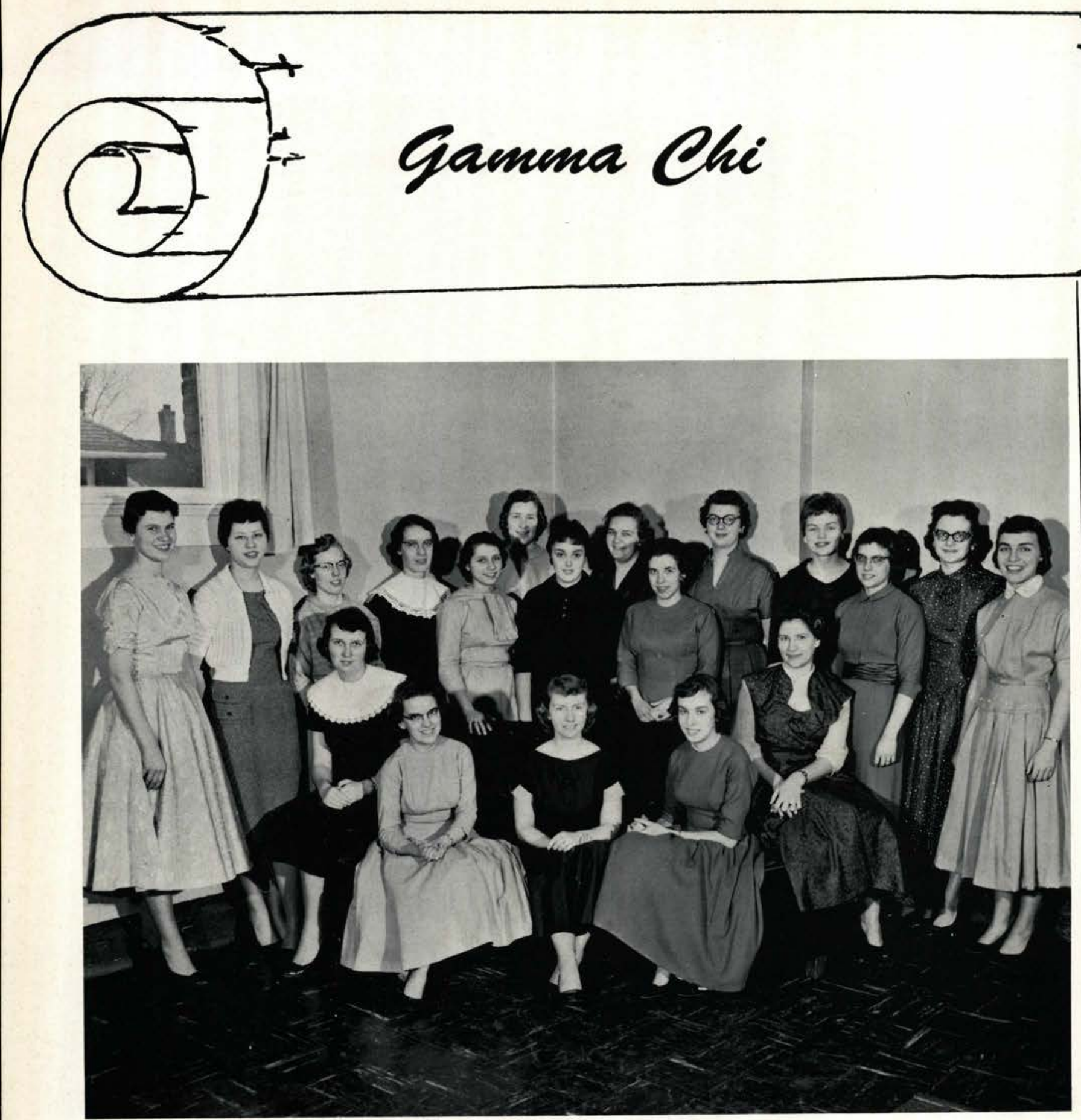

Officers: President, Wanda Horn; Vice President, Ruth Yost; Secretary-Treasurer, Lucy Lyons;

Parliamentarian, Alberta Chafee; Advisor, Mrs. William Ambrose.

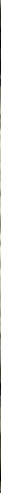




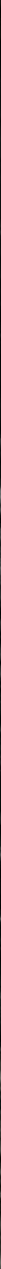




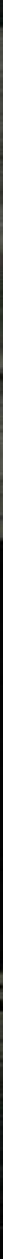

Chair 


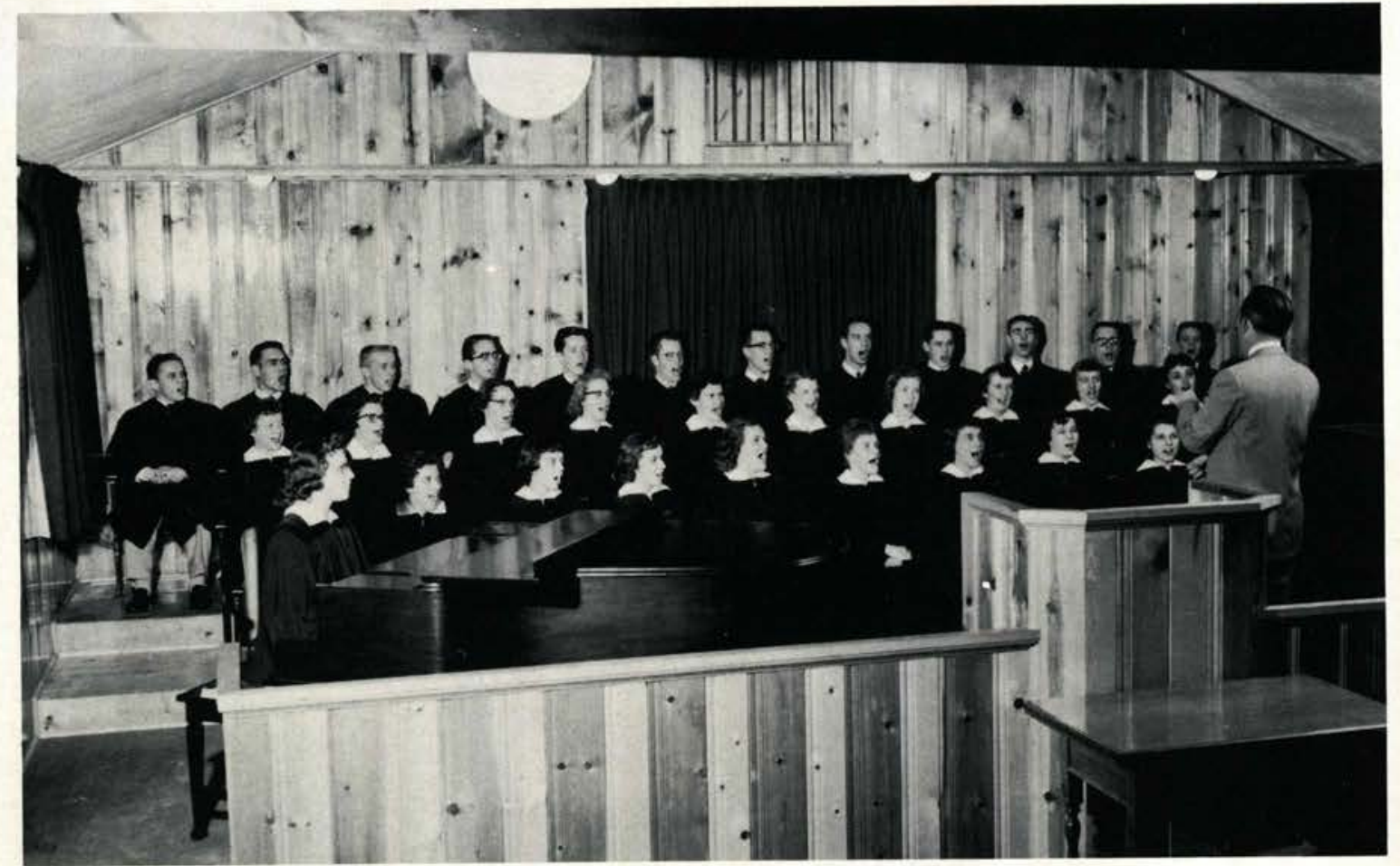

First row: Sandra Millikin, Linda Johnson, Lucy Lyons, Esther Chesebro, Ruth Smelser, Esther Weiss, Maurine Larsen, Marlene Davis. Second row: Patsy Petry, Lois Dodson, Rosemary Smith, Carol Zoellner, Assistant Pianist; Alberta Carr, Barbara Sherry, Shirley Harrington, Grace Willetts, Secretary; Lois Jeremiah, Karen Fay. Third row: Dick Cook, DeElda Payton, President; Dan Purdy, David Matson, Warren Woodard, Dwayne Frank, Roy Carr, Jerry Thornton, Student Director; Merlin Ager, Manager; Glenn Seevers, Paul VanKleek, Keith Collett. Carolyn Hale, Pianist; Mr. William Ambrose, Director.
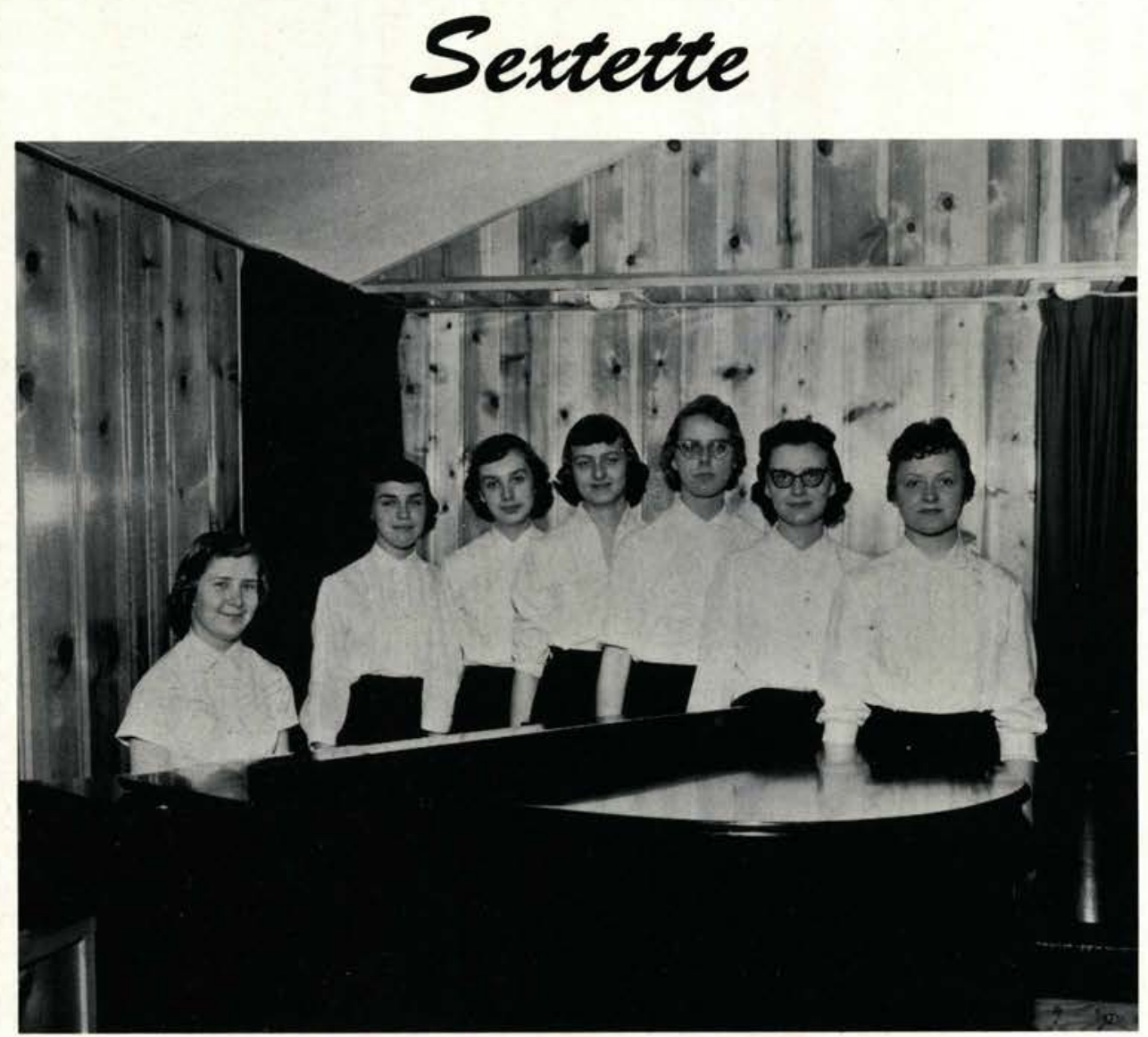

Shirley Harrington, Pianist; Esther Weiss, Lucy Lyons, Lois Jeremiah, Carol Zoellner, Lois Dodson, Patsy Petry. 

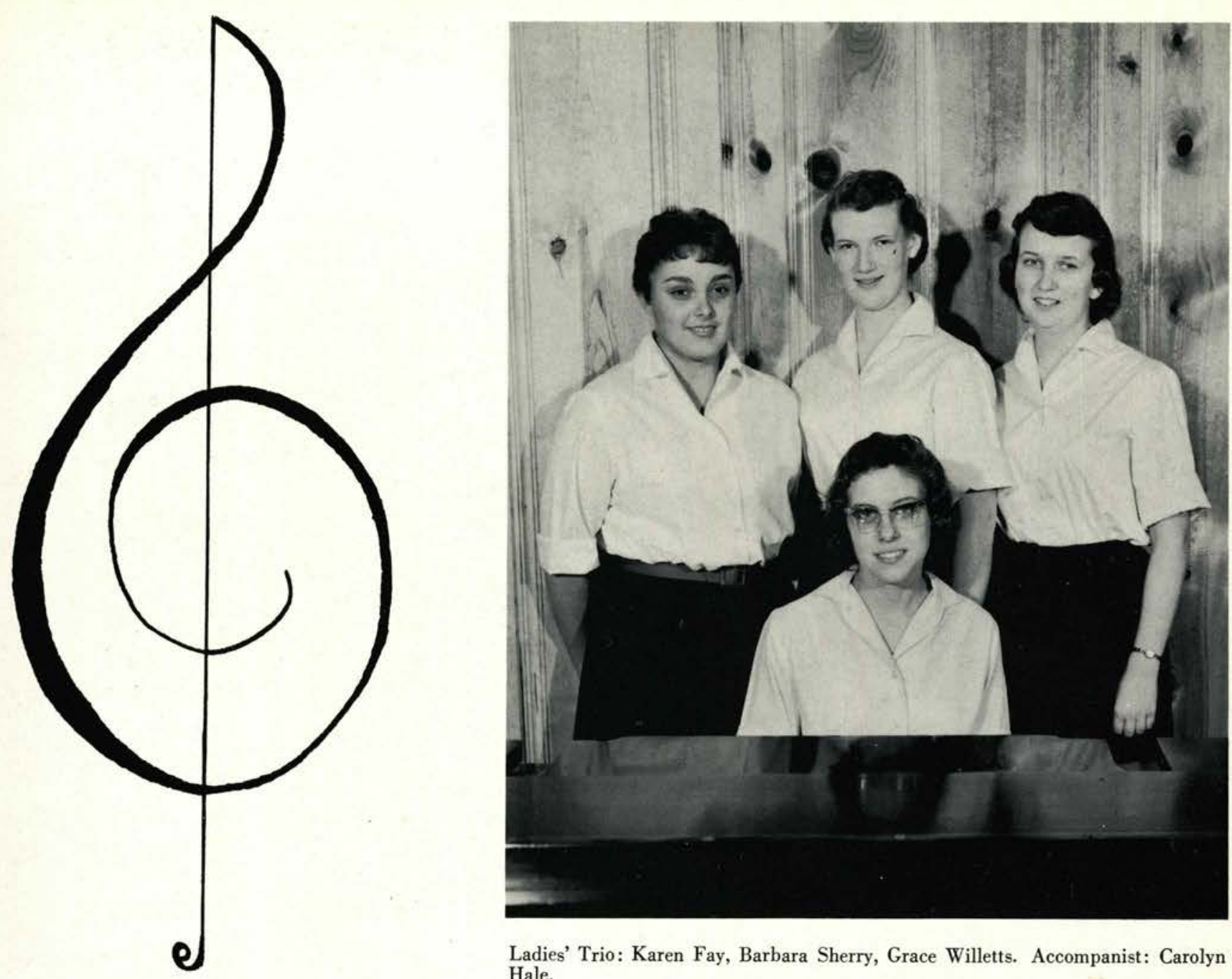

Ladies' Trio: Karen Fay, Barbara Sherry, Grace Willetts. Accompanist: Carolyn Hale.
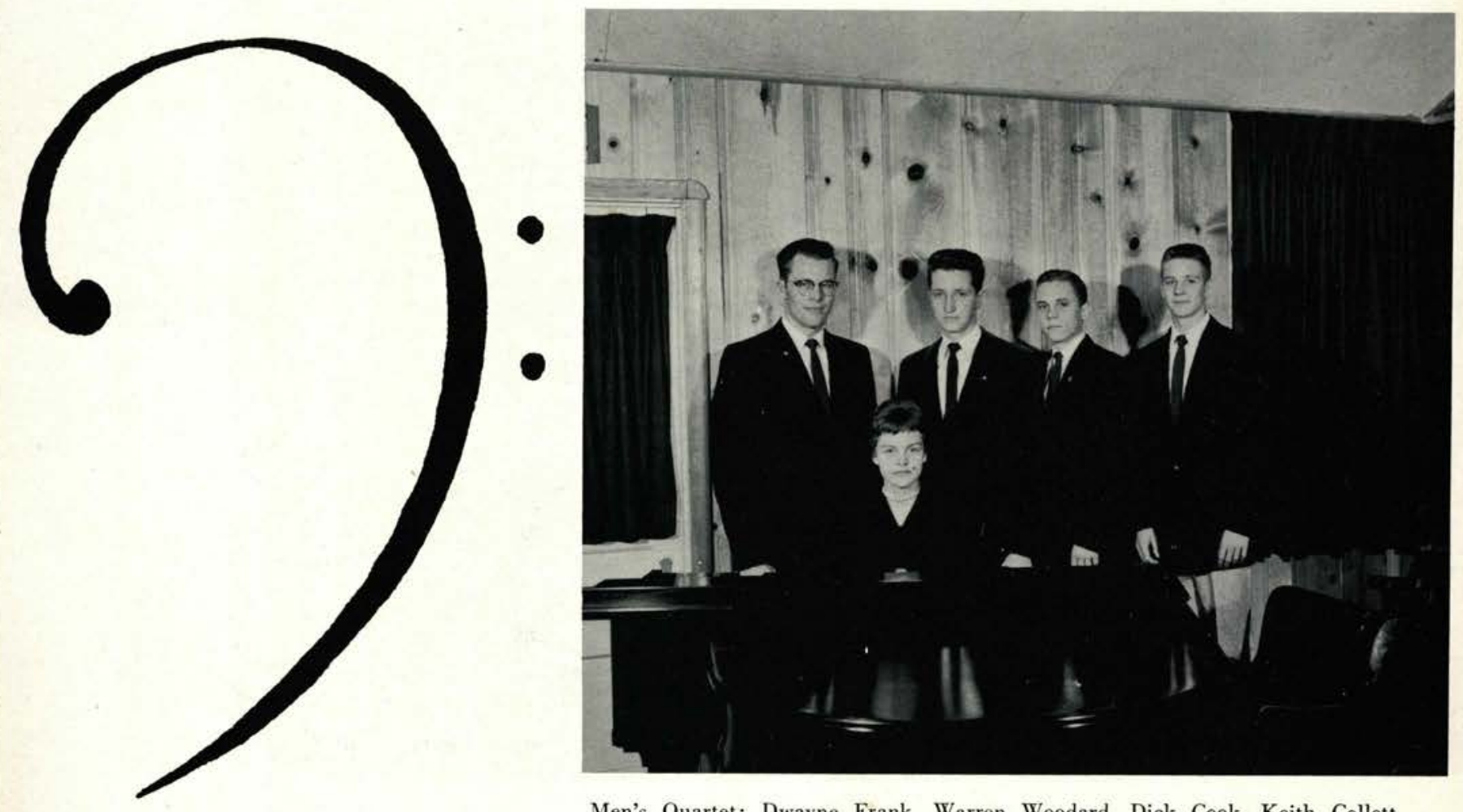

Men's Quartet: Dwayne Frank, Warren Woodard, Dick Cook, Keith Collett. Accompanist: Ruth Smelser. 

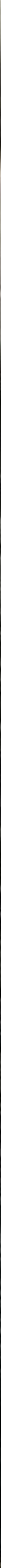

Merlin Ager

Dave Matson

Ruth Smelser

Grace Willetts
"The Music Masters Society is an honorary organization to recognize outstanding musical ability and service to the Music Department."
Carolyn Hale

Jerry Thornton

Carol Zoellner

Dwayne Frank 


\section{Suapshot Contest}

\section{Second Prize, John Entner}
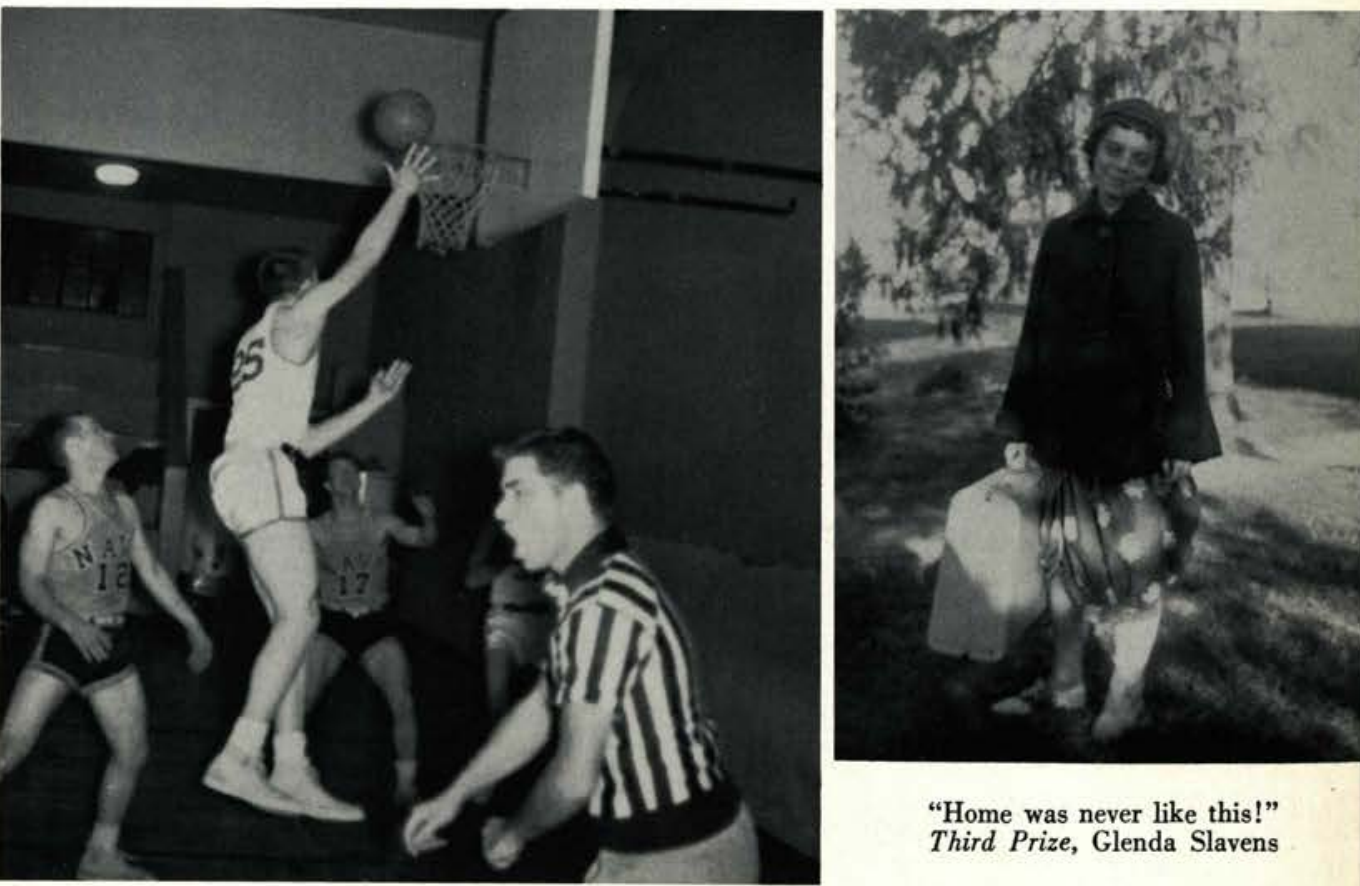

"Someday you'll be a Senior!"

First Prize, Joyce Grant

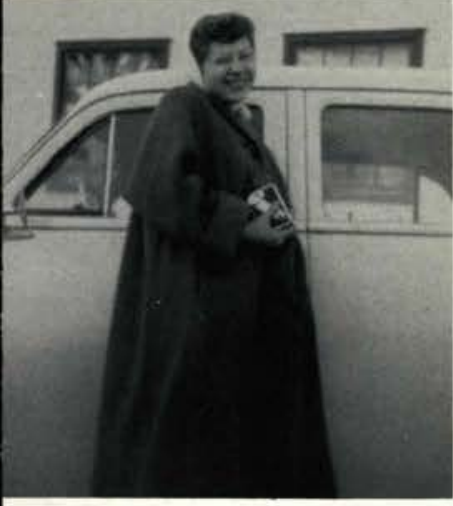

"Cutie!"

"Uncle Sam will teach you how, Bob!"

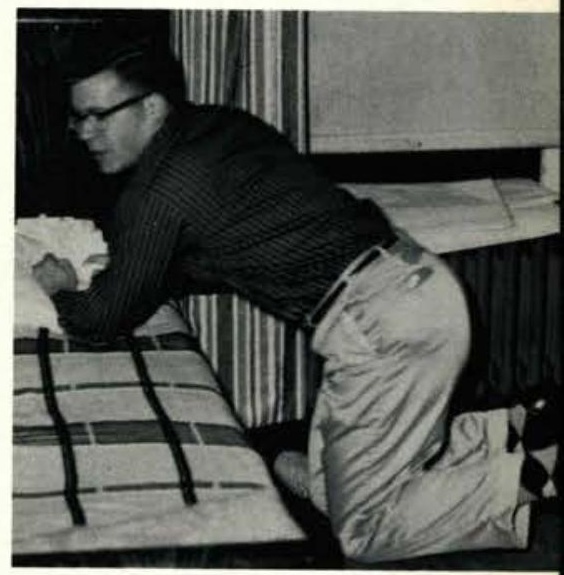

"Home was never like this!" Third Prize, Glenda Slavens
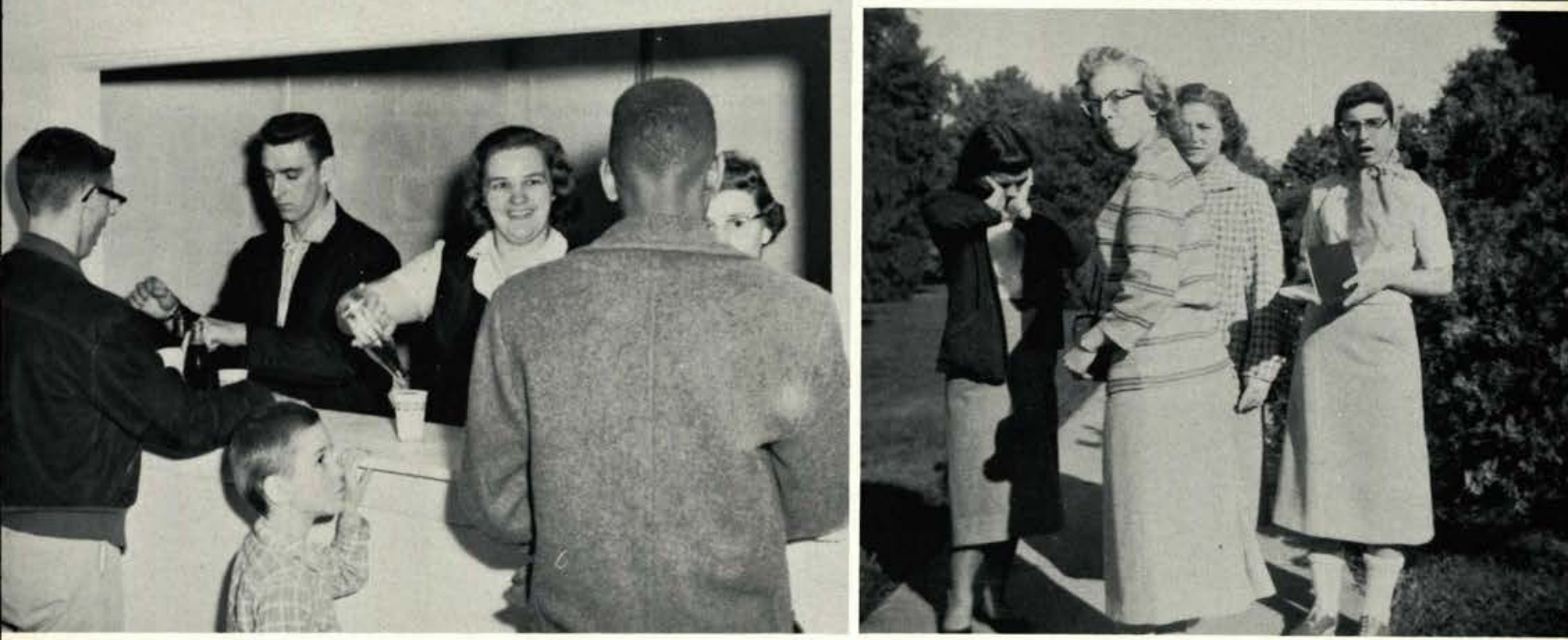


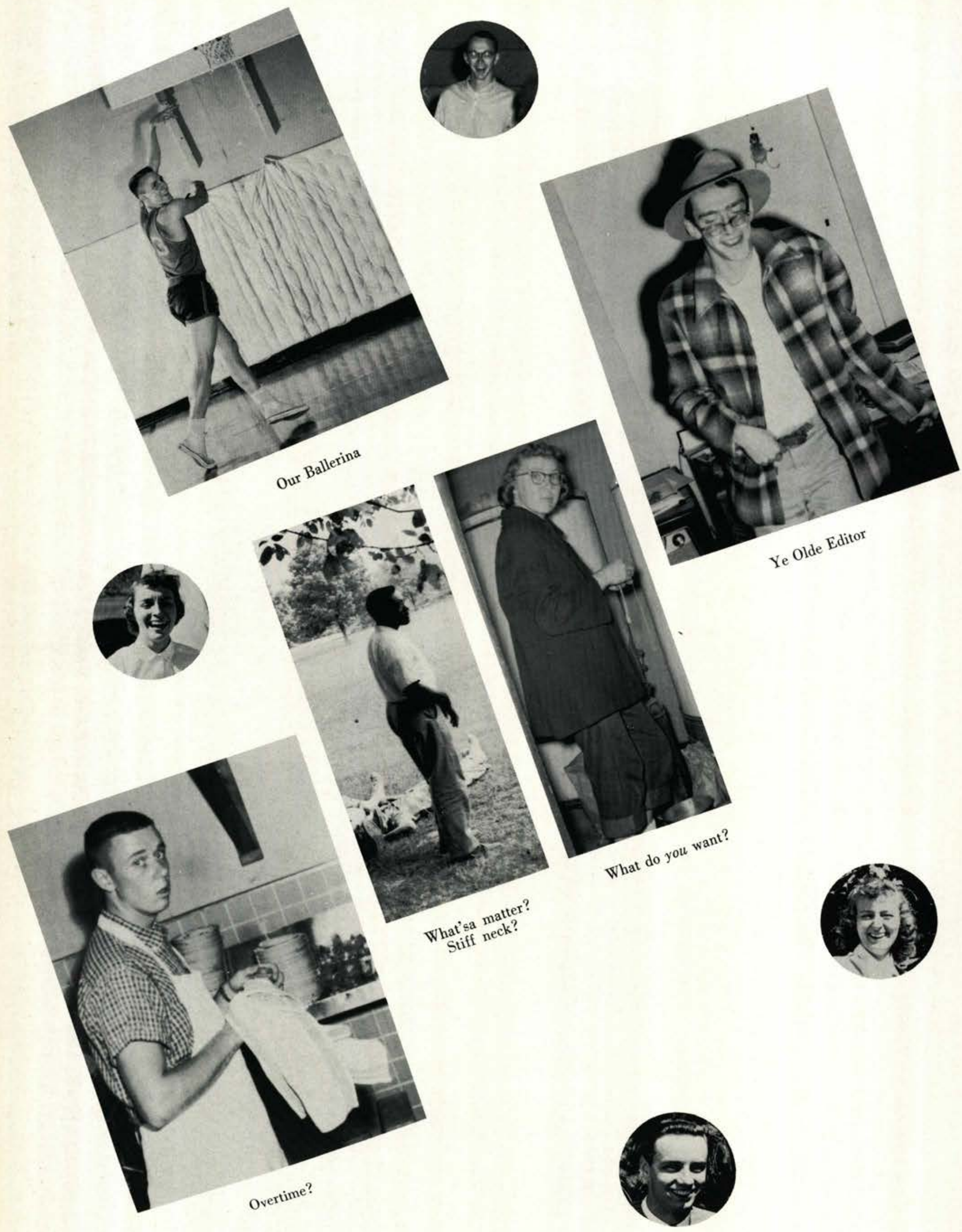




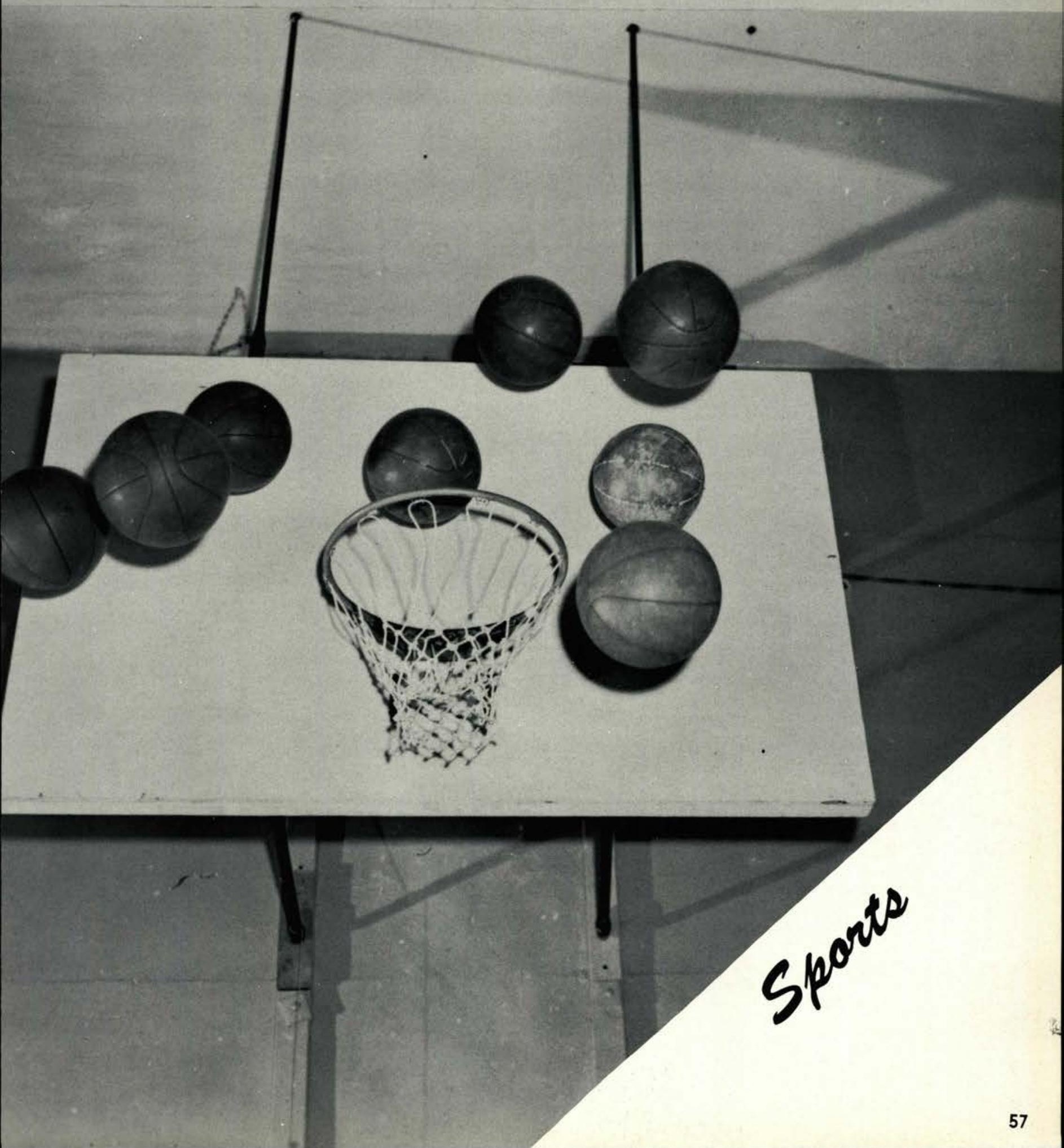




\section{The \\ Coach}

COACH BOSWER
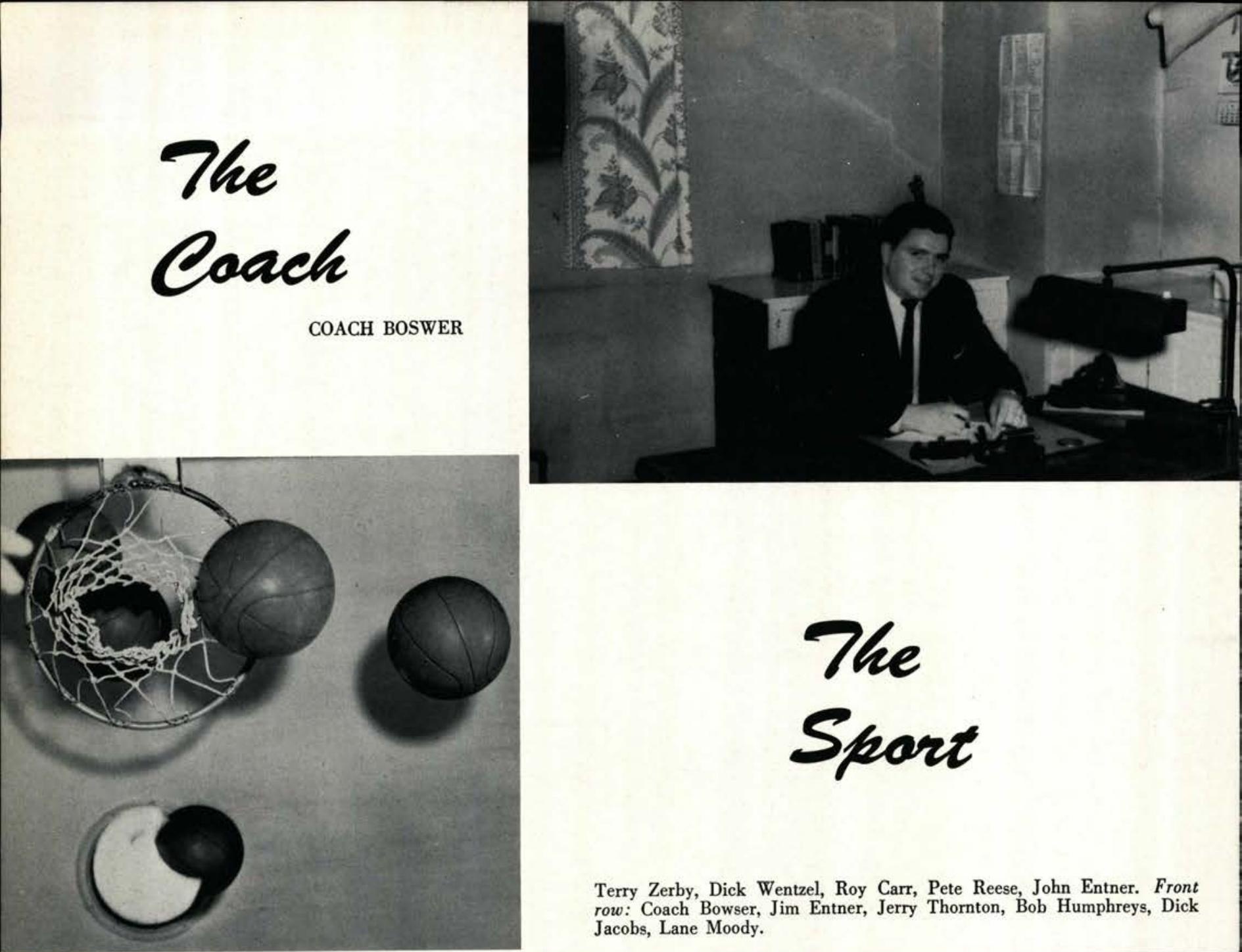

\section{The \\ Spart}

Terry Zerby, Dick Wentzel, Roy Carr, Pete Reese, John Entner. Front row: Coach Bowser, Jim Entner, Jerry Thornton, Bob Humphreys, Dick Jacobs, Lane Moody.

\section{The \\ Team}

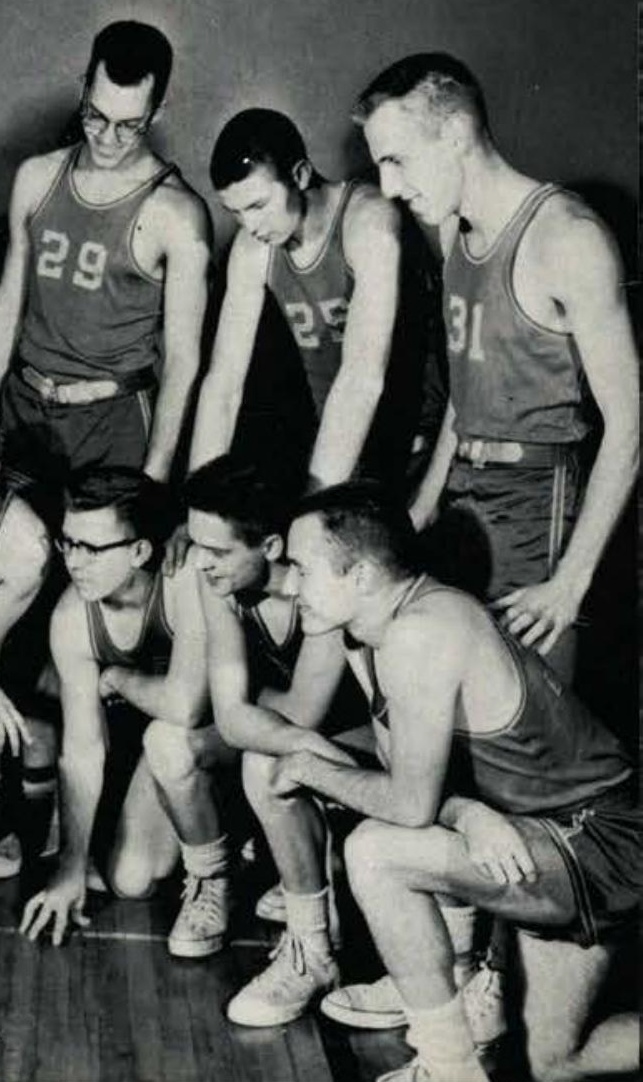


10

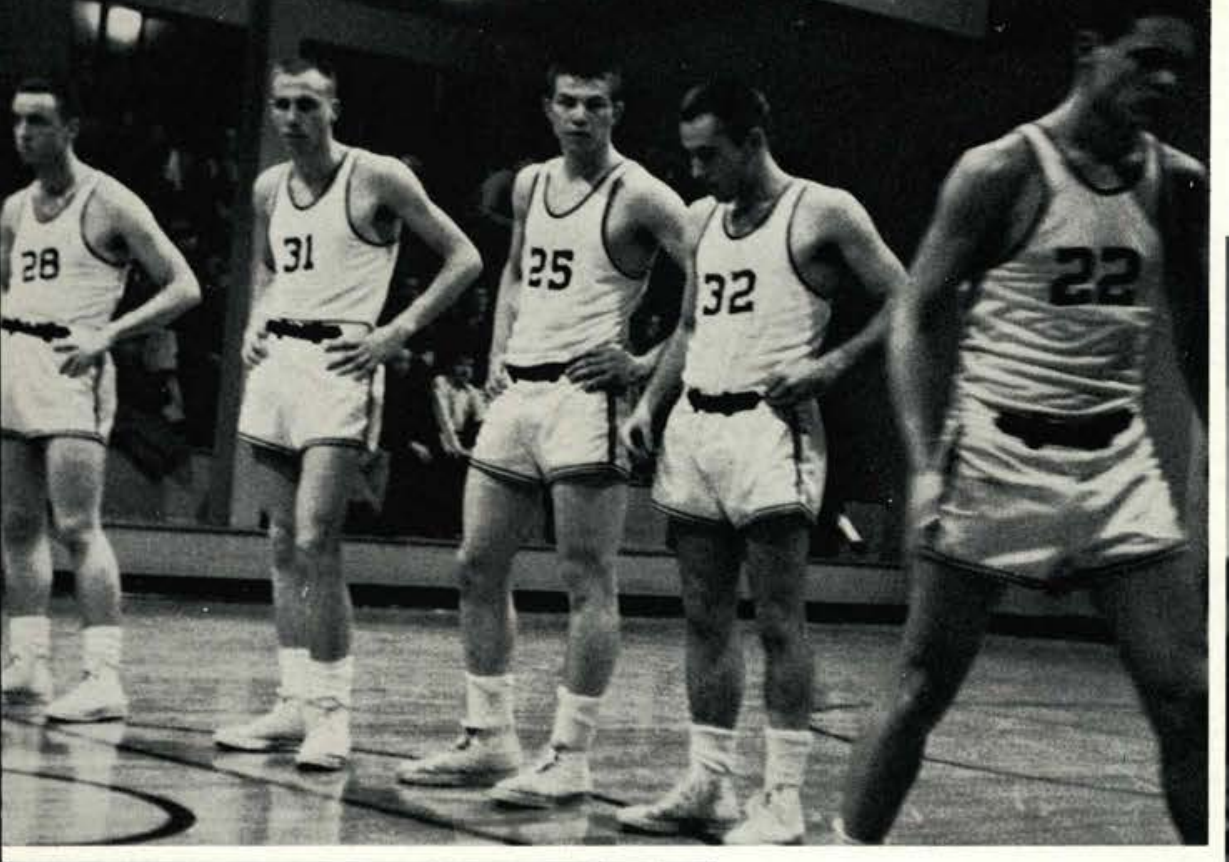




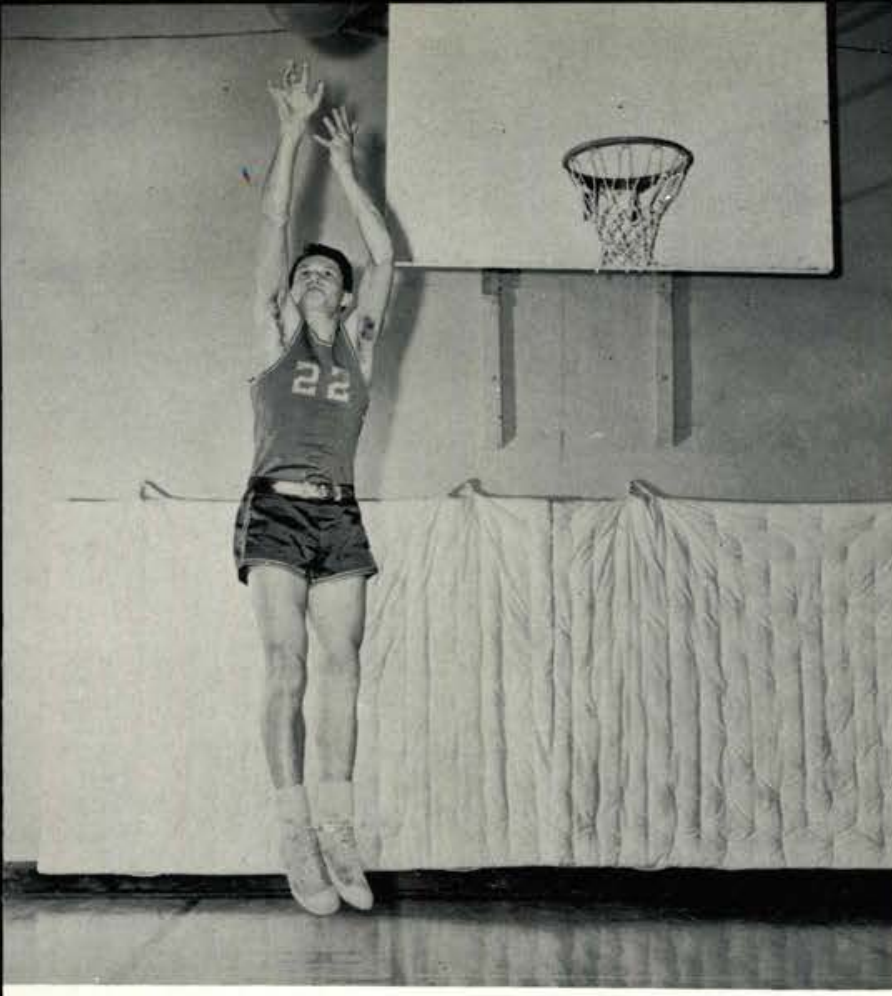

DICK JACOBS

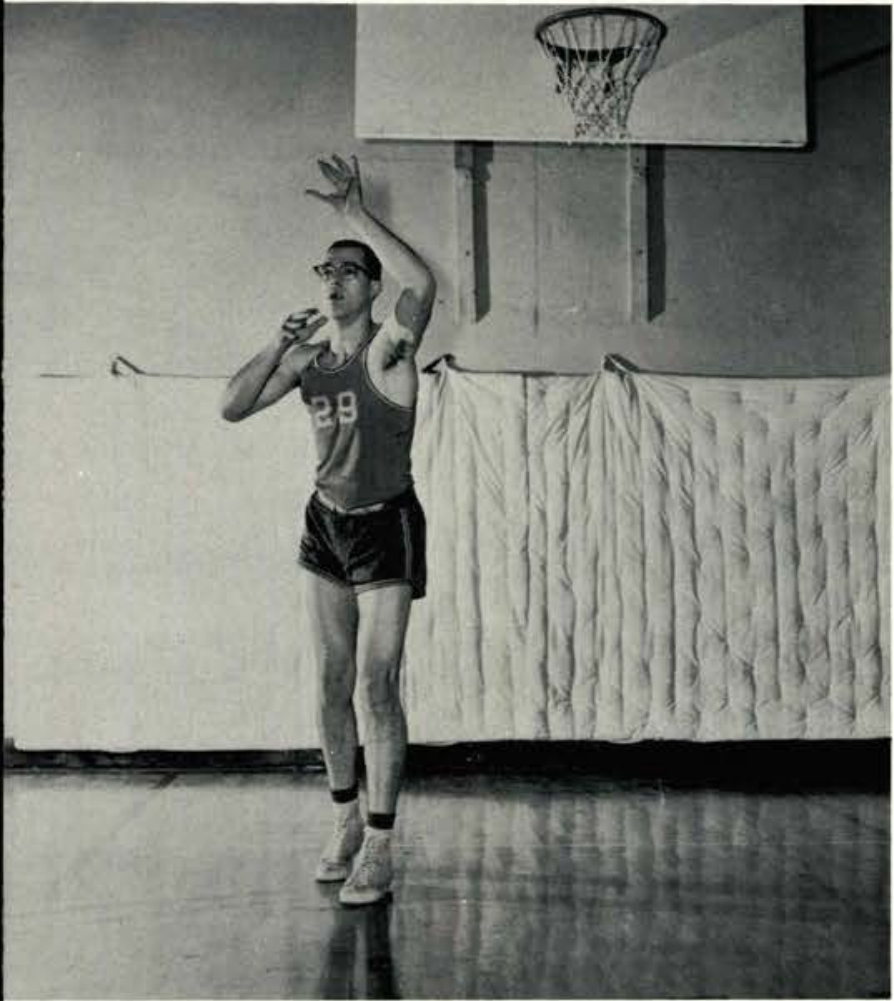

ROY CARR

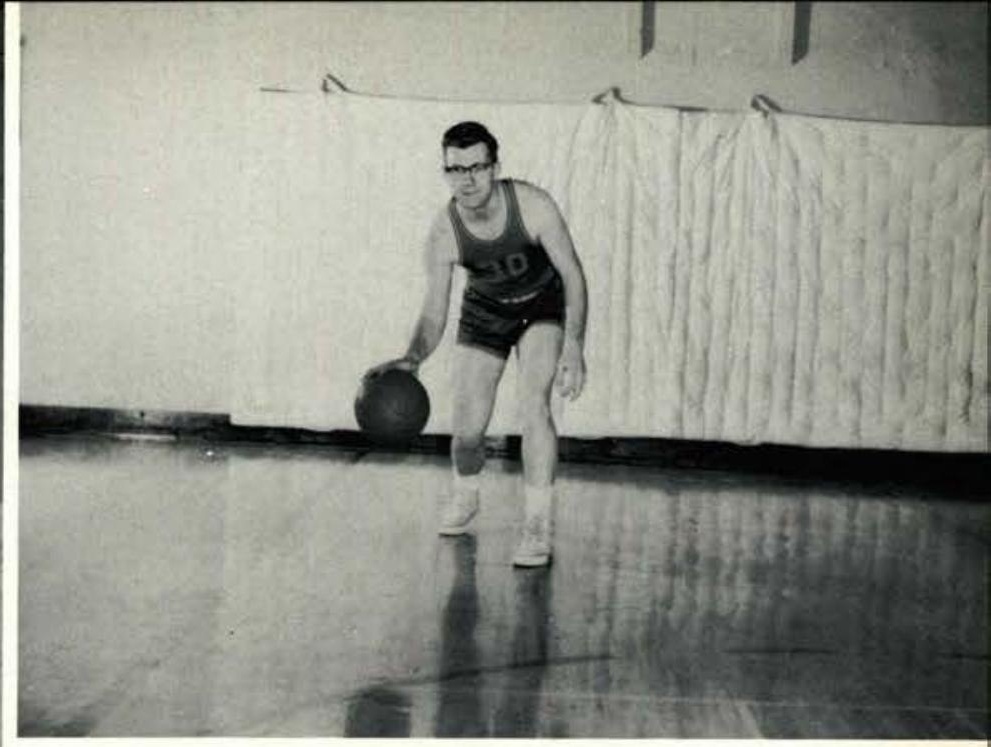

BOB HUMPHREYS

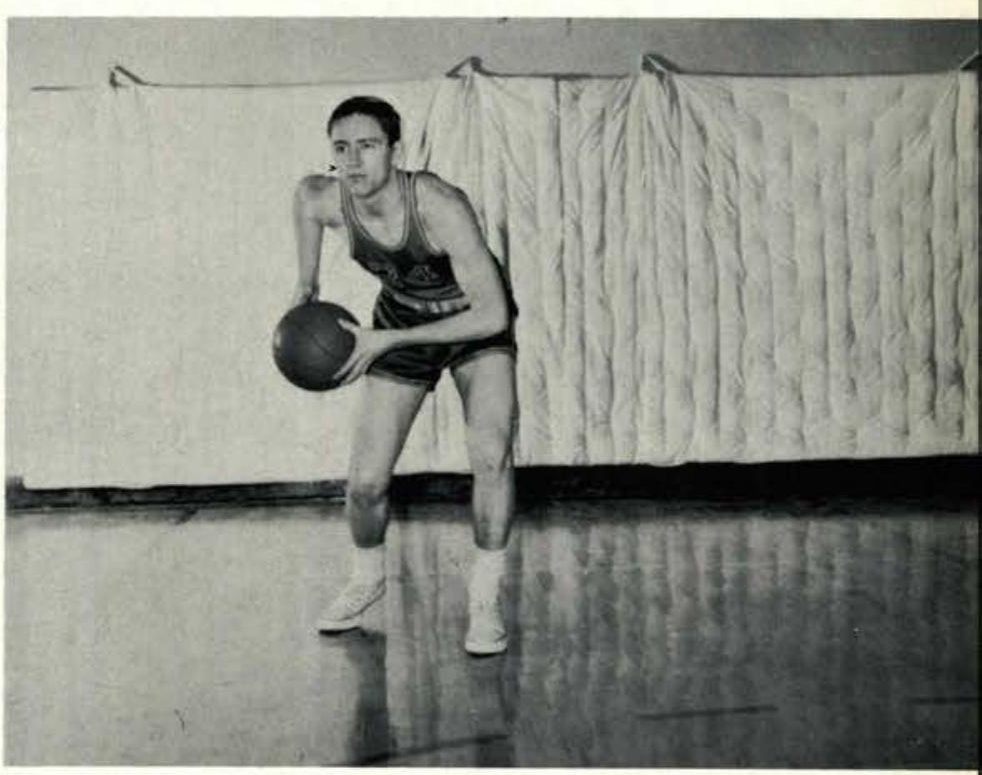

TERRY ZERBY

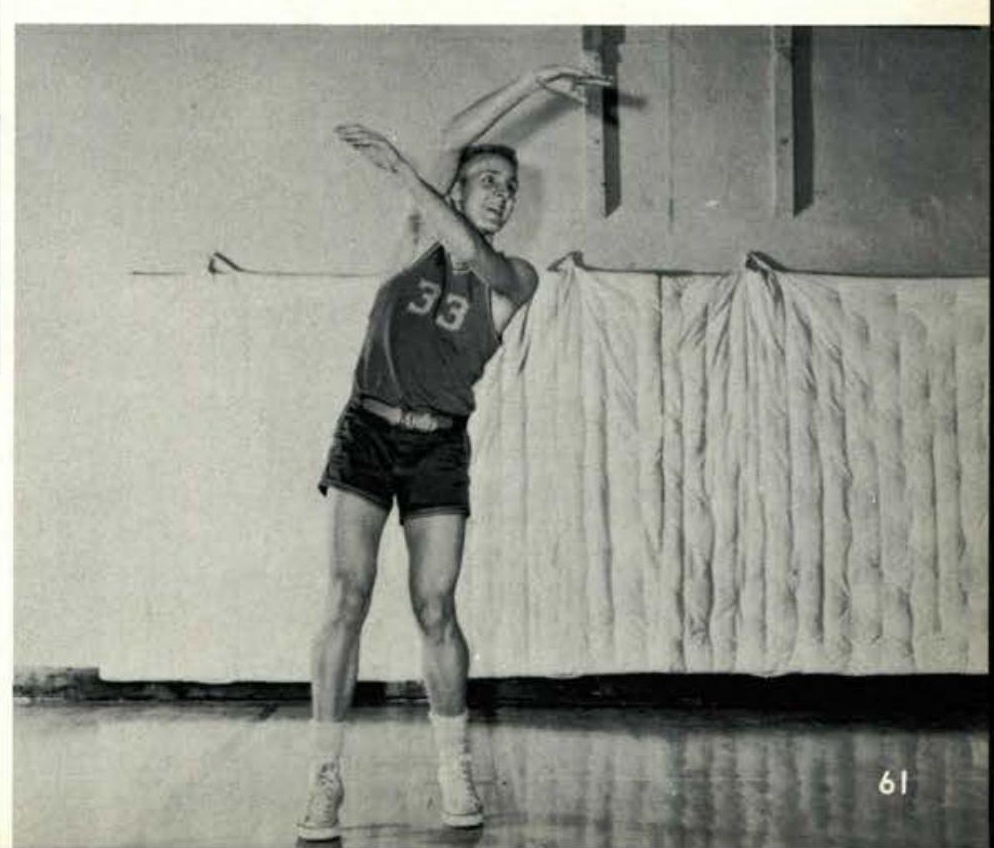




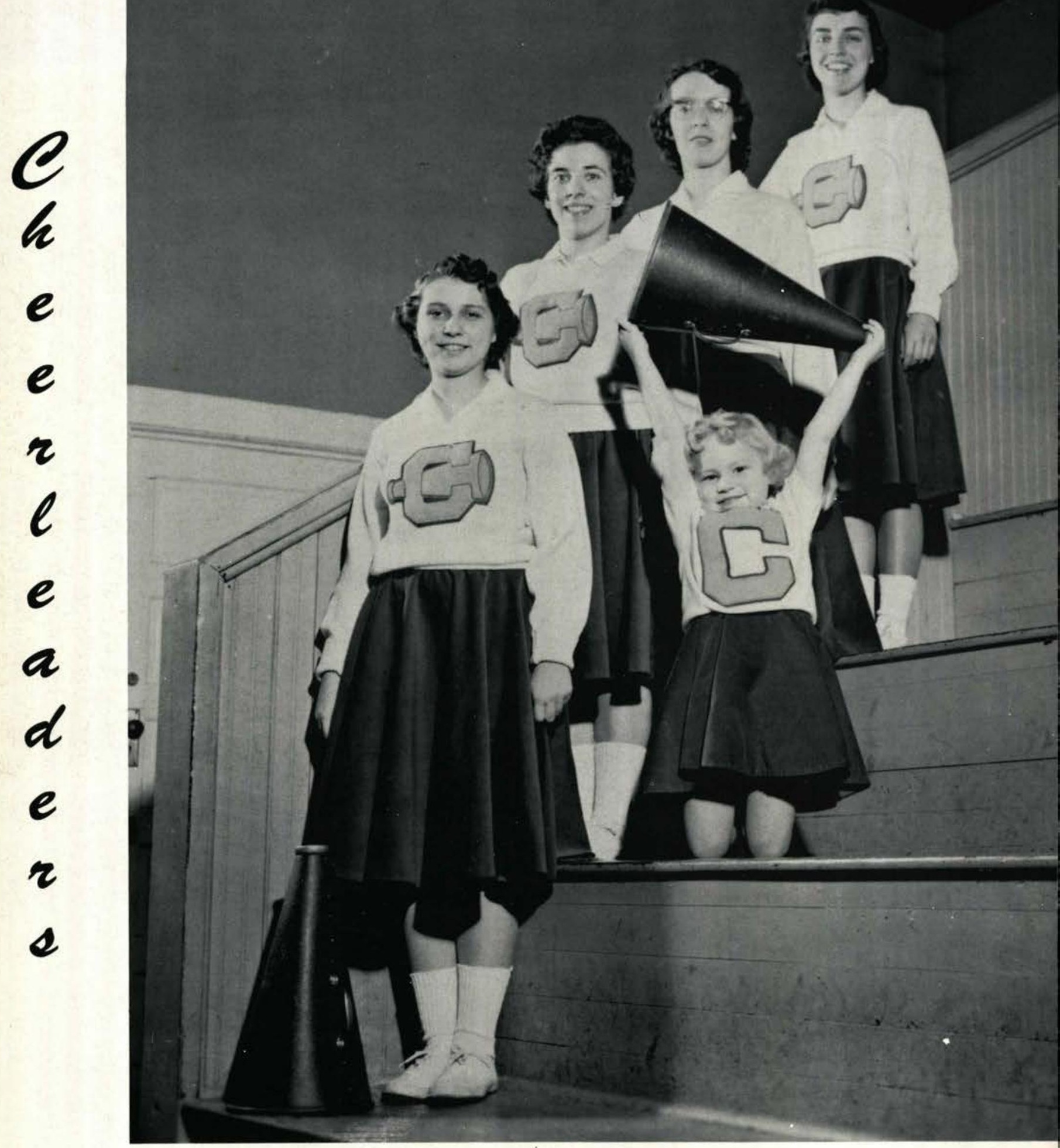

Marlene Davis, Sandy Millikin, Captain, Rosemary Smith, Esther Weiss, Jayne Ann Wentzel. 


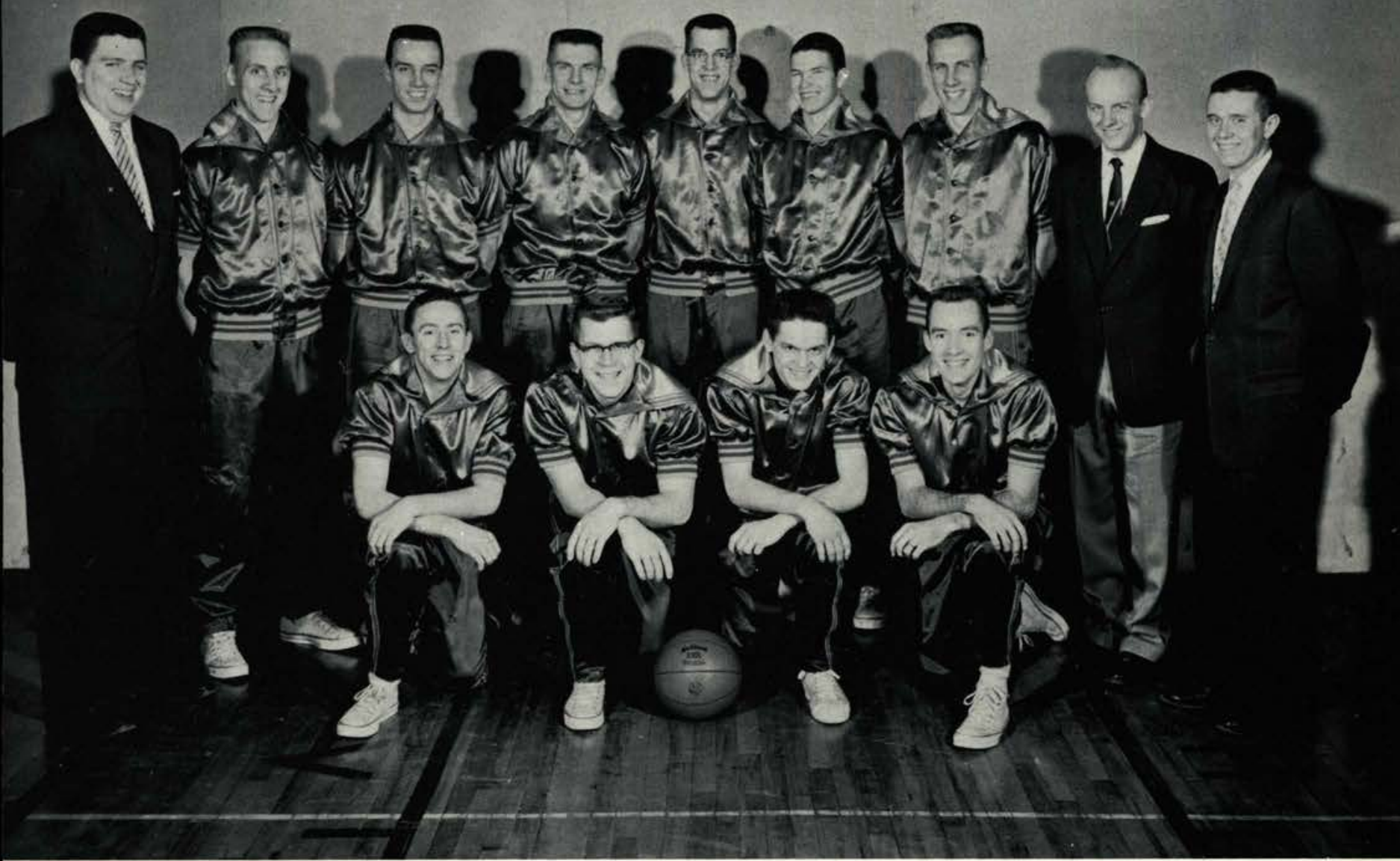

Back row: Coach Bowser, Jim Entner, Jerry Thornton, Dick Wentzel, Roy Carr, Pete Reese, John Entner, Dave Thomas, Manager; Les Webster, Statistician. Front row: Terry Zerby, Bob Humphreys, Dick Jacobs, Lane Moody.

\section{Varsity}

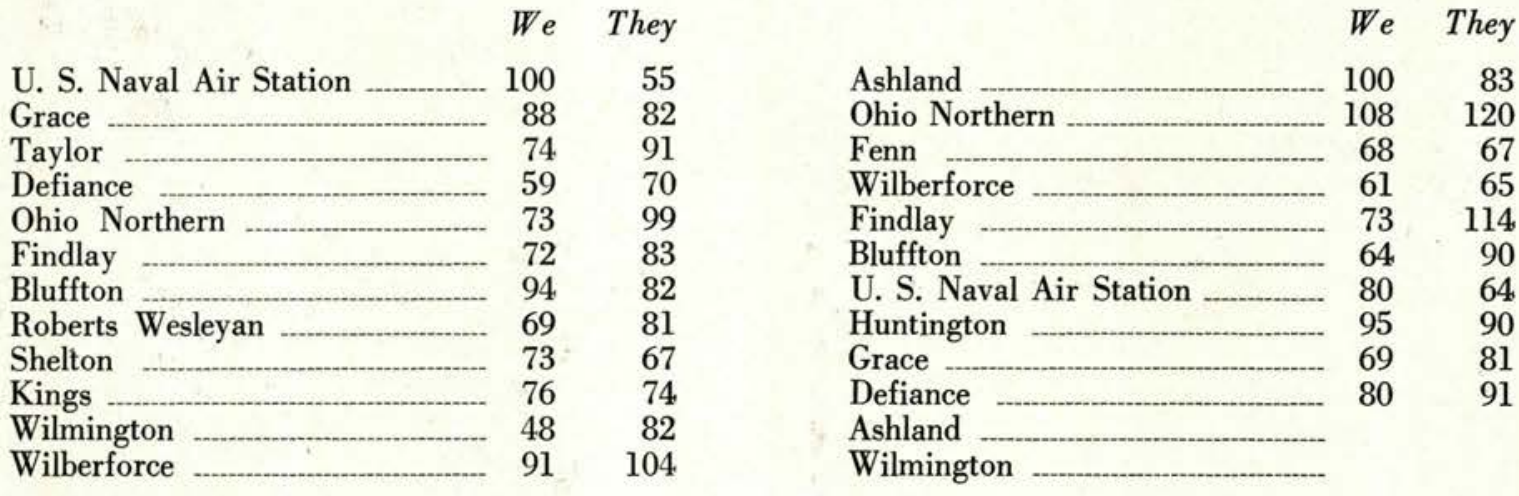




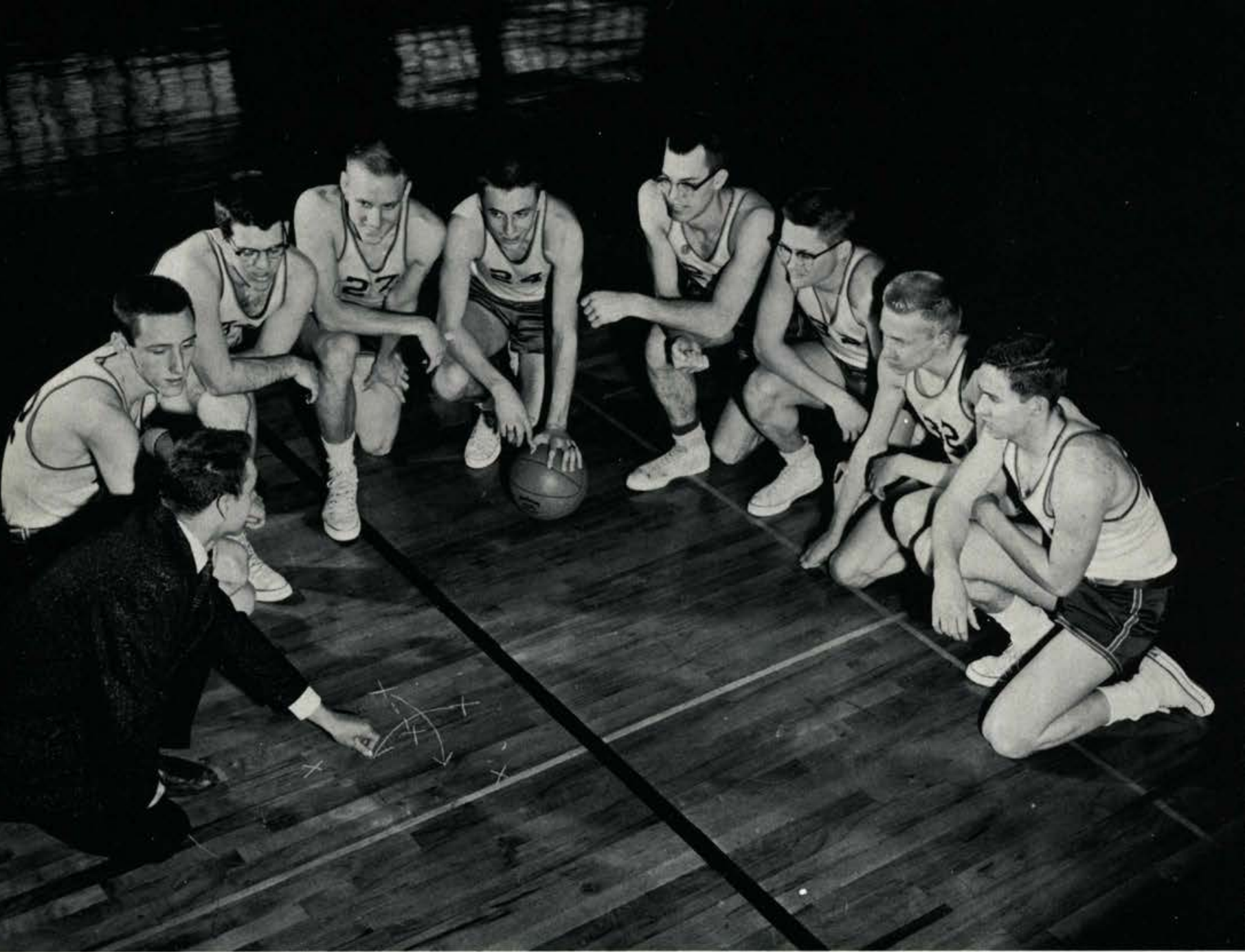

Bob Marcellino, Coach; Bob Howder, Dave Dautel, Jim Entner, Bob Self, Roy Carr, Dick Graven, Dan Purdy, Terry Zerby.

\section{Guniar Varsity}

We They

We They

Goodson Brothers

$62 \quad 68$

68

Ashland

45

51

Miami Jacobs

71

Ohio Northern

$45 \quad 61$

Findlay

50

63

Bluffton

66

71

Wilmington

60

62

Ohio Northern

$51 \quad 88$

Columbus

$73 \quad 38$

Dayton _-_ _ _ _ _ _ _ - $76 \quad 60$

Goodson Brothers …………......... $51 \quad 56$

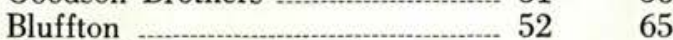

Dayton Emmanuel Baptist _........... $72 \quad 55$

Grace _...__ $66 \quad 46$

Defiance ___ _ _ _ _ $54 \quad 57$

Dayton Emmanuel Baptist ............ 64.51 


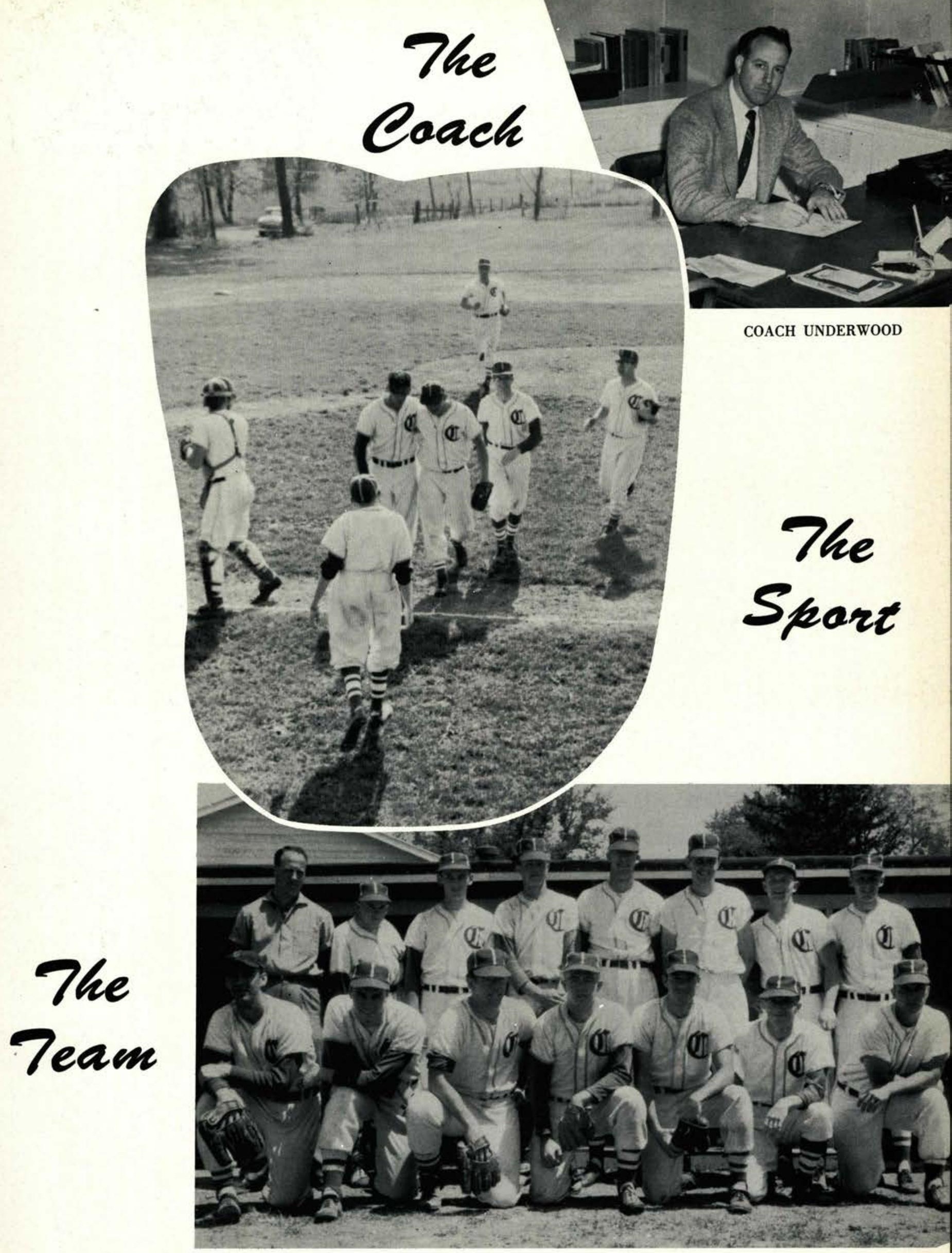

Back row: Coach Underwood, J. Willetts, M. Ager, P. Reese, R. Carr, W. Wentzel, A. Griffin,

D. Thomas. Front row: B. Marcellino, J. Thornton, D. Dautel, R. Erickson, L. Moody, W. Capron,

J. Hartman. 


\section{Action}

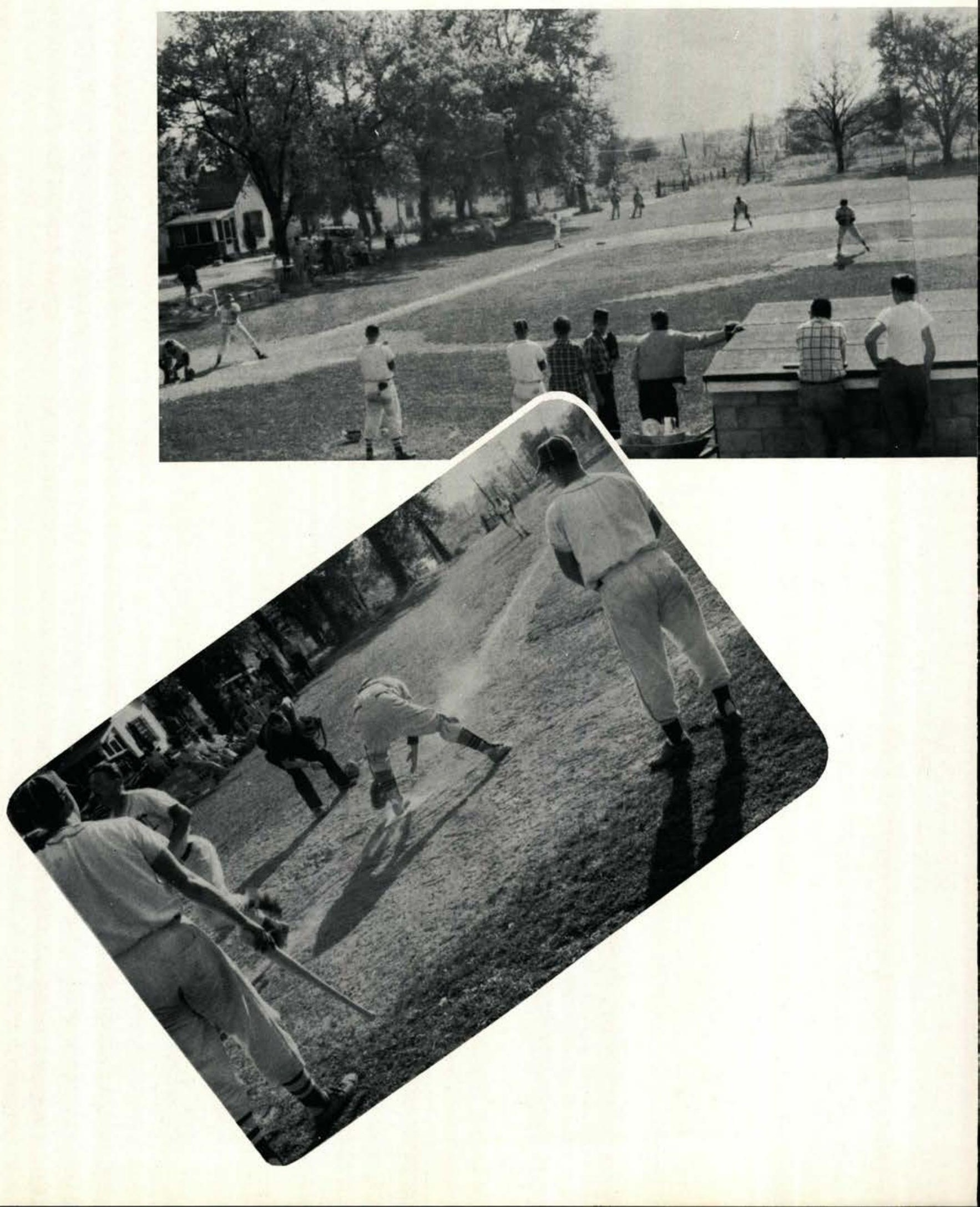




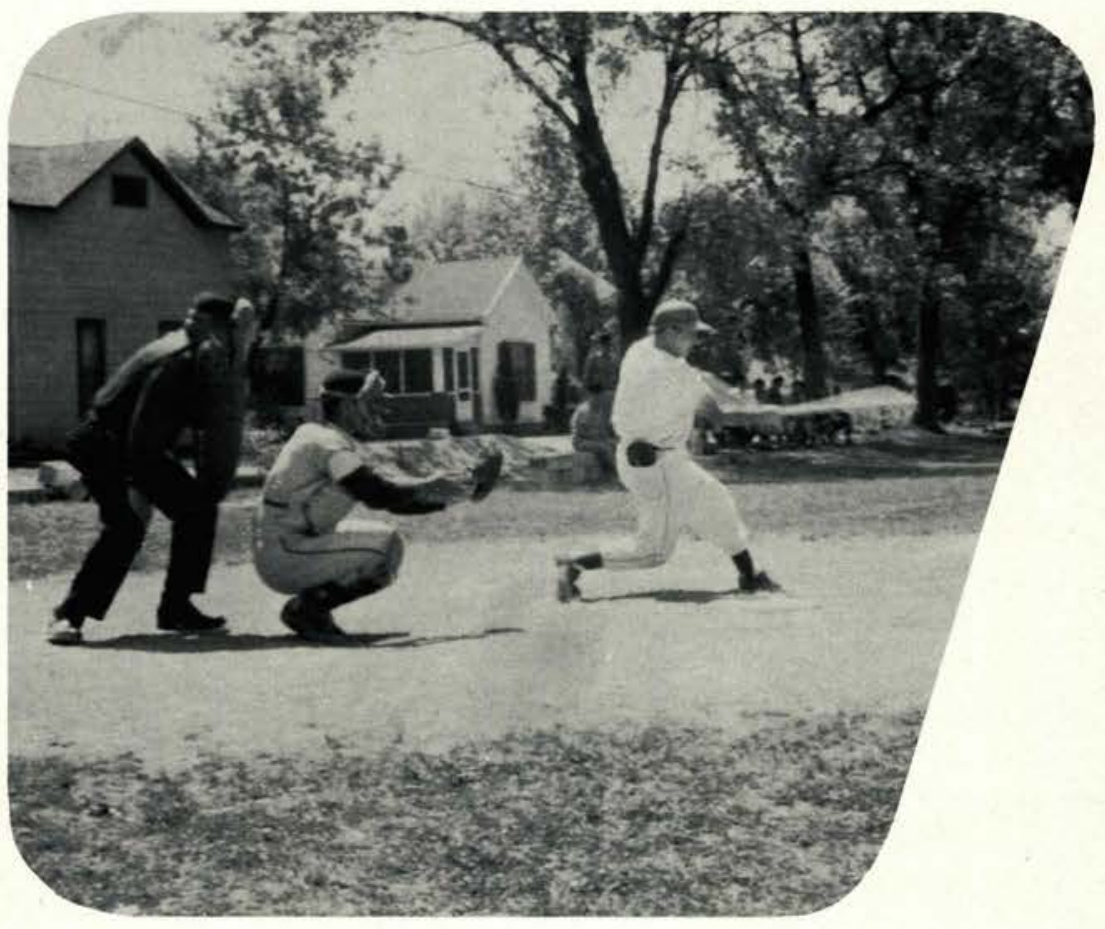




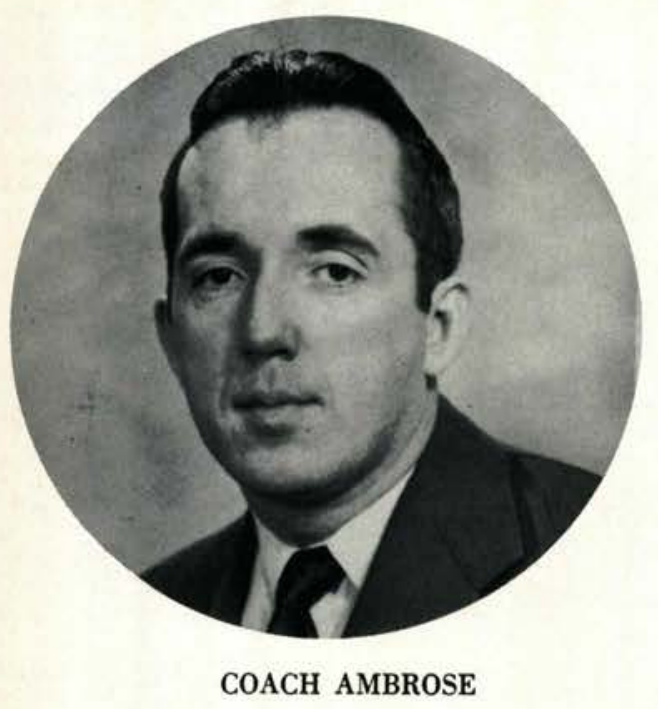

\section{The Coach}
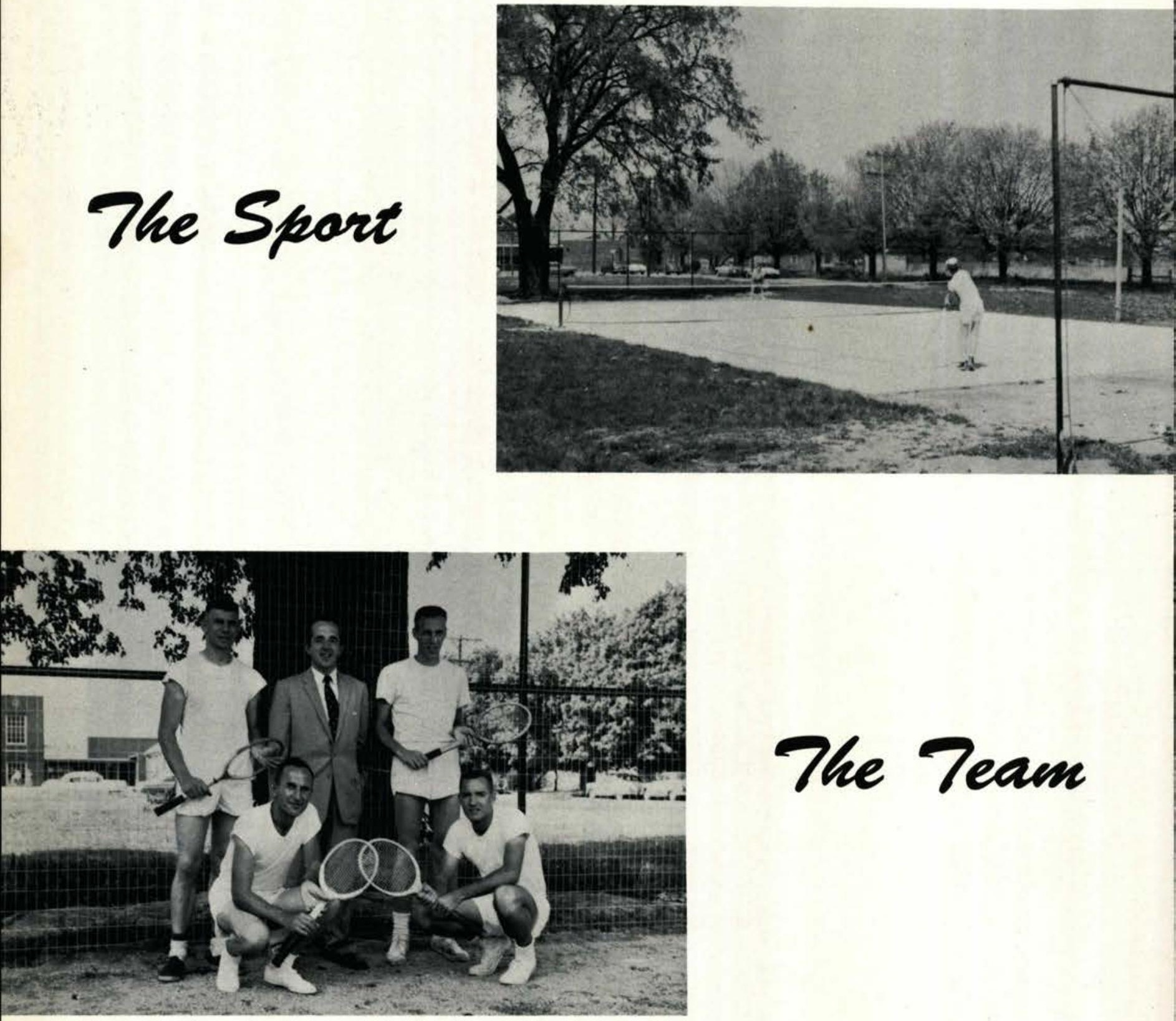

The Team

Dick Wentzel, Coach Ambrose, John Entner, Stewart Chaffe, Lane Moody. 


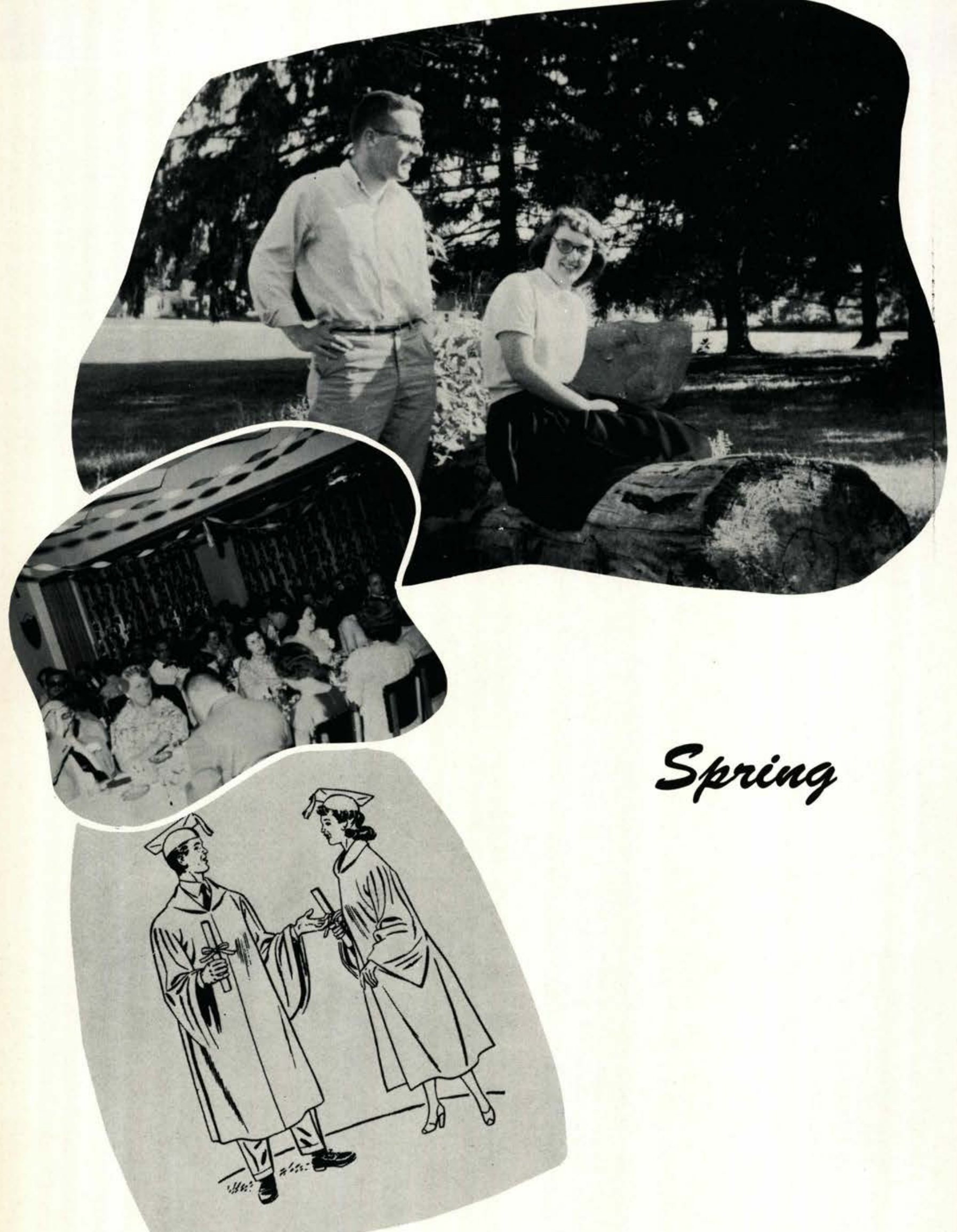




\section{Prospective}

Registration

Sacred Medley

"The Blotters"

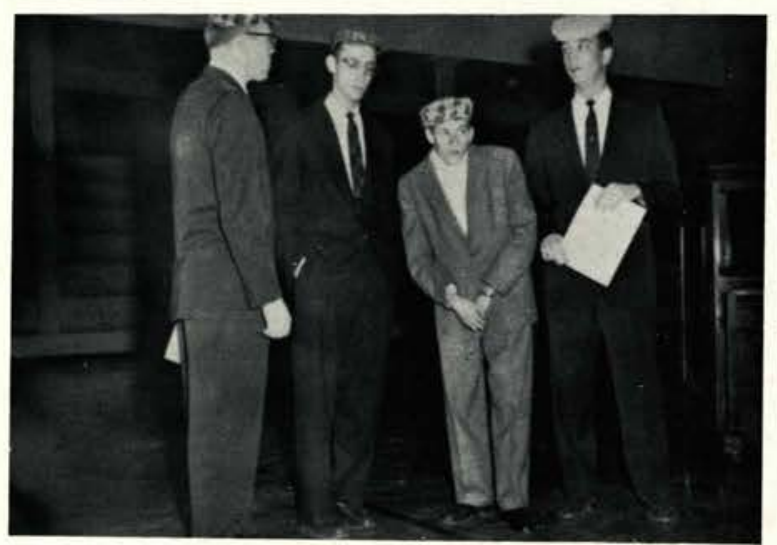

Students' Day

1

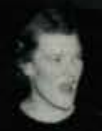




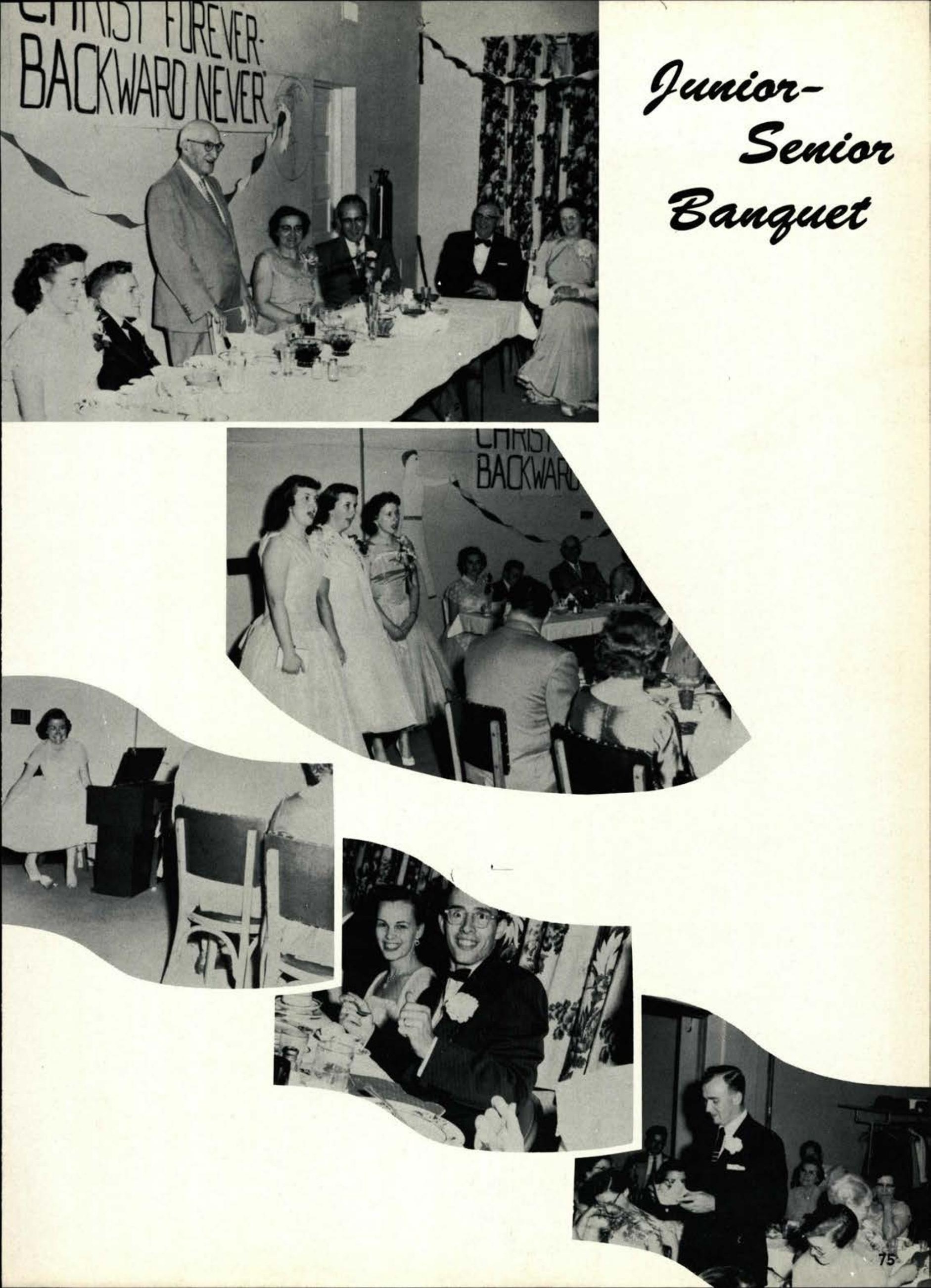




\section{Lamentations}

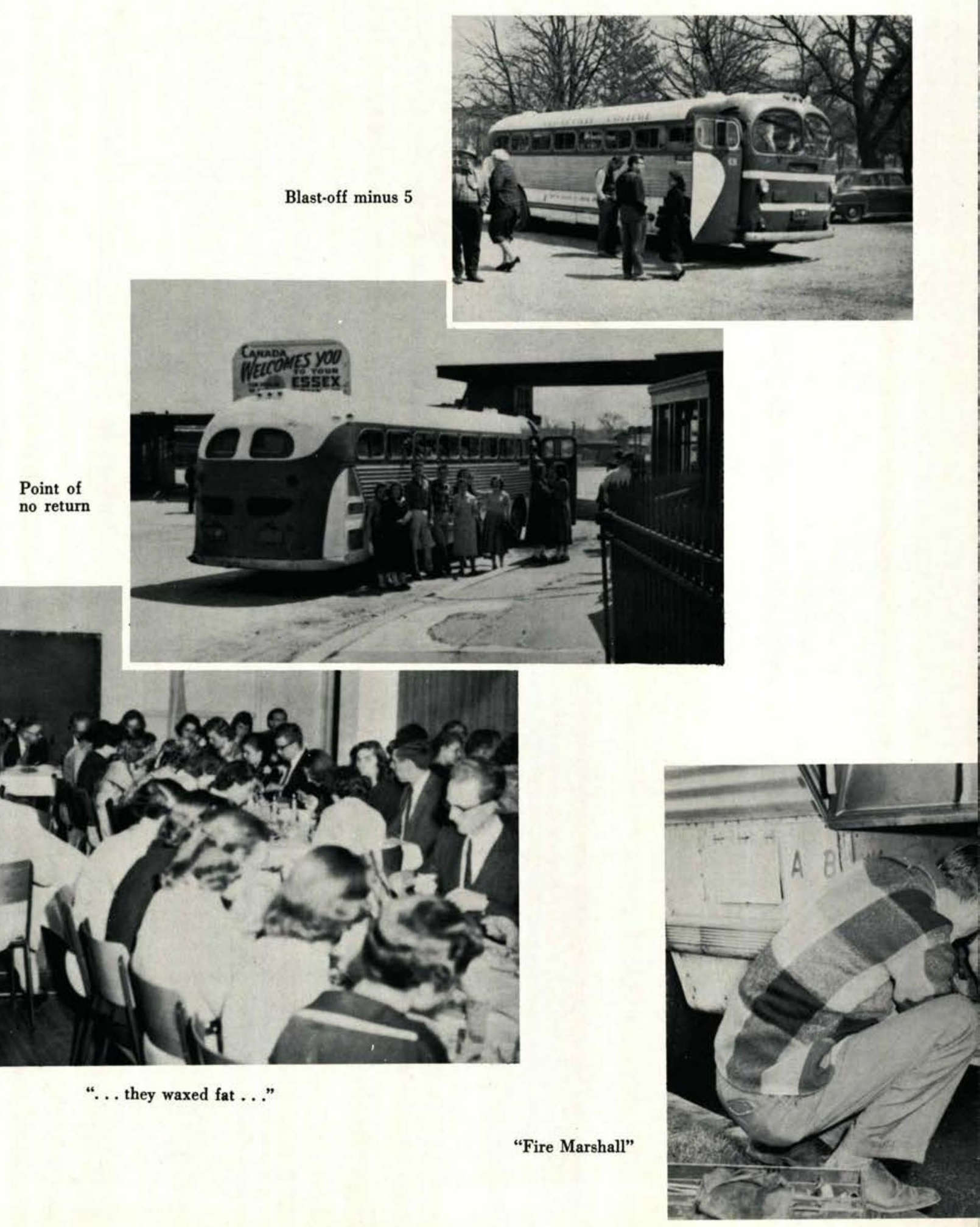




\section{of Geremial}

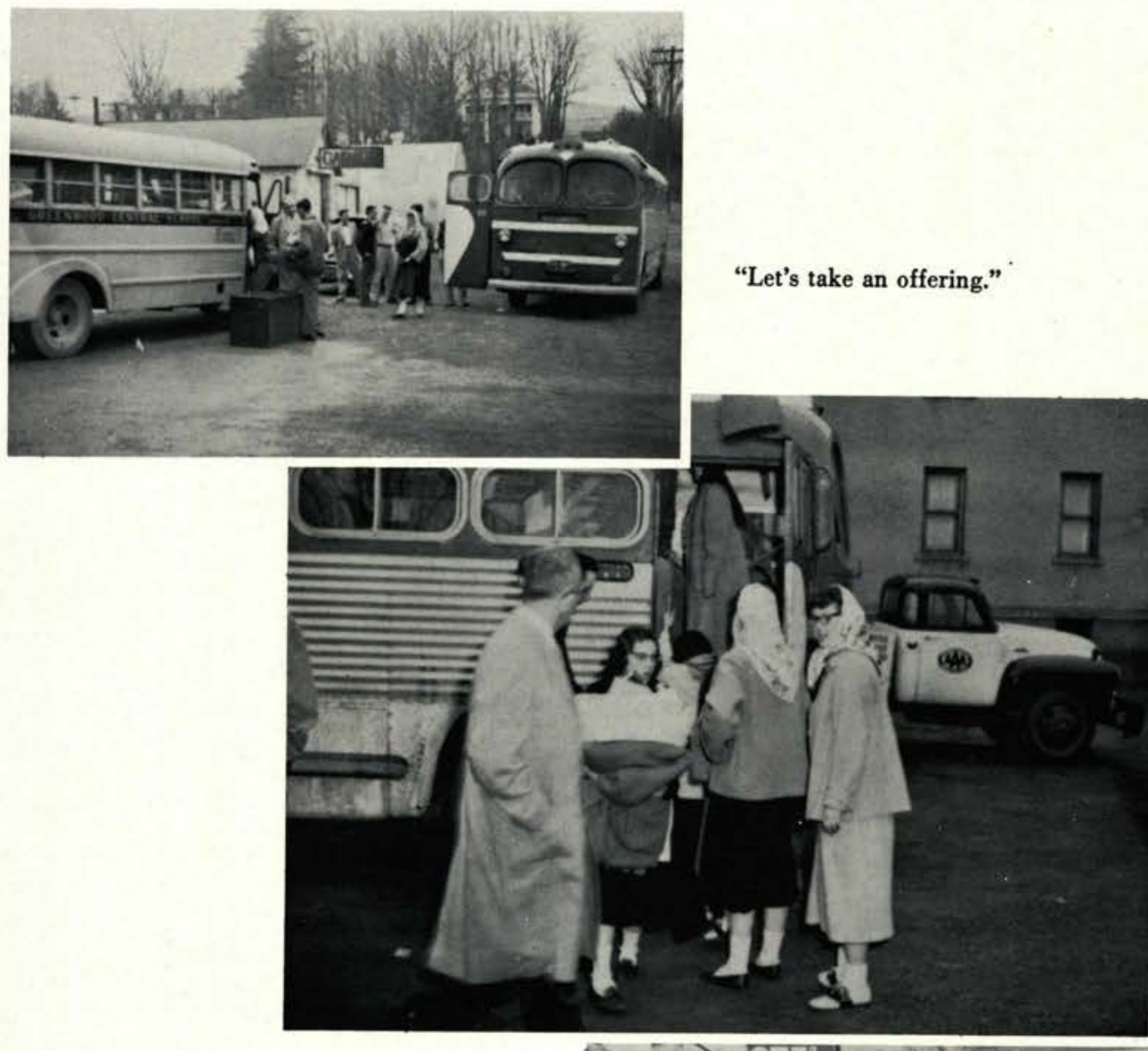

Where's the

male box?

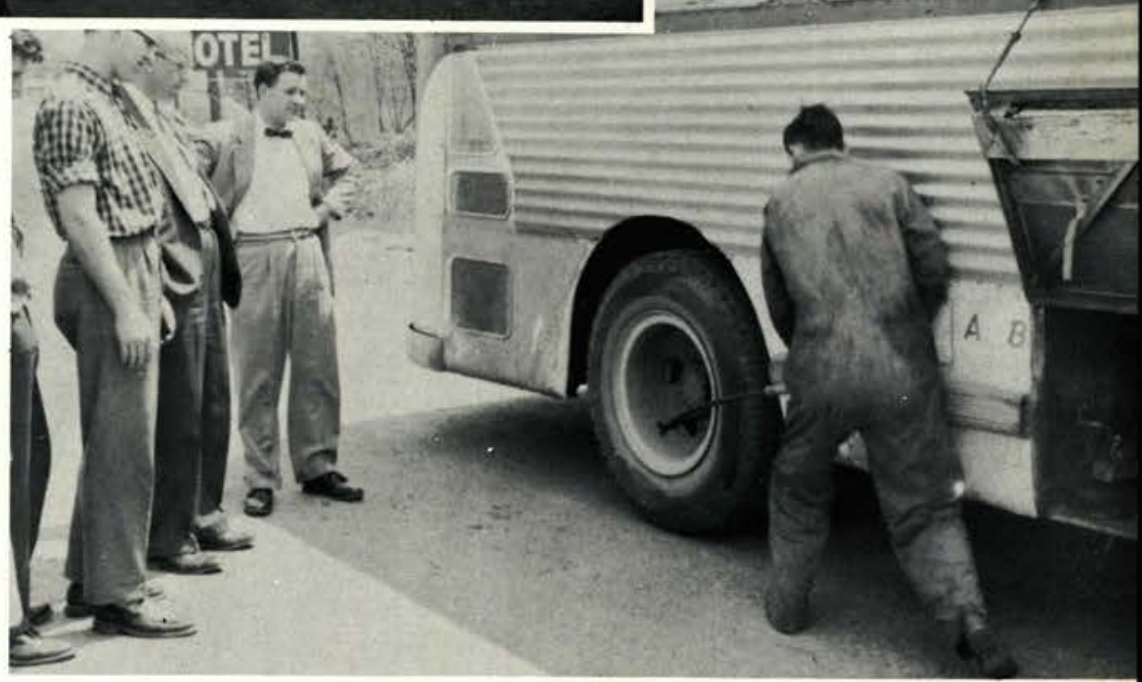

Sidewalk supervisors 


\section{Dramatis}

\section{Personae}
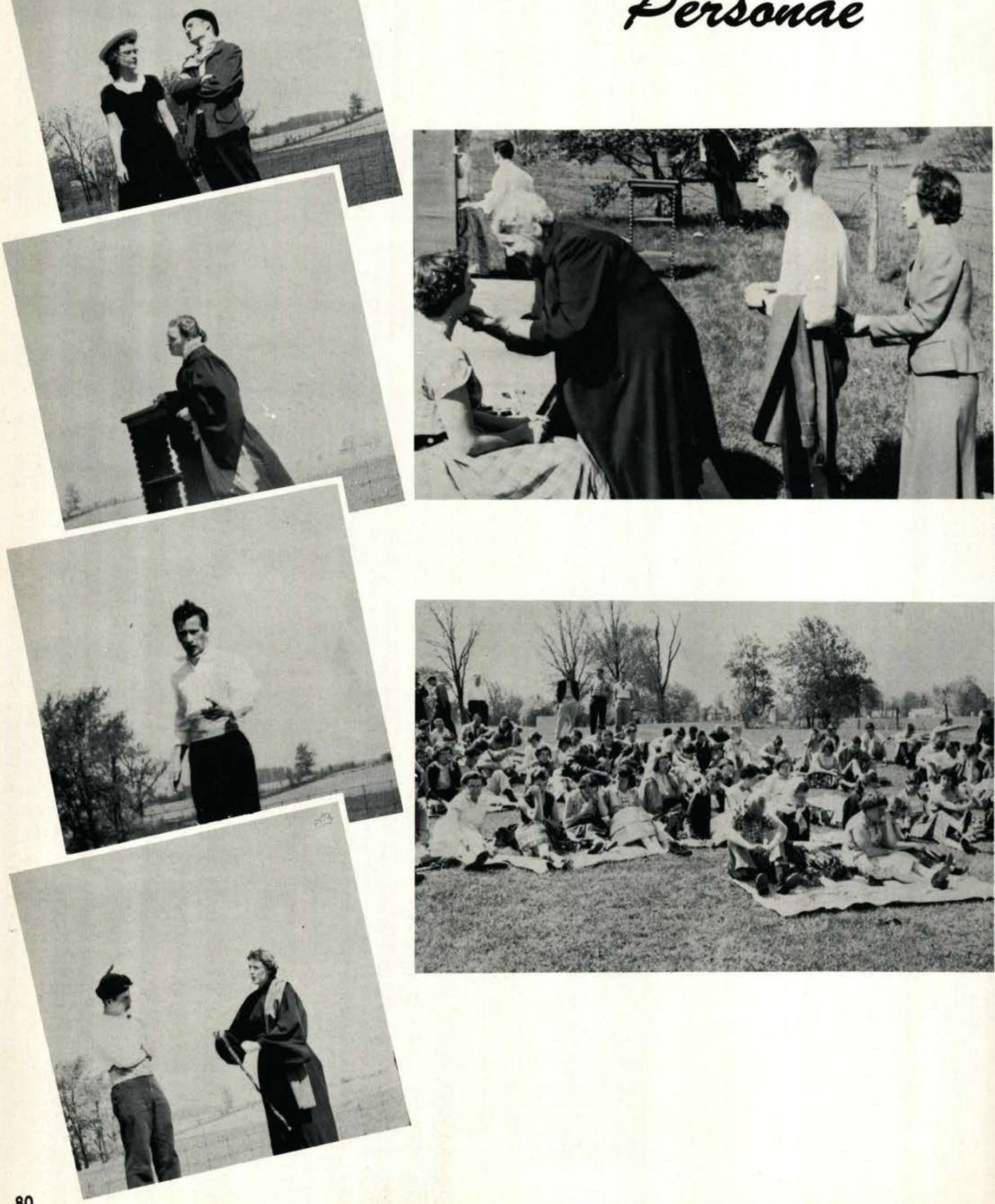

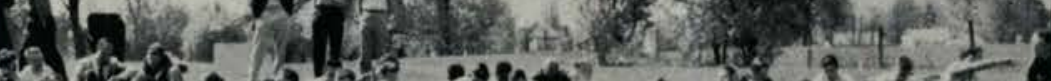

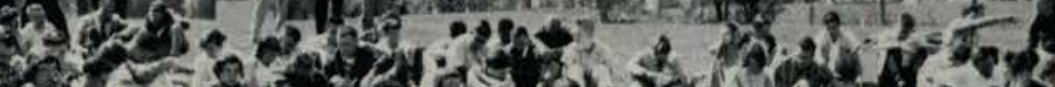

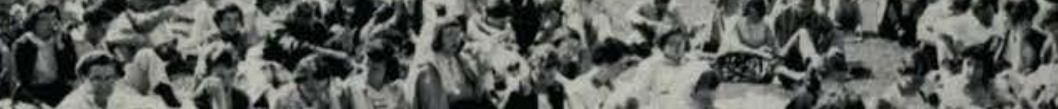

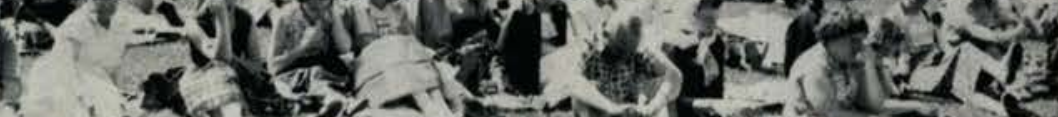

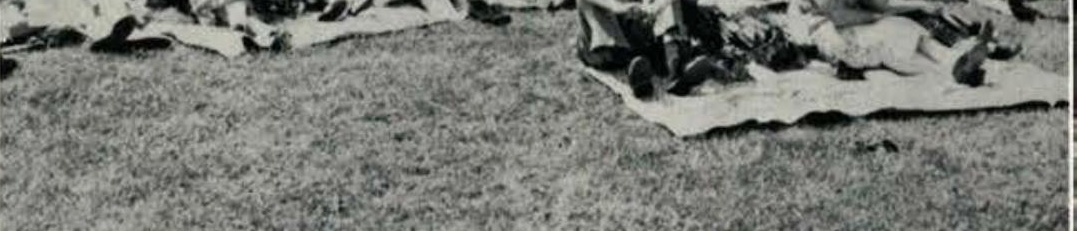




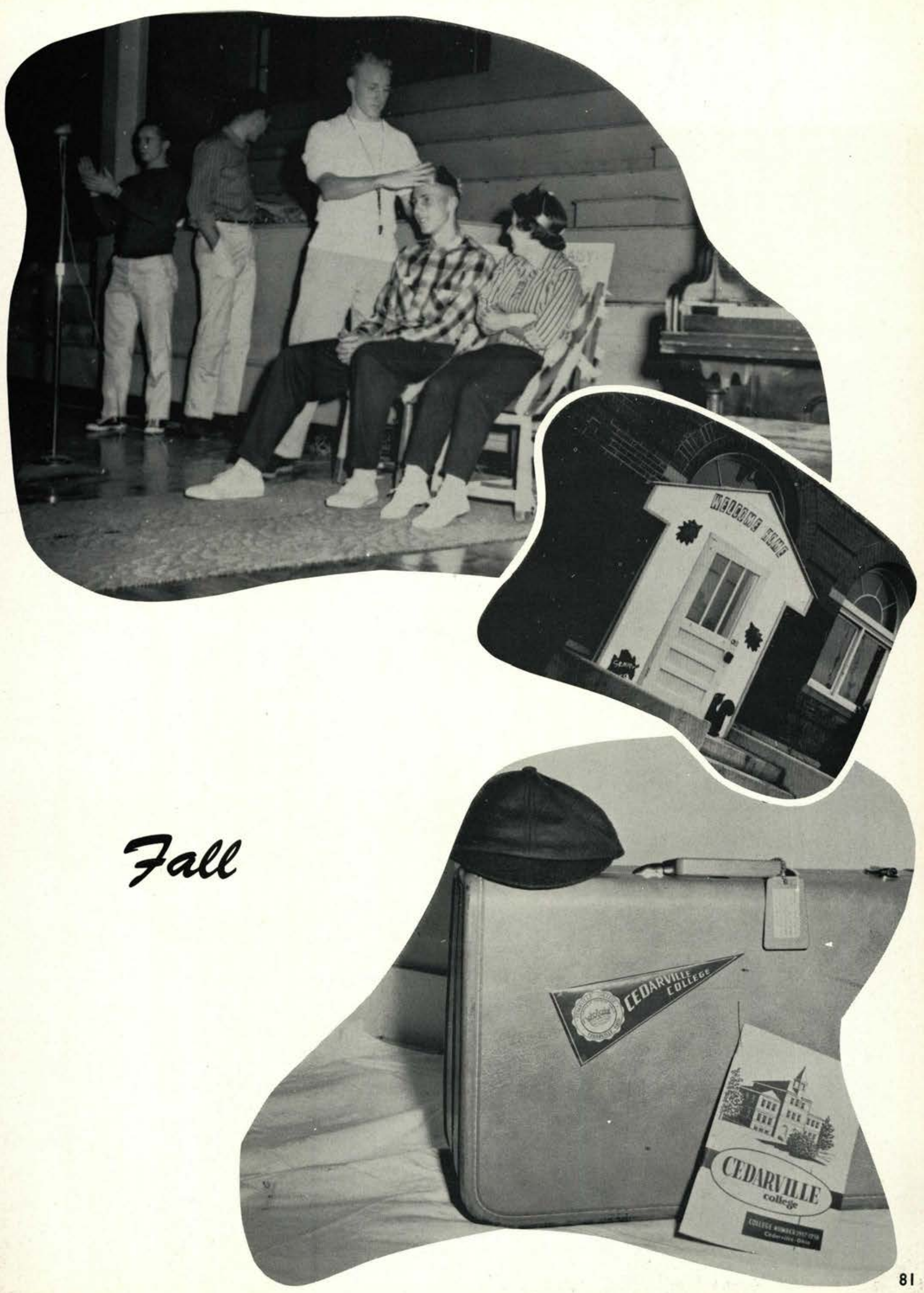




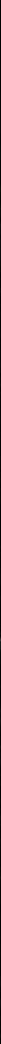

Is this a "Fay original?"

Some things I just won't eat!

\section{Freshman}

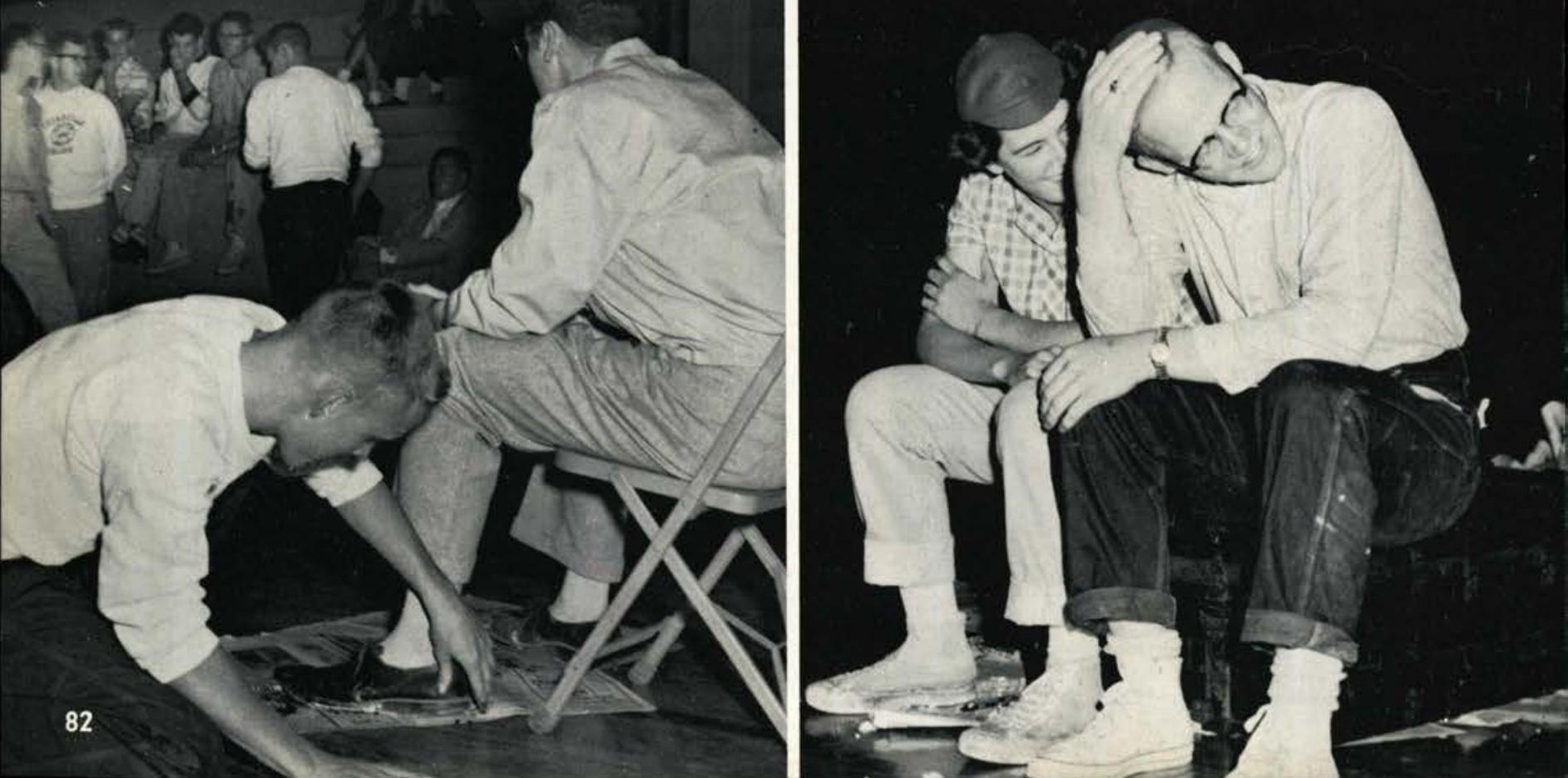




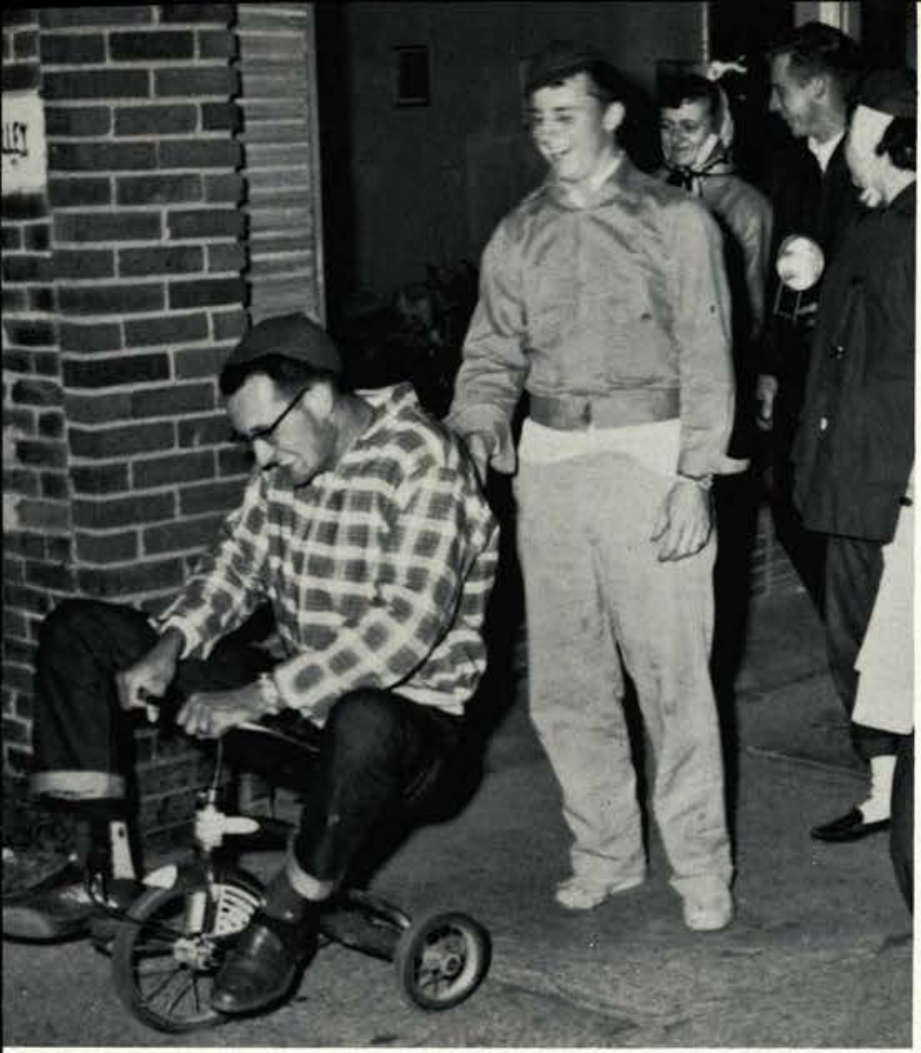




\section{Fomecaming \\ Falloween Party}

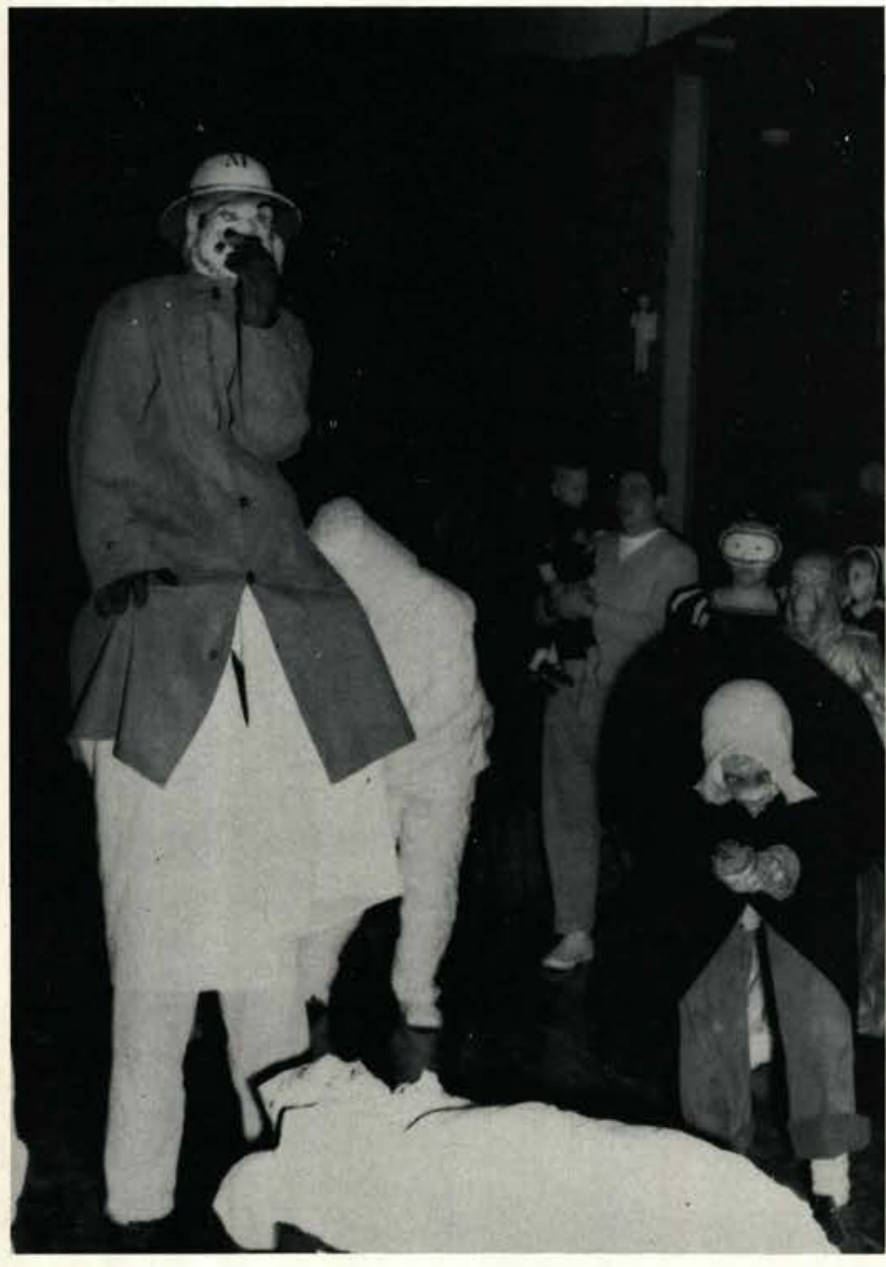

"Has anyone seen my hankie?"

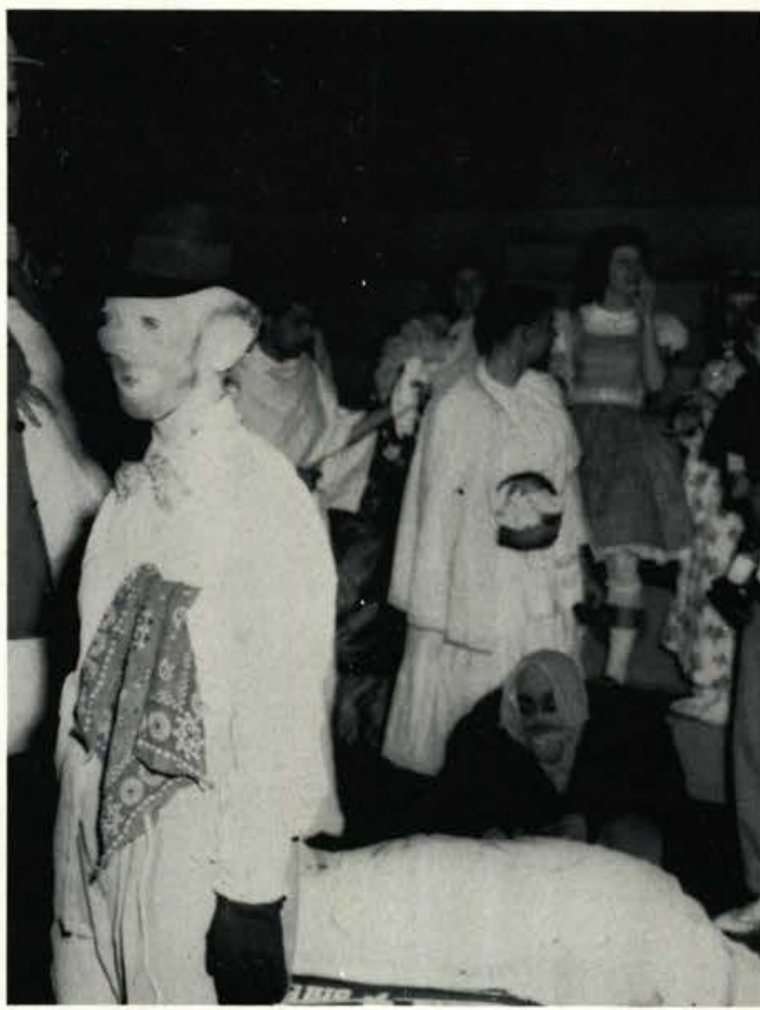

"I feel conspicuous!"

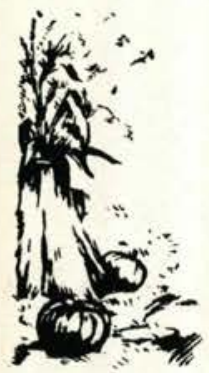

"Anything for an extra five minutes of 'sack'!"

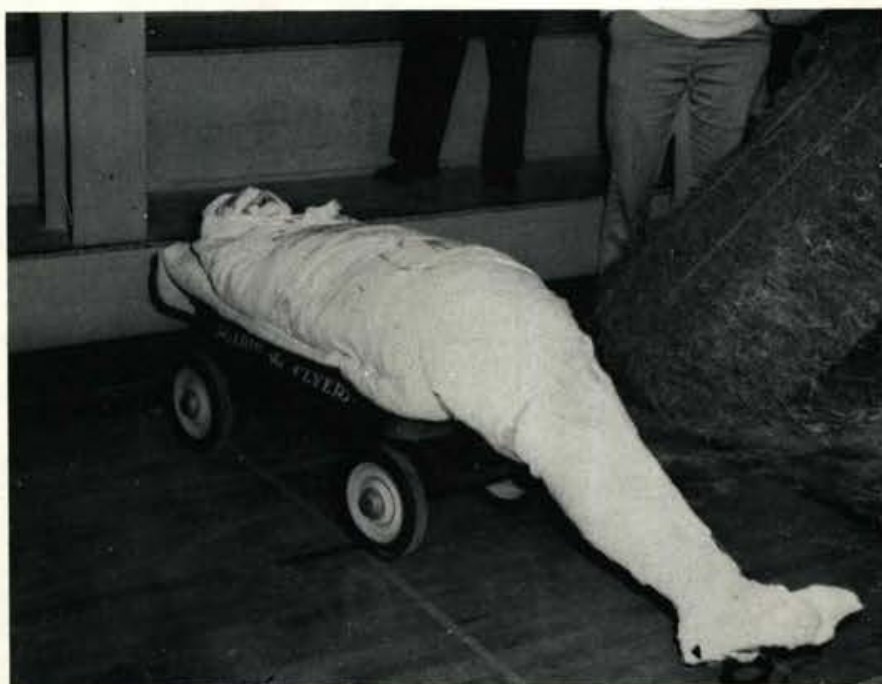




\section{Week-end}

\section{"The Night of Vamuary 16th"}
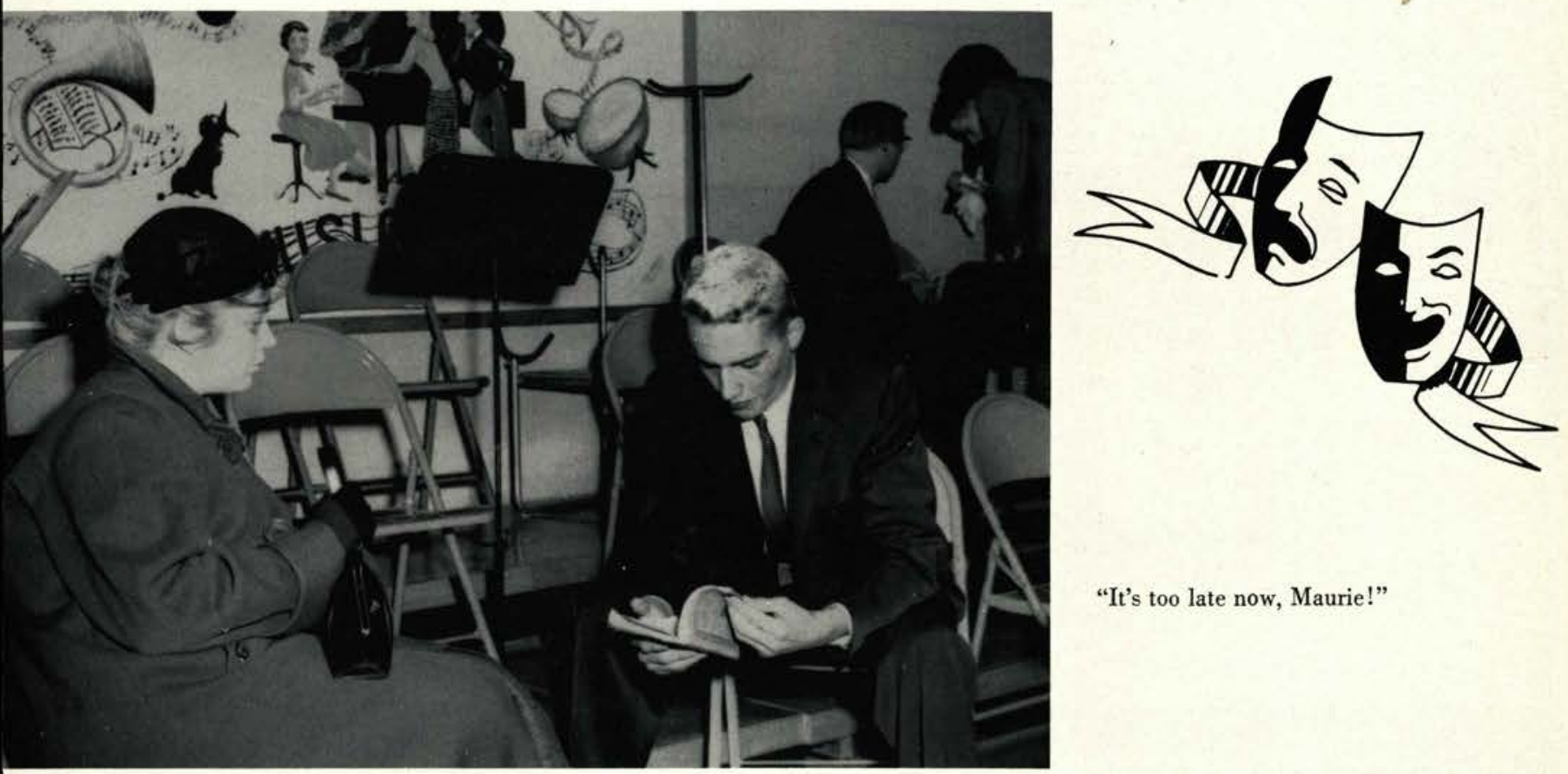

"It's too late now, Maurie!"

"Girls, girls! Don't fight!"

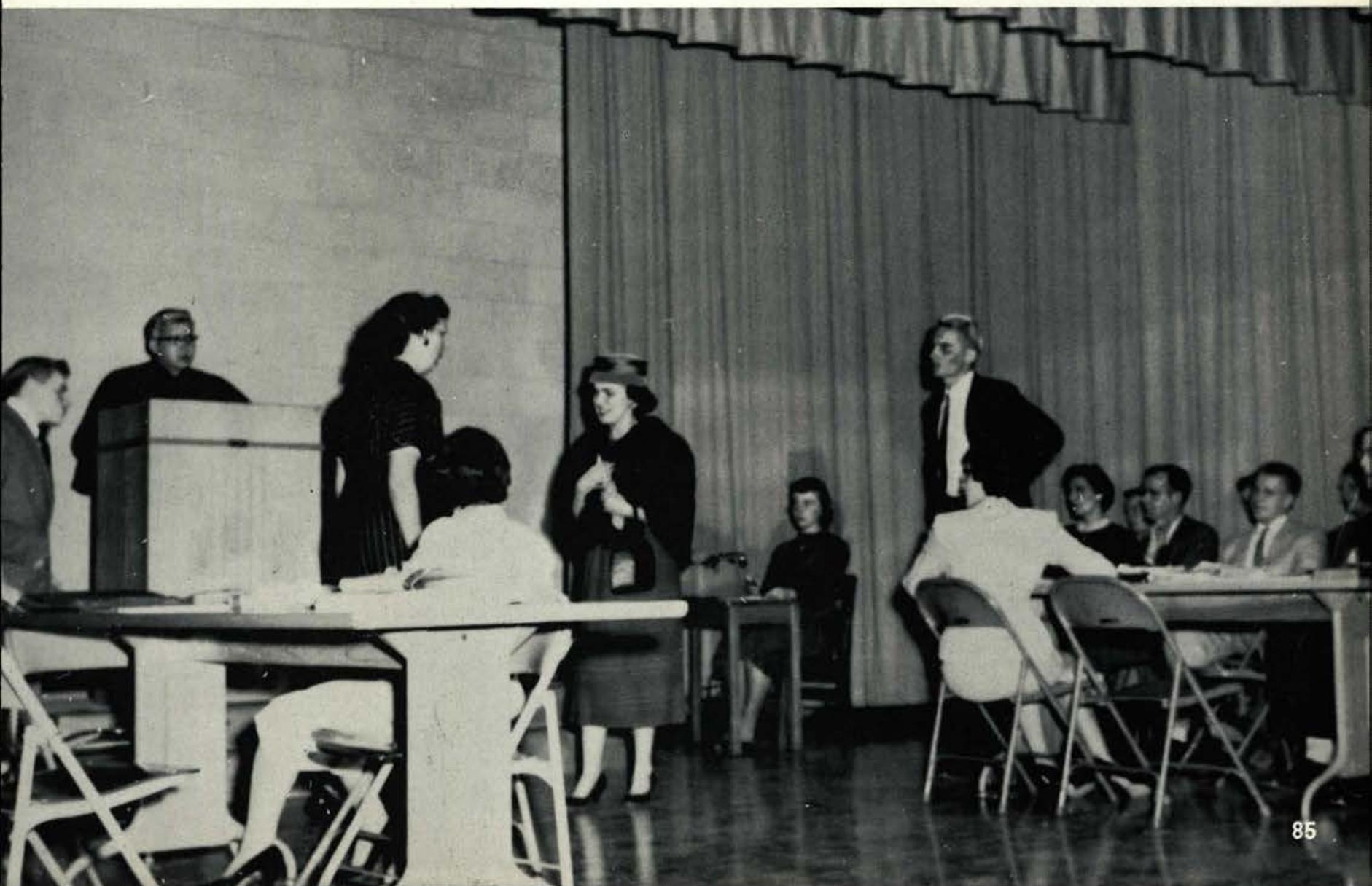




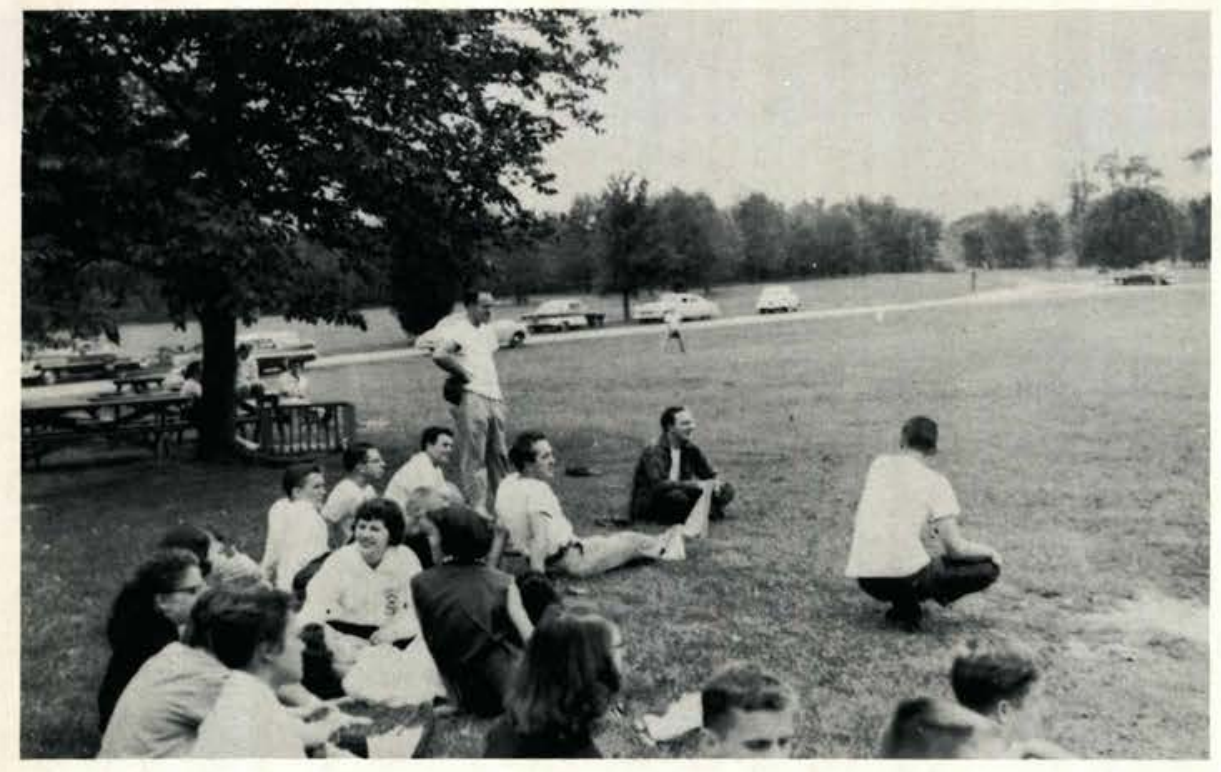

\section{Schad Dicnic}

"Calm before the storm."

\section{Sadie Haculeins' Party}

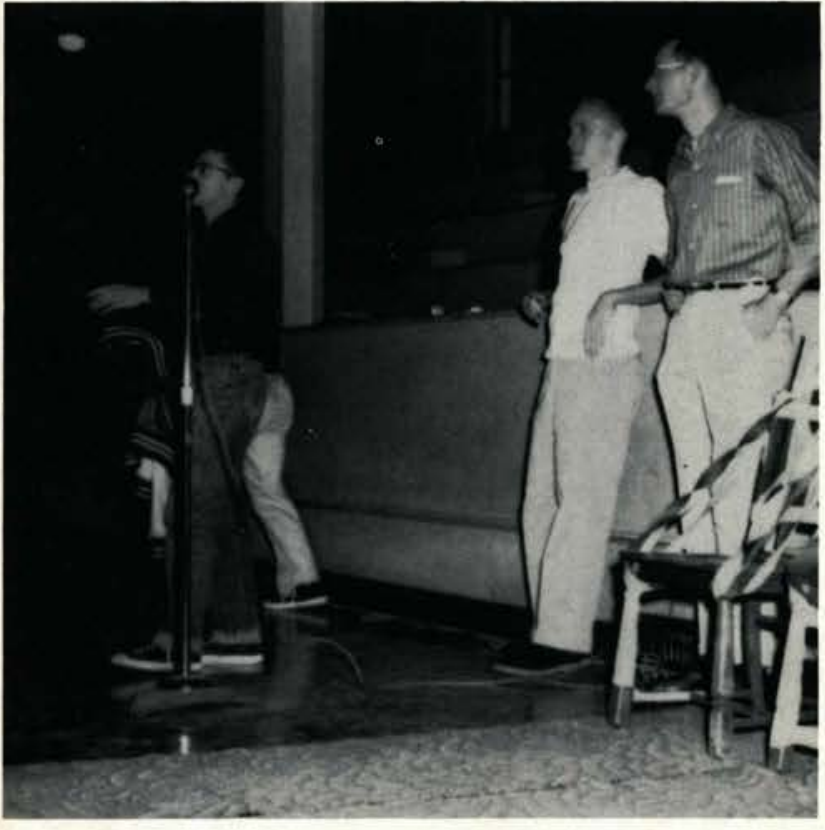

“\$2.00 and who will give me three?"

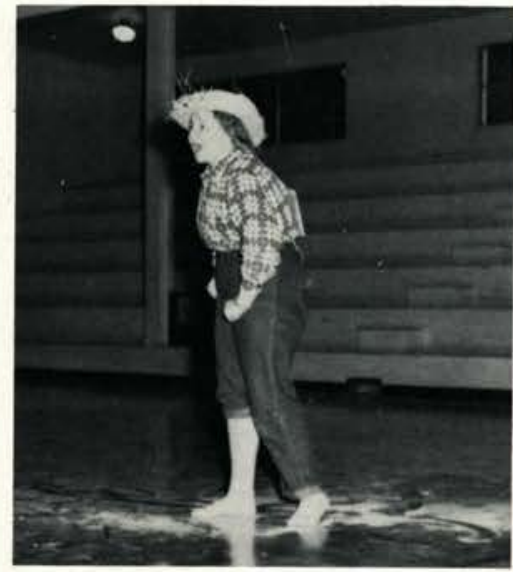

"Outa them thar hills!"

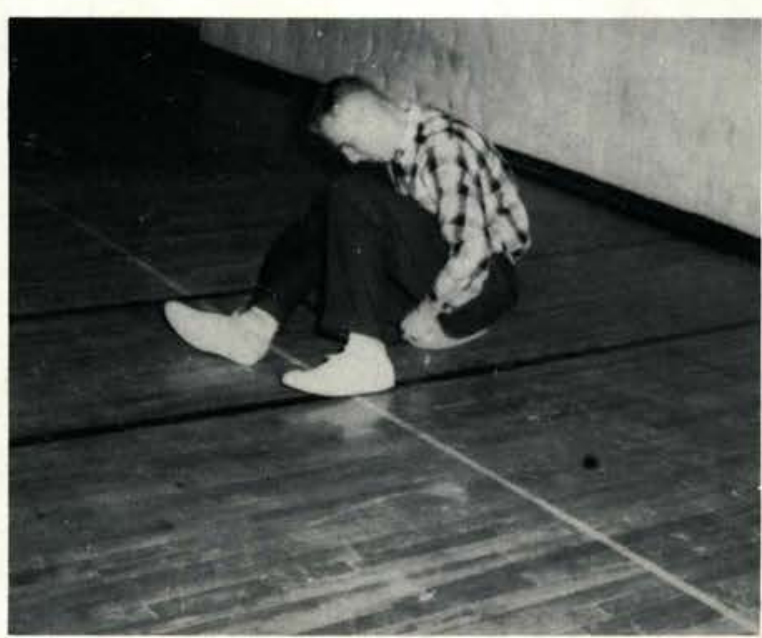

"Isn't there a formula for this too, John?" 


\section{The Sneaks by Day...}
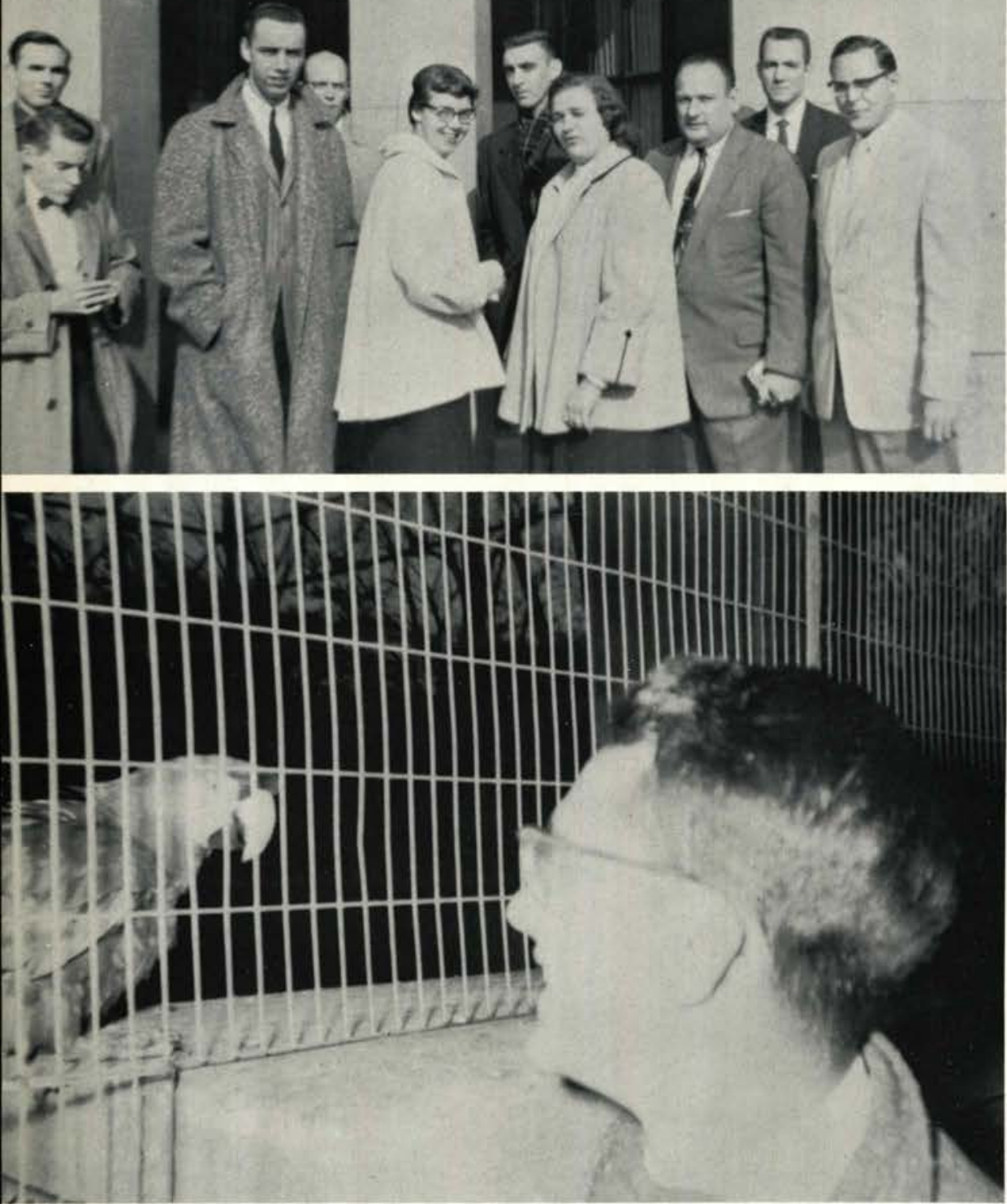


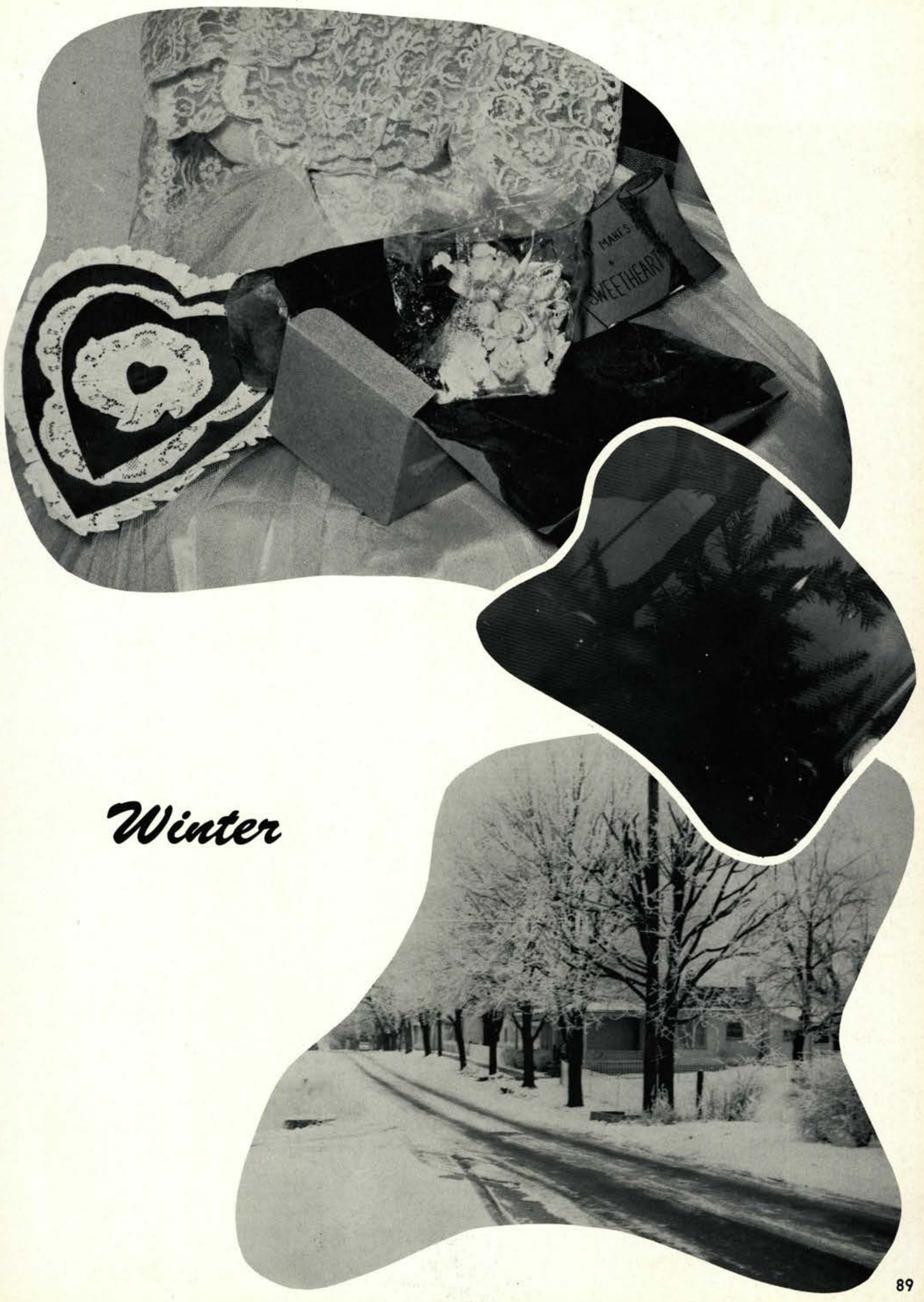




\section{The Christmas Play}

"At last, the perfect carol!"

$$
\text { (1) }
$$
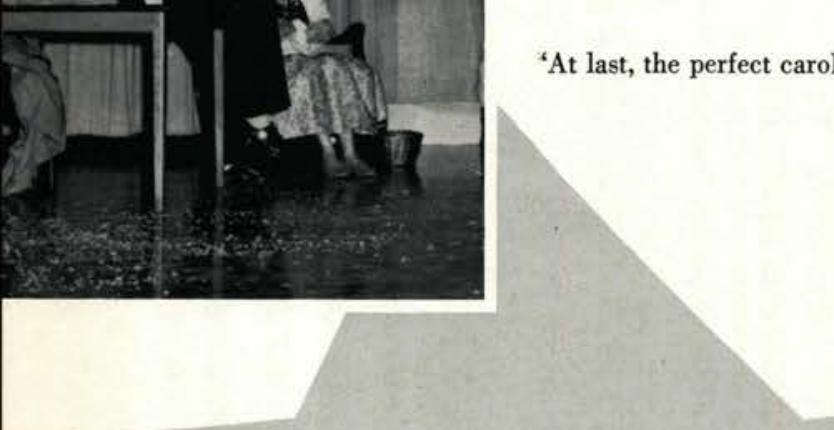

"The Perfect Card"

Pastor, come and help.

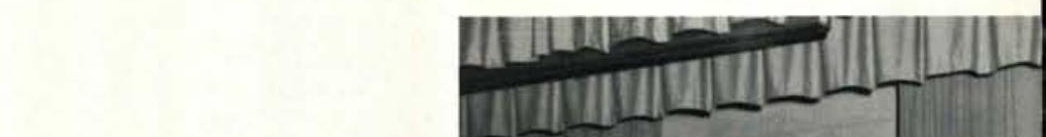

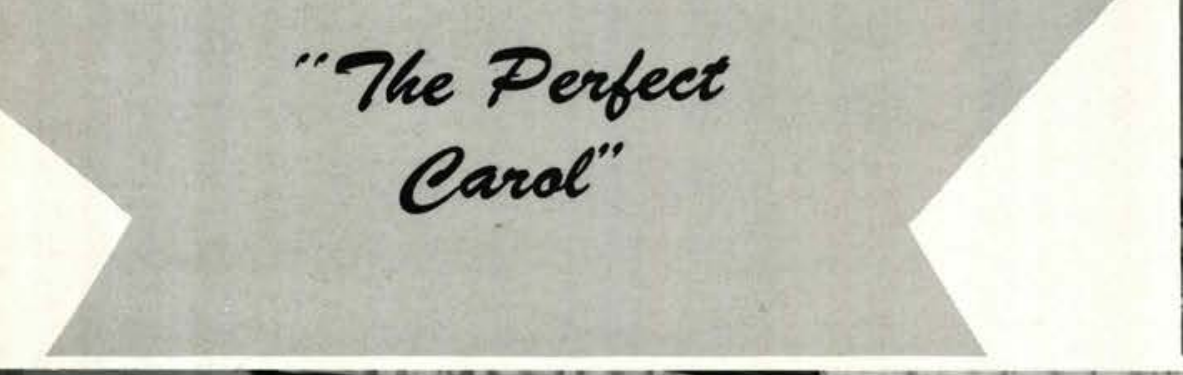

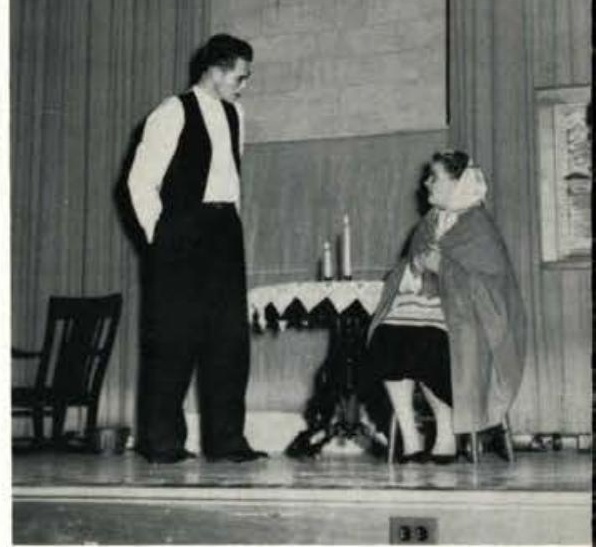

1)

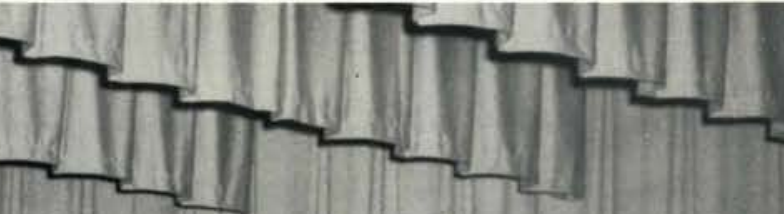

Pिलि कि

a

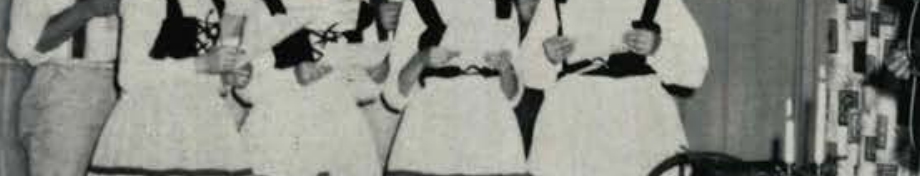

TाM 
Master of Ceremonies, MAURIE STONE

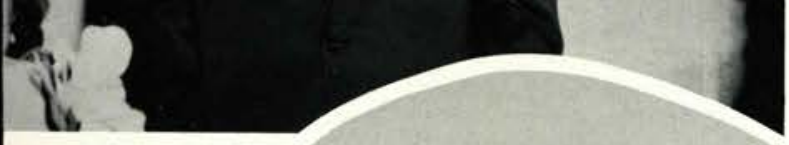

What Makes

$a$

\section{Sweetheart?}




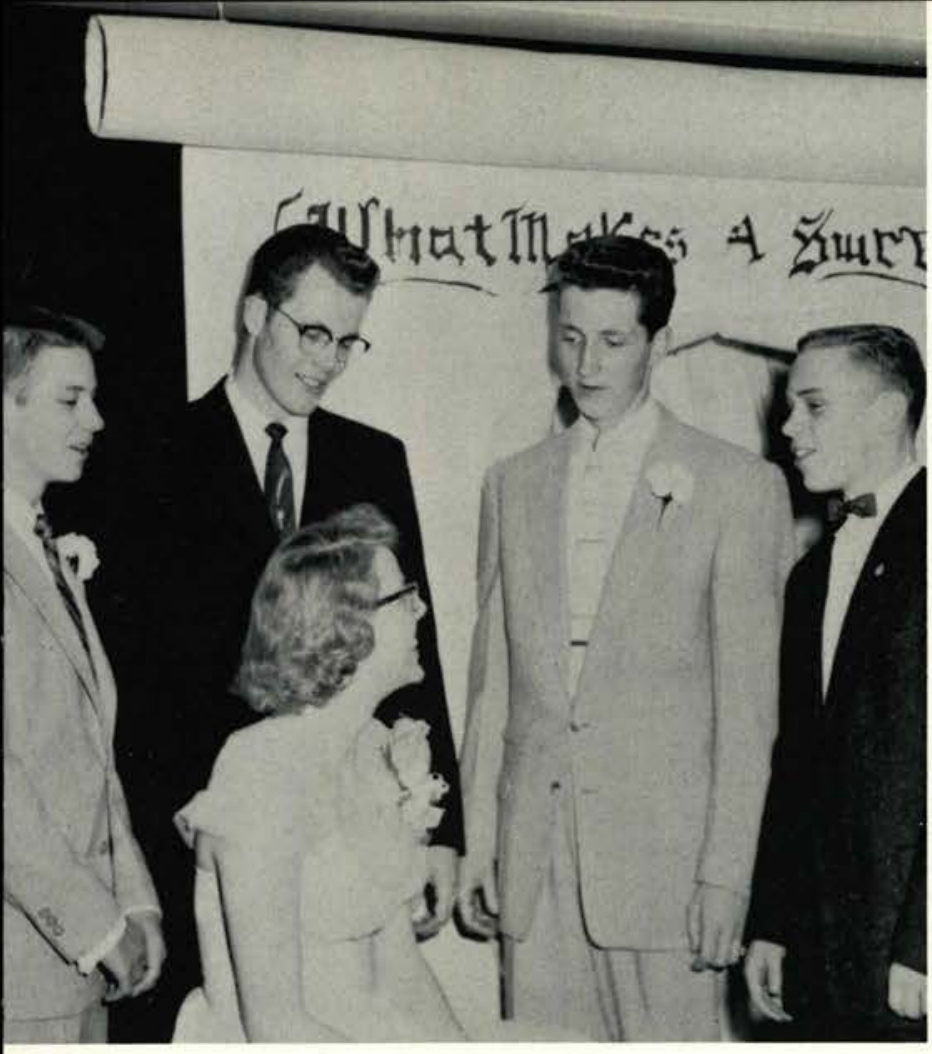

"The Sweetheart of Alpha Chi"

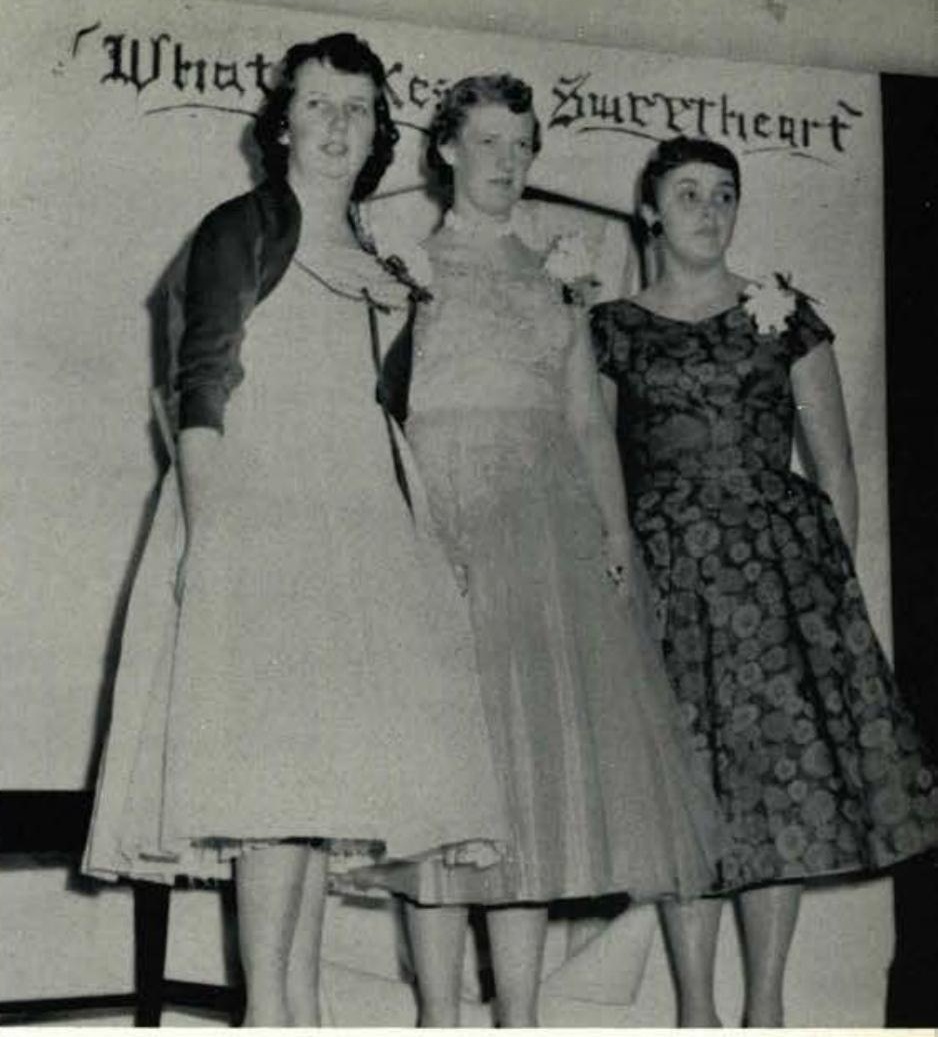

"Down by the Old Mill Stream"

\section{Banguet}

atimakes $+x^{2}$

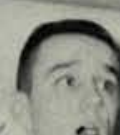

atimakes 4 zin sheart

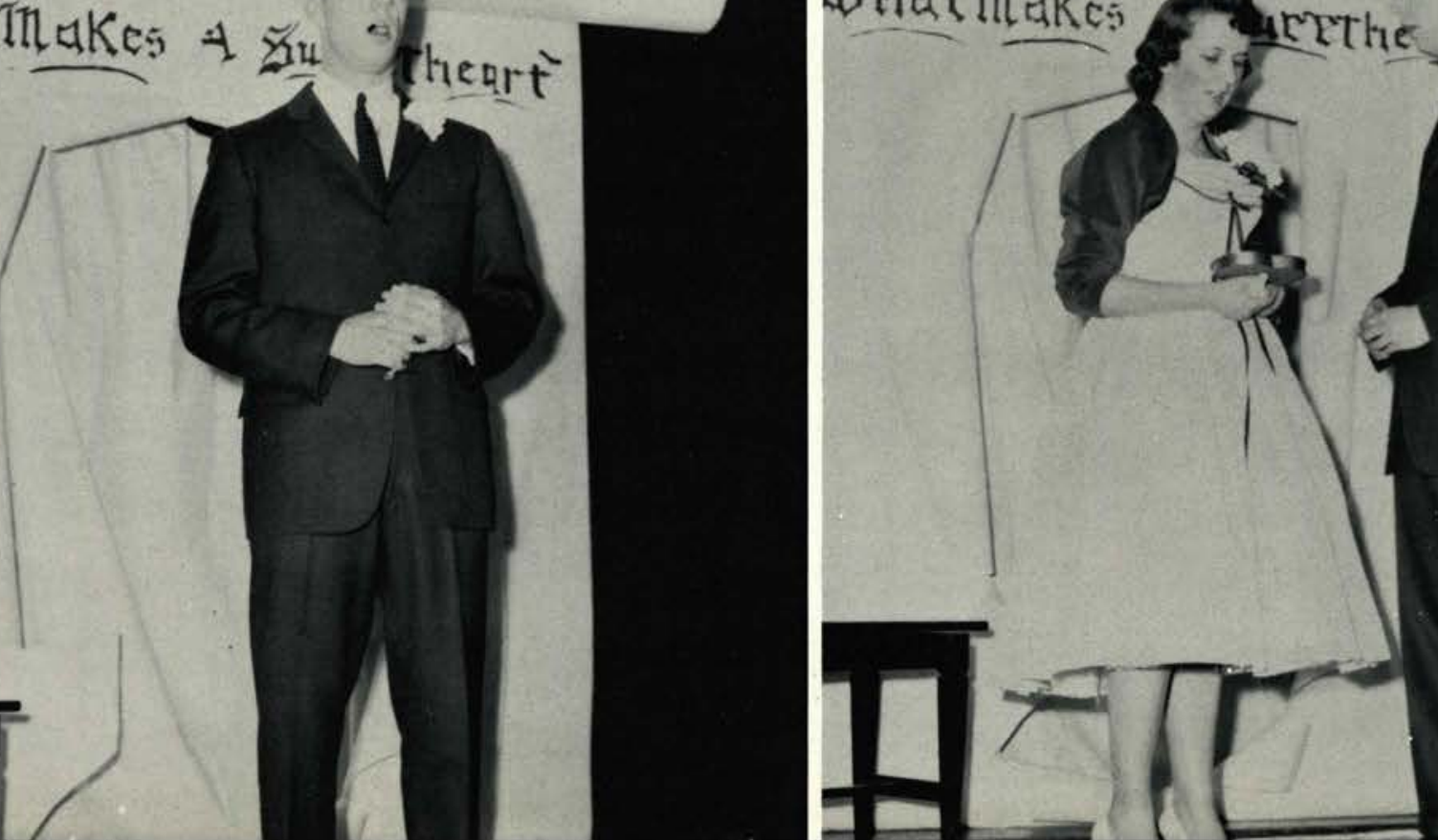




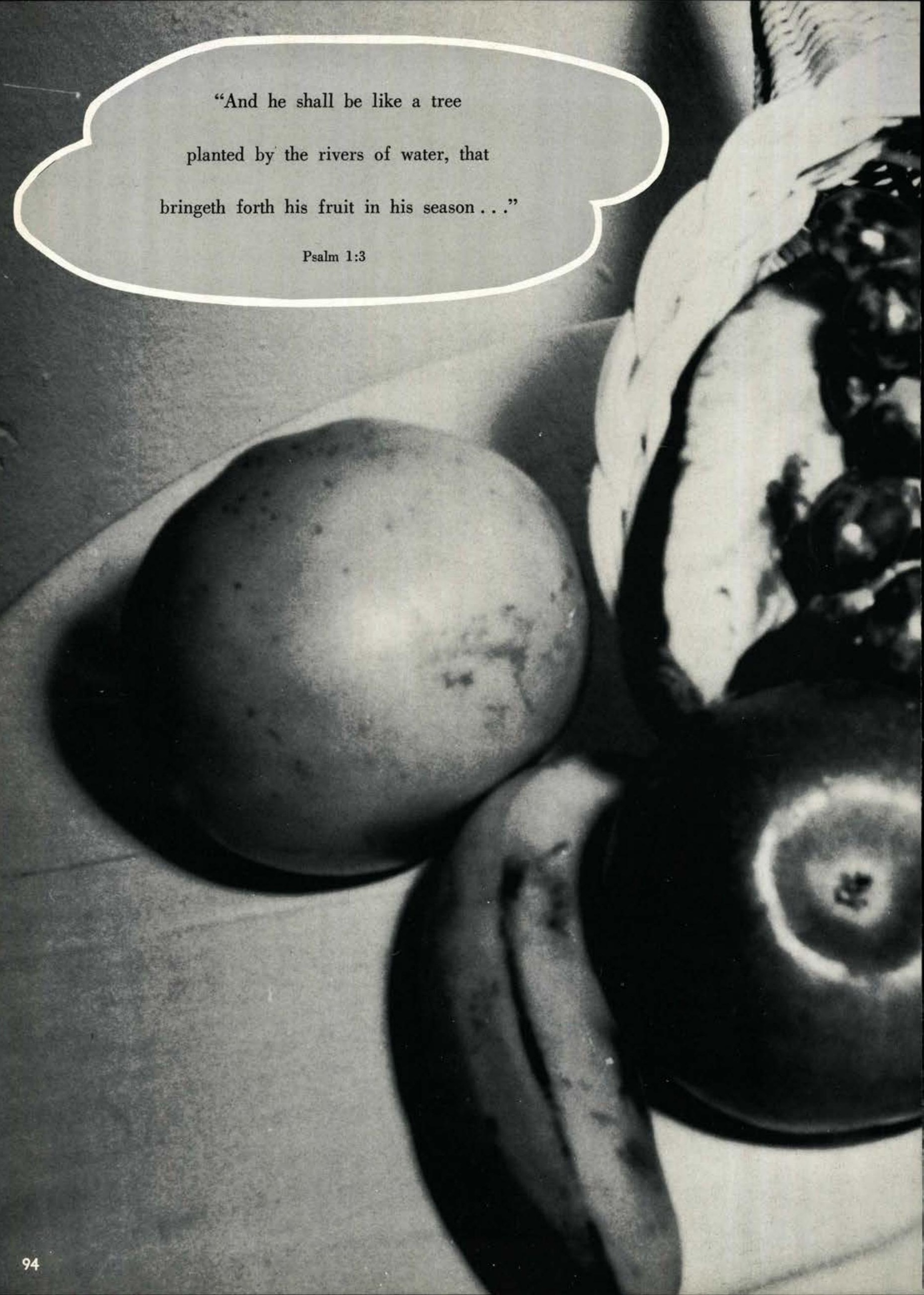




\section{Gail Ministry}

Every Sunday morning services are held in the Xenia and Springfield jails.

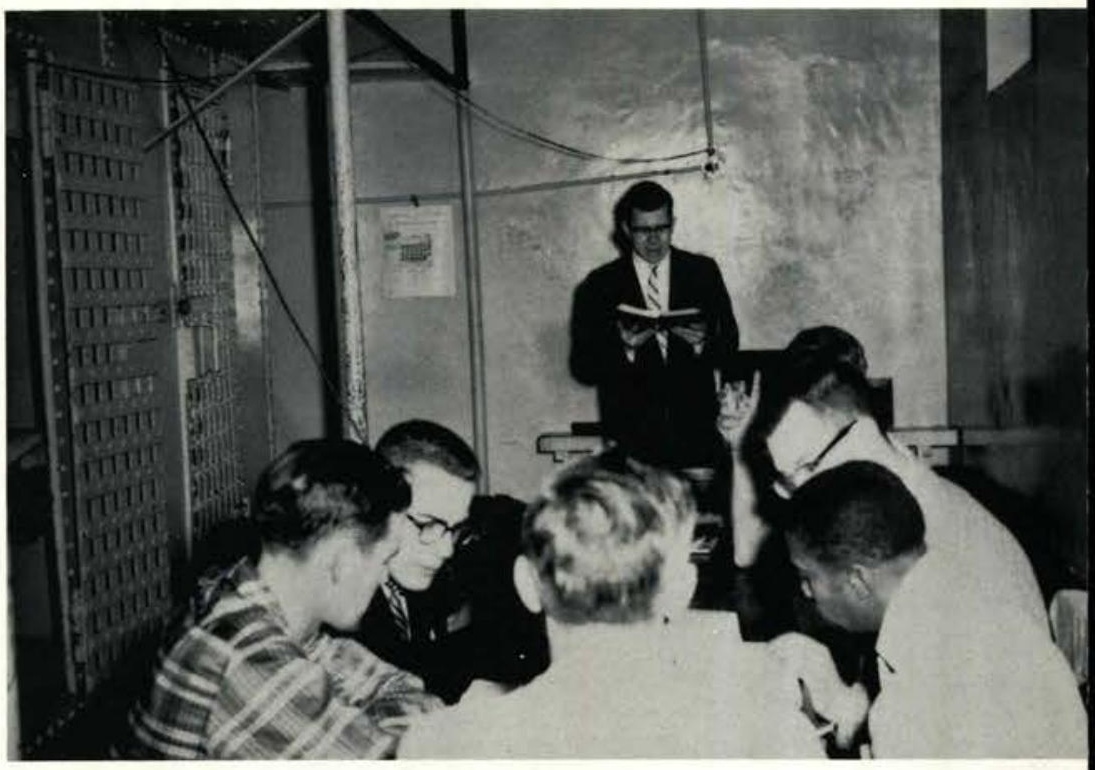

Bob Humphreys is making use of an opportunity for personal work with an inmate. Several have been saved and are being witnesses for the Lord. 


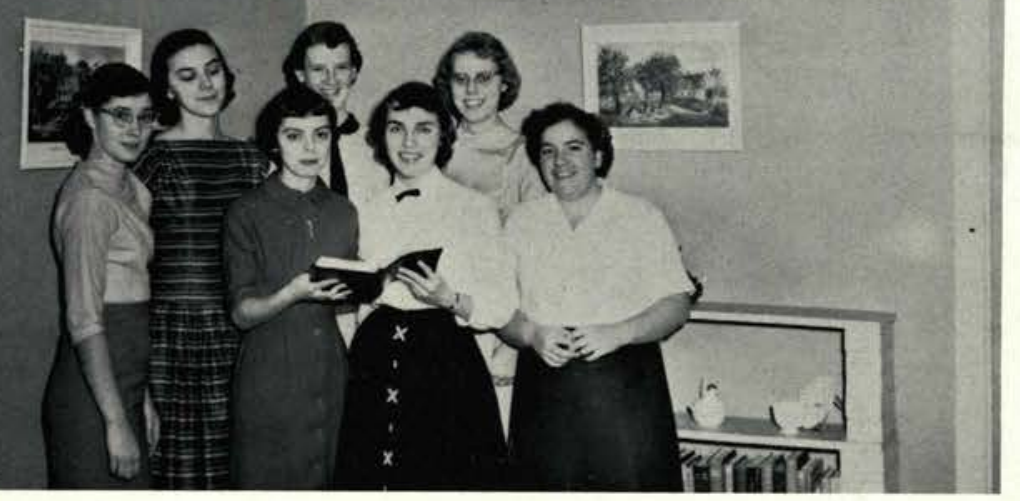

Back row: Donis Collier, Beverly VanMullen, Barbara Sherry, Carol Zoellner. Front row: Linda Johnson, Esther Weiss, Norma Nulph.
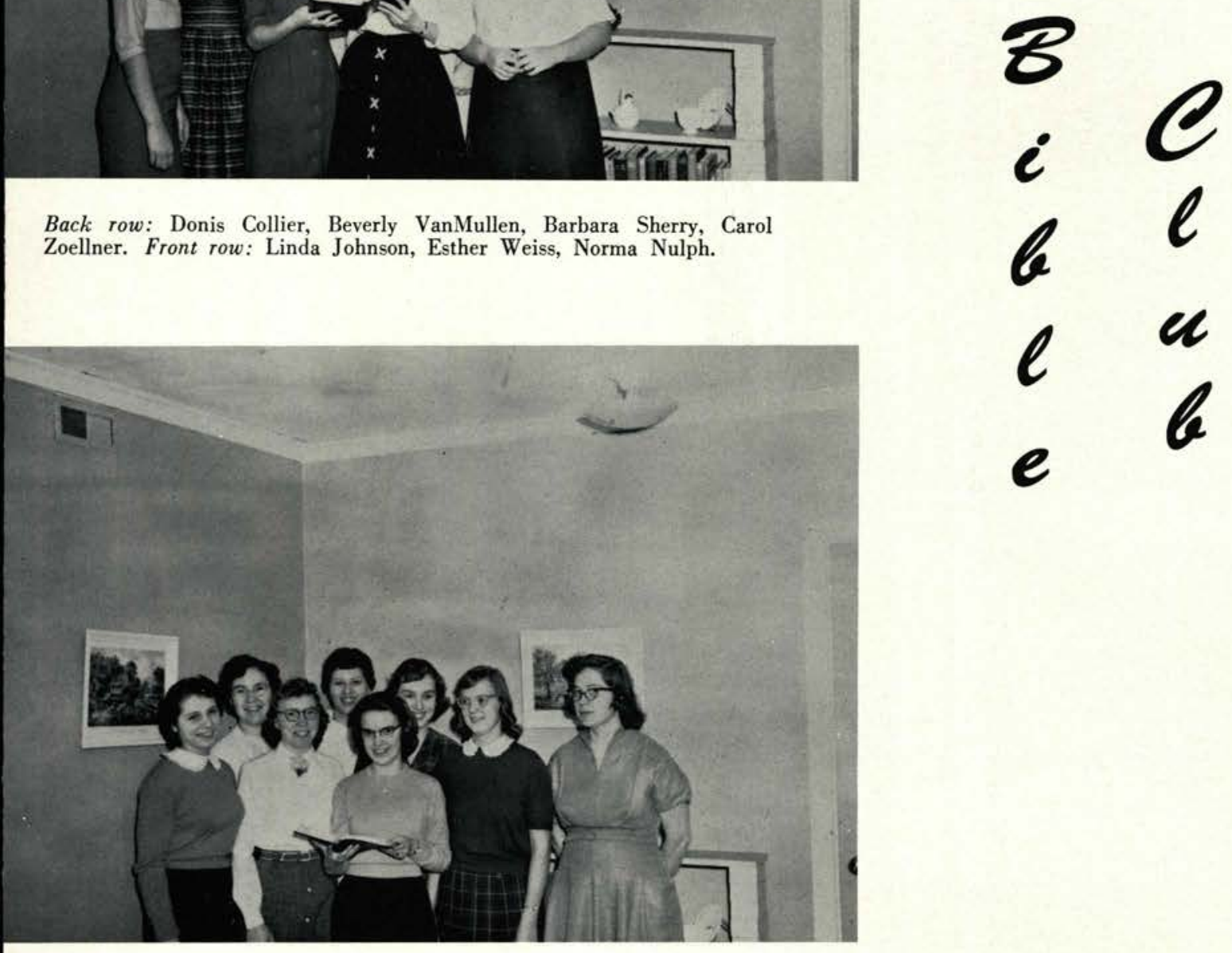

Back row: Marlene Davis, Joyce Grant, Marcia Crothers, Lucy Lyons, Carol Johnson, Neva Claypool. Front row: Phyllis Ernst, Ruth Yost.

Bible clubs provide excellent opportunities for presenting the Gospel message to boys and girls. A number of decisions have been made in the nine clubs which are held in near-by towns.

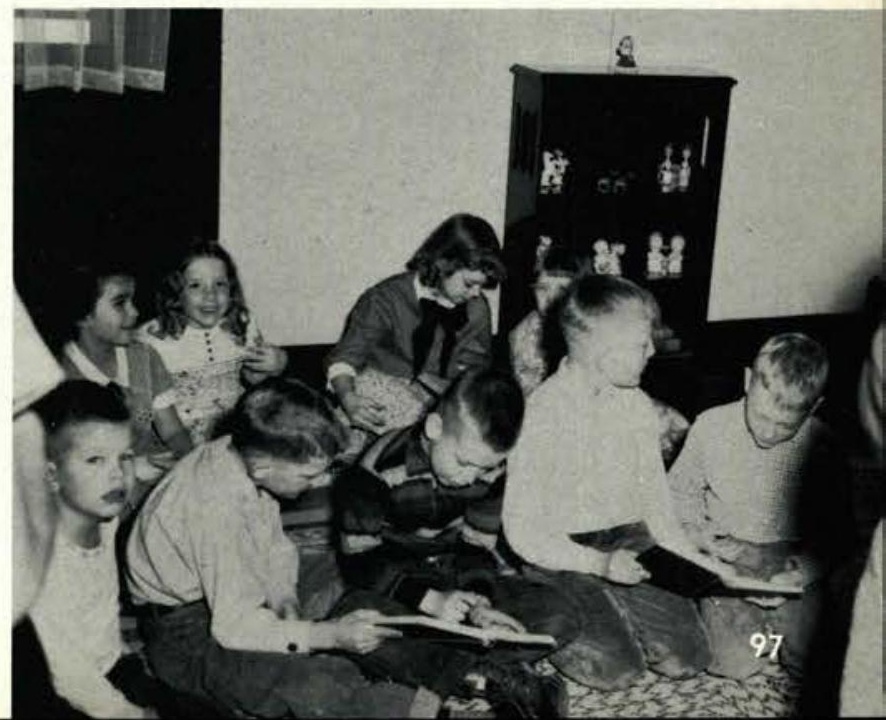




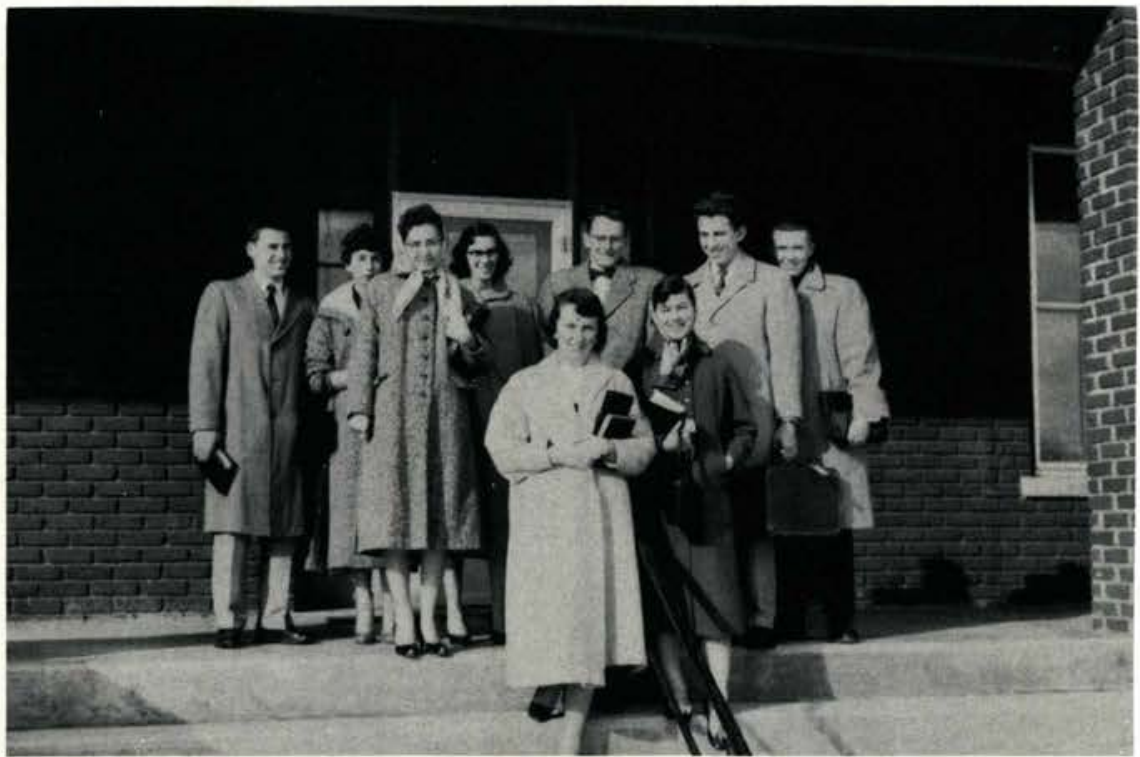

Above is a group of students visiting at the Clark County Home in Springfield.

\section{Ministry}

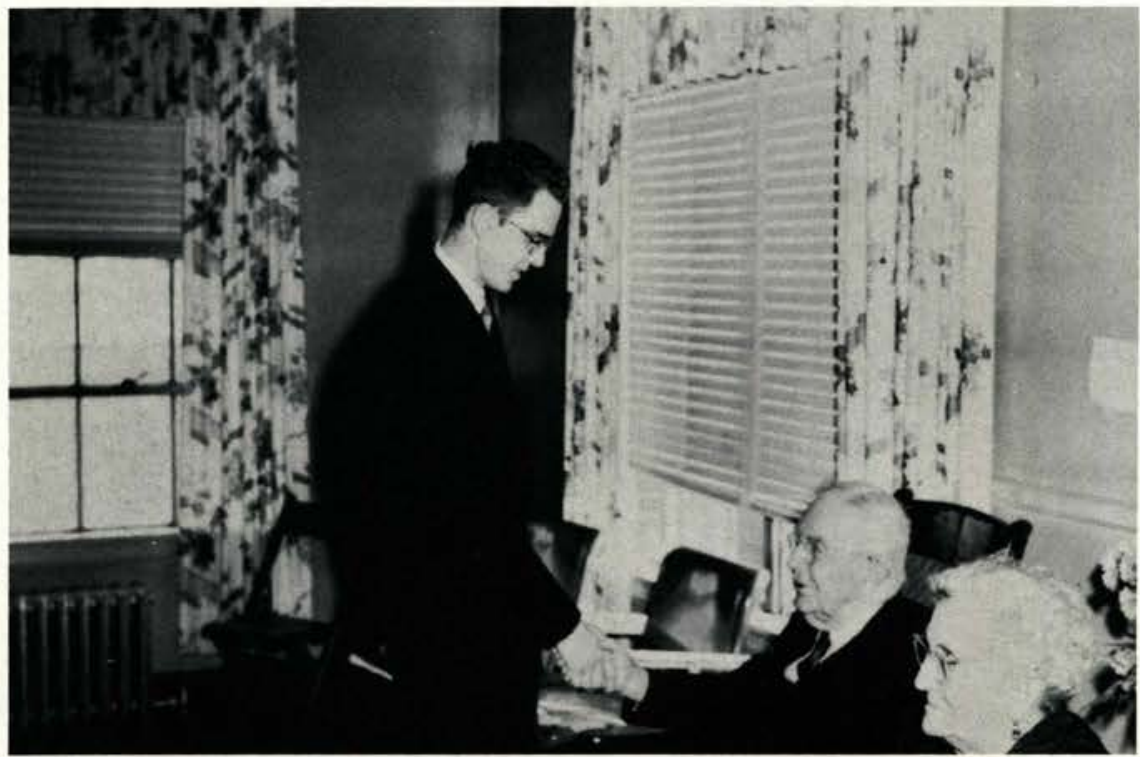

Lon Reising is greeting the folks at the Knights of Pythias Home in Springfield. Services are held each Sunday afternoon in the hospital and the annex. 


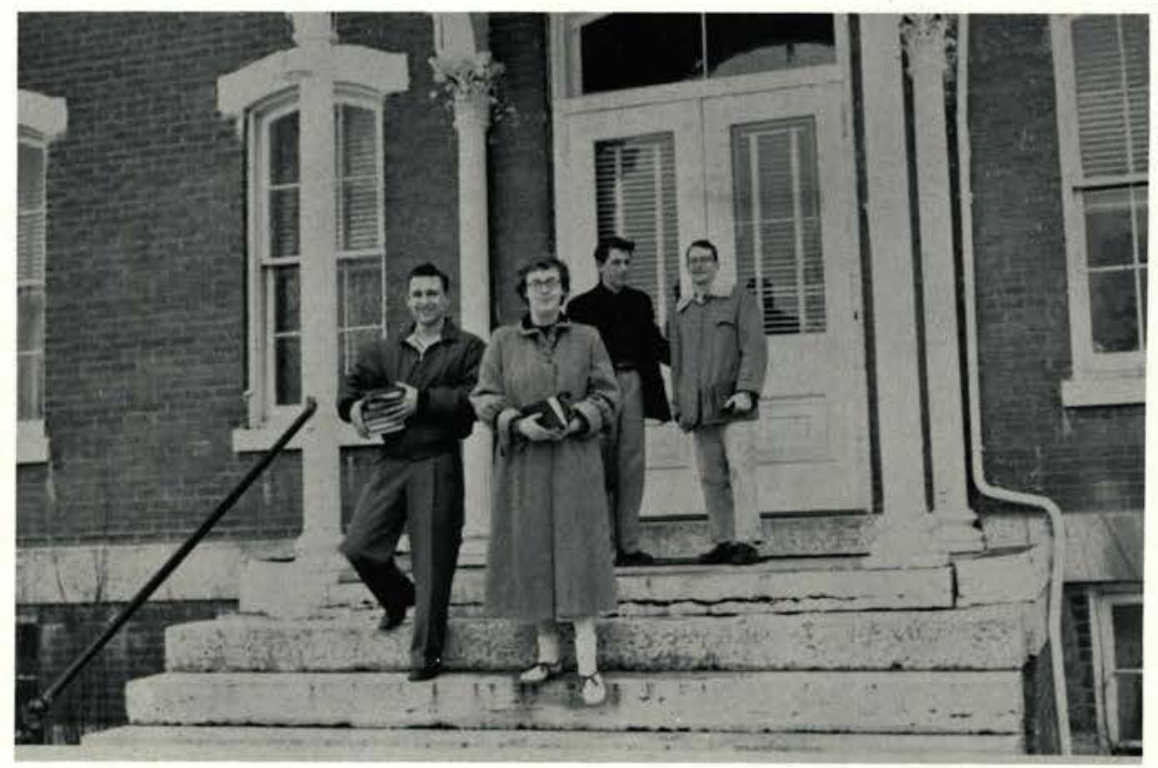

Above are several members of the group who minister at the Greene County Home in Xenia.
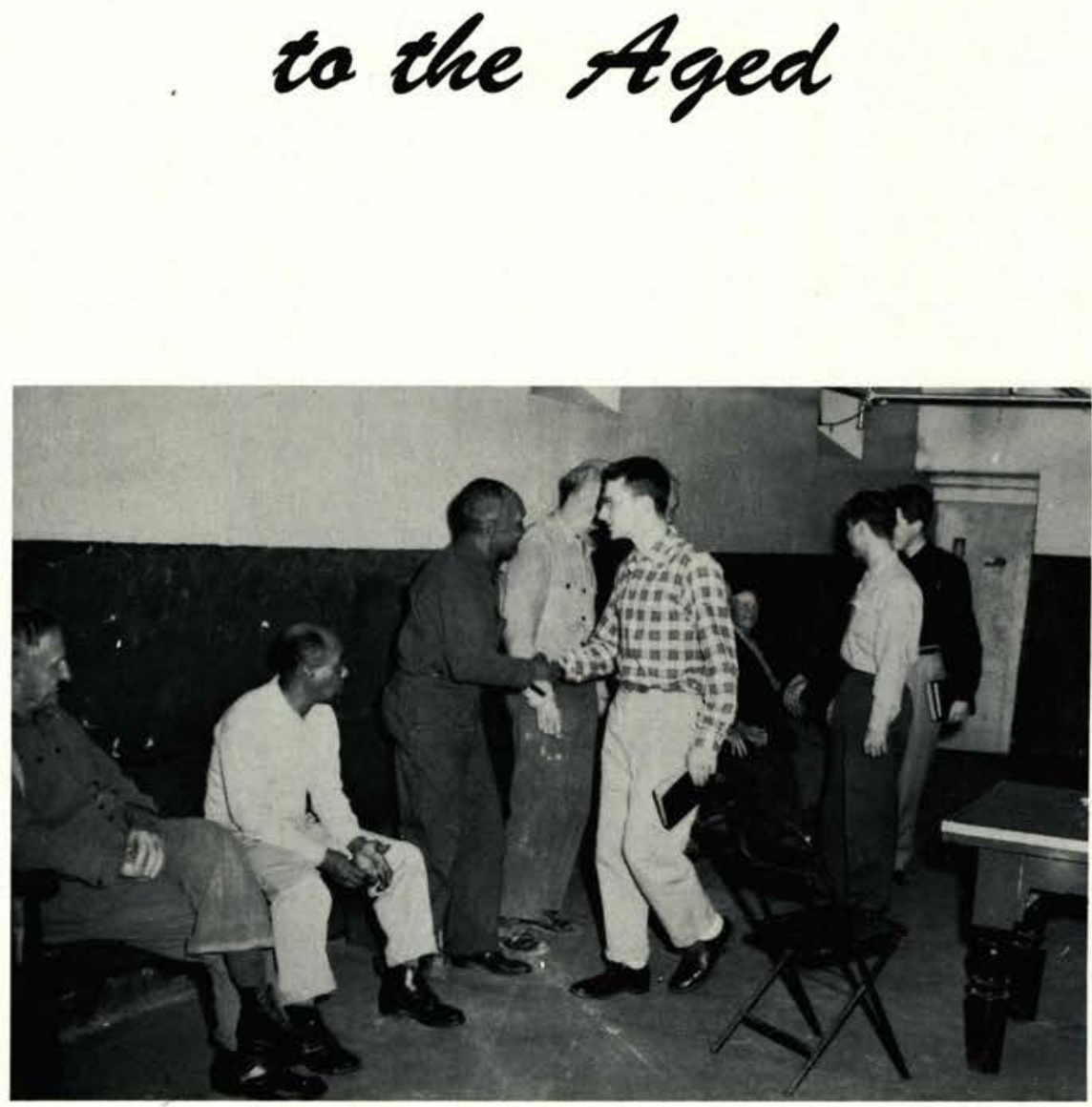

The people at the Greene County Home look forward to music, testimonies, and messages from the Word. 


\section{Veteran's Fospital}

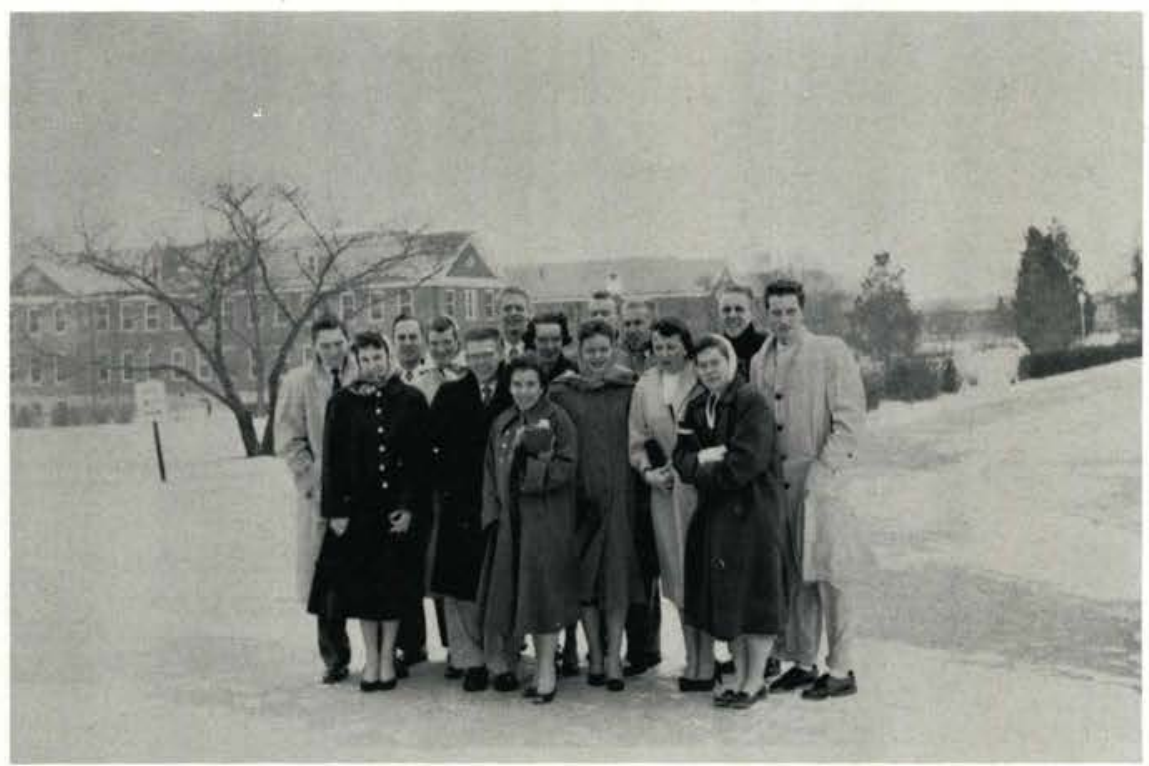

Back row: Merlin Ager, Stu.Chafee, Barbara Sherry, Paul Anderson, Rosemary Smith, Terry Goodrich, Dan Purdy, Jim Entner, Warren Woodard. Front row: Ruth Himsel, Tim Grafton, Sandy Millikin, Ruth Smelser, Grace Willetts, Neva Claypool.

A hard but fruitful ministry has been established at the mental hospital in Chillicothe where services are held each Sunday morning.

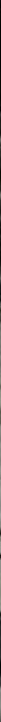




\section{BOYS' BRIGADE}

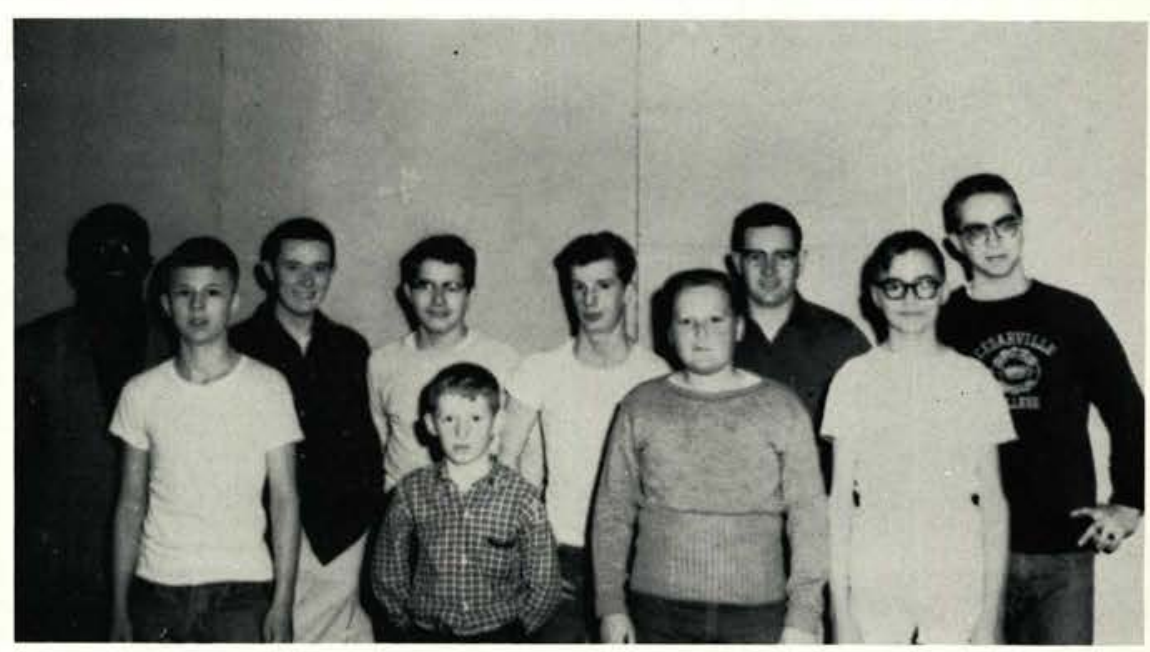

Boys' Brigade group with leaders: Bill Mason, Les Webster, Dean Mayo and Dave Matson.

The Boys' Brigade, a national organization, provides healthy activities for young teenagers. Its athletic program with a Christian emphasis helps develop well-rounded individuals.

\section{ADVERTISING}

\section{Continuing in the Same Direction}

Cedarville's graduates who are looking forward to serving the Lord in the pastorate or on the mission field will find, as have David Gardner ('56) and Roy Shelpman ('57), that the same Biblical, Baptist, Separatist and Spiritual education they have received at the undergraduate level is continued and advanced at the ...

\section{GRAND RAPIDS BAPTIST THEOLOGICAL SEMINARY \\ 8II Wealthy Street S. E. GRAND RAPIDS 6, MICHIGAN}




\section{THE HORTON FAMILY}

\author{
LAPEER, MICHIGAN
}

"But the Lord said unto Samuel, Look not on his countenance, or on the height of his stature; because I have refused him: for the Lord seeth not as man seeth; for man looketh on the outward appearance, but the Lord looketh on the heart."

I SAMUEL 16:7

\section{HARNER'S \\ CEDARVILLE MARKET}

Meats

Produce Groceries

Phone So 6-I20I

CEDARVILLE, OHIO
Compliments

\section{of \\ STOKES}

\section{MOTOR COMPANY}

South Main Street

CEDARVILLE, OHIO

Phone So 6-4021

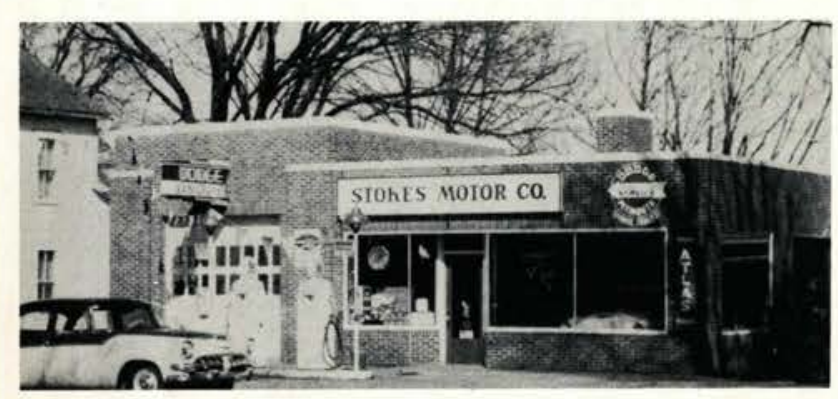

\section{FLEET WING \\ HAGLER \\ SERVICE STATION}

Phone So 6.0110

CEDARVILLE, OHIO 


\title{
Best Wishes HALE DRUGS
}

\author{
Your Walgreen Agency \\ Friendly Personalized Service \\ PRESCRIPTION SPECIALIST
}

Telephone 2-3113

5692 MAIN STREET SYLVANIA, OHIO

\section{BEATY \& FINNEY}

\section{SHELL SERVICE STATION}

Cedarville, Ohio

Phone So. 6-3711

Tires

Car Wash
Shellubrication

Batteries

\section{CHAPLIN CLEANERS}

Laundry Service

Cleaning

Shoe Repair

Pressing

CEDARVILLE, OHIO
The Scripture states, "Know ye not that ye are the temple of God." Are you proud and happy that you are offering $\mathrm{Him}$ a sick body to dwoll in? Do you think he is? If you neglect your body's health and life, your soul will take flight, what then will your college education avail you and others? Don't suffer and die in despair - get a JUICEX. Try drinking God's natural goodness in liquid form. It helped others, it may help you.

Supplement your meals with the 16 basic organic minerals, vitamins and enzymes by drinking raw fresh vegetable juices extracted in your own home. These are full of health sustaining nutrients, whereas cooked foods refined, chemicalized, bleached, embalmed and synthetics are dead foods. Don't expect to feed on such fillers and feel like a million.

The FAMOUS JUICEX is a small home electric vegetable juice extractor. So simple that a child can operate it. You can't eat a dozen raw carrots or a few pounds of spinach in one sitting, but you can drink the goodness extracted therefrom in one sitting.

A healthy long lived graduate can do more for the purpose for which he went to Cedarville College.

\section{DRACHENBERG PRODUCTS MFG. CO. 23600 Harper Avenue St. Clair Shores, Michigan}




\section{MIAMI DEPOSIT BANK}

Complete Facilities

Ready to Serve You YELLOW SPRINGS, OHIO and

CEDARVILLE, OHIO

Member of the

FEDERAL DEPOSIT

INSURANCE CORPORATION

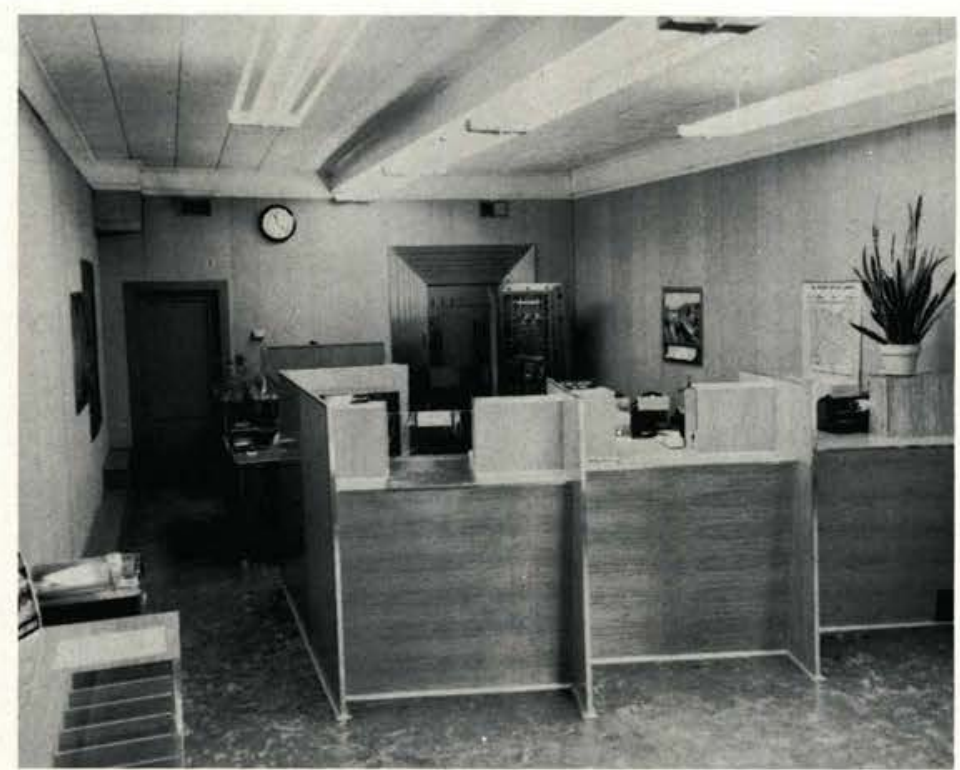

WELLL DRILLING CONTRACTOR

Sizes 3 " to 12"

Turbine and Jet

Pumps

Complete Water

Systems

Free Estimates

F.H.A. Terms

HOWARD C. TAYLOR

I335 Peppermill Rd.

\section{RANDALL \\ \& \\ STORMONT}

Feed and

Supply

Phone So. 6-1031

CEDARVILLE

$\mathrm{OHIO}$

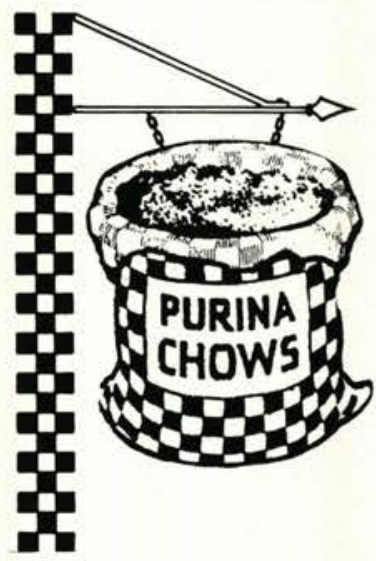

Studebaker

Packard

WHATTOFF MOTOR CO.

AMES, IOWA 


\section{Compliments \\ of}

\section{MORRIS BEAN \\ AND COMPANY}

YELLOW SPRINGS, OHIO

CEDARVILLE, OHIO

\section{JAMES CUT-RATE}

The Rexall Store

Phone So. 6-177|

CEDARVILLE, OHIO
CEDARVILLE COLLEGE BOOK STORE

Student Supply

Center

\section{BAPTIST MID-MISSIONS \\ 1120 Chester Avenue CLEVELAND 14, OHIO}

Baptist Mid-Missions is an independent fundamental Baptist missionary council with 600 missionaries serving in 21 foreign countries, 20 states in the United States as well as Canada, Alaska and Hawaii. As a result of this endeavor there are over $\mathbf{2 5 0 , 0 0 0}$ believers trusting the Lord. Are you concerned that thousands are still waiting to hear the Gospel?

"THE FIELD IS THE WORLD" 
Congratulations to the Graduates of 1958

\title{
CALVARY BAPTIST CHURCH
}

\author{
Crawfordsville, Indiana \\ PAUL M. HUBBLE, Pastor \\ We Encourage the 1958 Graduating Class \\ to \\ "Contend Earnestly for the Faith \\ Once Delivered Unto the Saints"
}

Congratulations and the Lord's Blessing

to the 1958 Graduates

MEMORIAL BAPTIST CHURCH

20I South Main Street

VERONA, WISCONSIN

"The Church in the Heart of the Village

With the Village at Heart"

WILLIAM F. HARRIS, Pastor

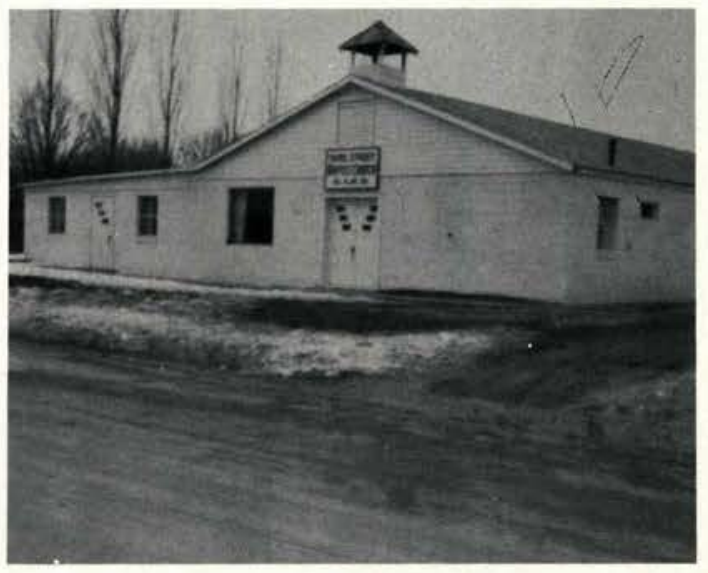

\author{
THIRD STREET \\ BAPTIST CHURCH \\ EAST MOLINE, ILL. \\ FRED G. CROWN \\ Pastor \\ CLIFFORD DUDLEY \\ S. S. Supt.
}

A Small Church with a Spiritual Program partially Supporting Four Missionaries

G.A.R.B. Affiliation

Record Attendance in Sunday School 234

Two Young People in Baptist Colleges

Installation of Pews in Progress

"We Preach Christ Crucified, Risen, Glorified, Coming Again" 


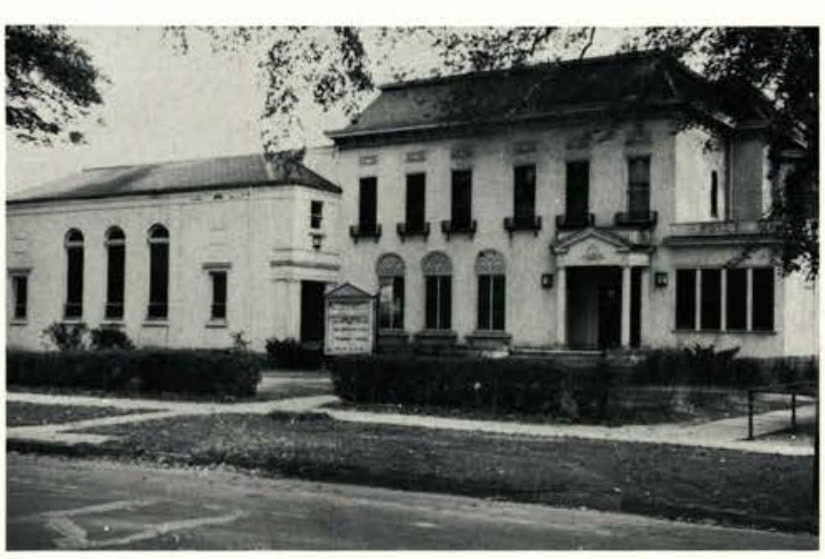

\author{
EMMANUEL \\ BAPTIST CHURCH \\ Grand at Waite, TOLEDO, OHIO \\ Pastor K. L. ANDRUS \\ Asst. Pastor. \\ SUNDAY SERVICES \\ 10:00 Sunday School \\ 11:00 Morning Worship \\ 6:30 Youth Groups \\ 7:30 Evening Service \\ Midweek Prayer Hour-Thursday 7:30 \\ On the Air Every Sunday Morning \\ WTOL 9:15 to $9: 30$ \\ WSPD $\quad 11: 15$ to $11: 30$
}

\title{
COSPERVILLE BAPTIST CHURCH
}

\author{
WAWAKA, INDIADA \\ REV. LEONARD UHRICH, Pastar
}

"FOREVER, O LORD, THY WORD IS SETTLED IN HEAVEN."

Psalm 119:89

\section{BEREA BAPTIST CHURCH}

\author{
250 West Street \\ Berea, Ohio \\ SERVICES
}

Bible School

9:30 A.M.

Morning Worship 10:45 A.M.

Bible Training Union 6:45 P.M.

Evening Gospel Service 7:45 P.M.

Wednesday Prayer Meeting

7:45 P.M.

"That in All Things He Might Have the Pre-eminence." EARL V. WILLETTS, Pastor 


\section{When in Florida, Attend the EMMANUEL BAPTIST CHURCH \\ DAVID T. JORDAN, Pastor \\ 4I2I Garden Ave. \\ West Palm Beach \\ Independent-Pre-Millenial \\ Missionary-Evangelistic}

\section{MIDVIEW BAPTIST CHURCH}

\section{NORTH EATON, OHIO}

Corner of Route 82 and 76

REV. FRANK ODOR, Pastor

"Therefore, my beloved brethren, be ye stedfast, unmovable, always • abounding in the work of the Lord, forasmuch as ye know that your labour is not in vain in the Lord."

I Cor. 15:58

\section{FIRST BAPTIST CHURCH}

LA GRANGE, OHIO

TESTIFYING OF GOD'S GRACE

EDWARD C. HEMICK

Pastor 


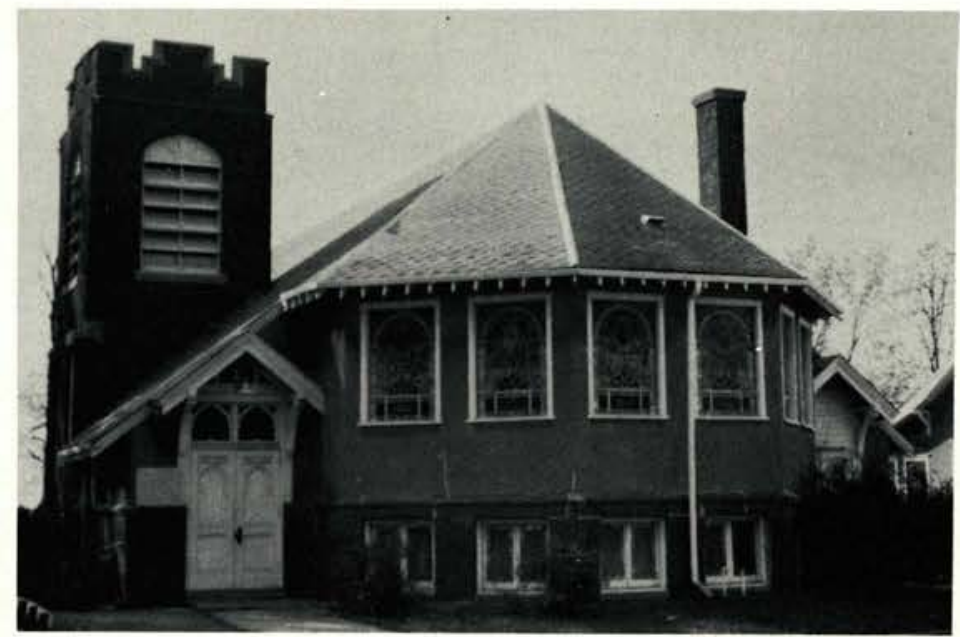

Congratulations

From the

FIRST BAPTIST

$\mathrm{CHURCH}$

of

RUSSELL, IOWA

\section{EAST SIDE BAPTIST CHURCH}

330 lowa Avenue

LORAIN, OHIO

VERNE L. DUNHAM, Minister

"A Friendly Family Fellowship"

Regular Services Held Each Lord's Day

Congratulations, Class of 1958

\section{GRACE BAPTIST CHURCH}

\section{OF CEDARVILLE}

HARRY E. COLE, Pastor

"Your Home Church Away From Home"

WE PREACH CHRIST CRUCIFIED—RISEN_COMING AGAIN 


\section{Greetings and Best Wishes \\ From the \\ FIRST BAPTIST CHURCH \\ OF PARMA, OHIO}

5994 Ridge Road, Parma 29, Ohio Tel. Tuxedo 4-2990

ELLIOTT HORTON, Pastor-45I4 Pershing Ave., Parma 34, Ohio

Sunday School 10:00 A.M.

Bible Preaching II:00 A.M. 7:30 P.M.

Mid-Week Service 7:30 P.M. Wed.

"A Christ Centered Church With a Bible Teaching Ministry"

Congratulations-Best Wishes

From the

FIRST BAPTIST CHURCH

120 Main Street Hamburg, New York

"A Friendly Church in a Friendly Village"

May God's Richest and Best Be Your Portion in

Your Training and Future Service for Him.

ALVIN G. ROSS, Pastor

MEL W. NEGLEY, Associate

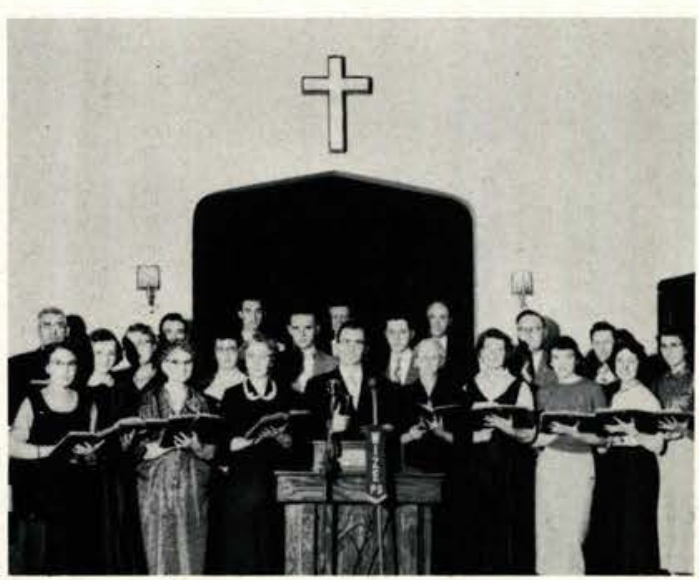

\section{BLESSED HOPE BAPTIST}

Selma Road and York Street SPRINGFIELD, OHIO

Sunday School

9:30 A.M.

Morning Worship 10:45 A.M.

Young Peoples Meeting 6:15 P.M.

Evangelistic Service 7:15 P.M.

Wednesday Prayer Hour 7:00 P.M.

Looking for. That Blessed Hope Titus 2:13

Sunday Radio Broadcast on WIZE

From 7:30-8:00 P.M.

GLENN GREENWOOD, Pastor 


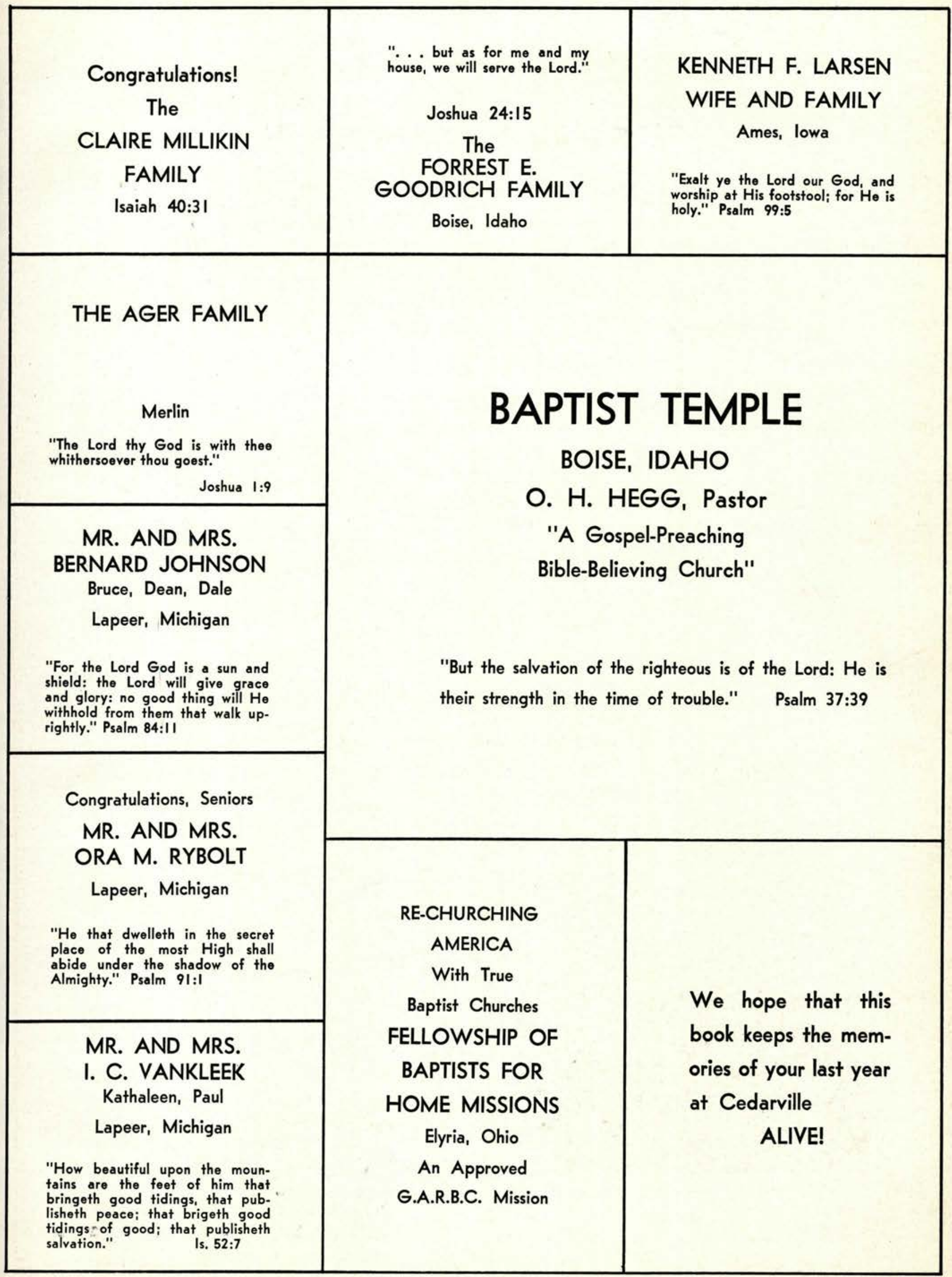




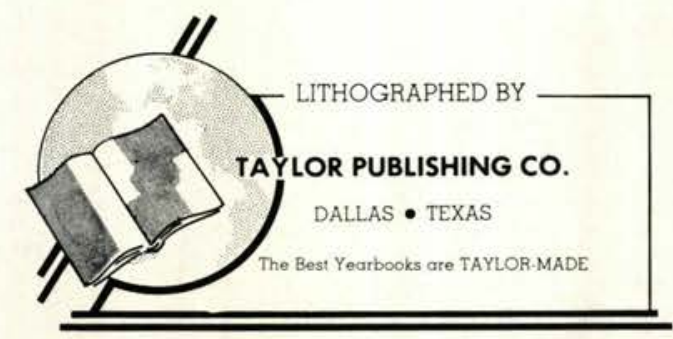





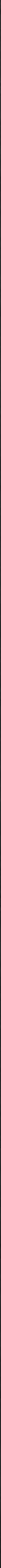

